\title{
COMPOSITION AND PHYSICAL PROPERTIES OF AQUEOUS EXTRACTS FROM PORTLAND CEMENT CLINKER PASTES CONTAINING ADDED MATERIALS
}

\author{
By George L. Kalousek, C. H. Jumper, and J. J. Tregoning
}

\section{ABSTRACT}

Mixtures of 12 commercial portland cement clinkers and water were filtered at 7 minutes and at 2 hours after mixing, and the chemical composition, $\mathrm{pH}$, conductivity, surface tension, and density of the extracts were determined. The effects on the composition and physical properties of the extracts, produced by the addition of small amounts of various materials to the pastes, were studied in parallel experiments. The added materials included gypsum, which was used in preparing the cements from the clinkers, calcium chloride, calcium acetate, fluosilicic acid, sucrose, T D A, tannic acid, and triethanolamine.

In the majority of the extracts, soda and potash were present in greater amounts than any of the other dissolved oxides. Relatively high concentrations of sulfate occurred in the extracts of the clinkers that contained the larger amounts of potash. The lime concentration and basicity of the extracts were largely determined by the amounts of alkali oxides present. The concentration of silica corresponded approximately to the reported solubility of silica in lime solutions. Low concentrations of oxides of the $\mathrm{R}_{2} \mathrm{O}_{3}$ group were found except in extracts from pastes containing the added organic materials.

\section{CONTENTS}

$\begin{array}{rr}\text { Page } \\ \text { I. Introduction } & 216\end{array}$

II. Materials

1. Clinkers _.

2. Addition agents

III. Experimental procedure 218

1. Preparation and filtration of pastes

2. Chemical analyses of filtrates...

3. $\mathrm{pH}$ and other constants of filtrates

IV. Results and discussion

1. General experimental results_.....

2. Composition of the filtrates

(a) Alkalies $\left(\mathrm{K}_{2} \mathrm{O}\right.$ and $\left.\mathrm{Na}_{2} \mathrm{O}\right)$

(b) Lime...

(c) Silica

(d) $\mathrm{R}_{2} \mathrm{O}_{3}$ group $\left(\mathrm{Al}_{2} \mathrm{O}_{3}, \mathrm{Fe}_{2} \mathrm{O}_{3} \text {, and } \mathrm{Cr}_{2} \mathrm{O}_{3}\right)_{\ldots}$

(e) $\mathrm{SO}_{3}$

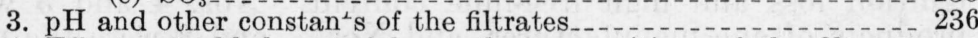

4. Effects of added materials on the compositions of the filtrates and on some of the reactions in hydrating clinkers _....... 240

(a) Gypsum

(b) Calcium chloride

(c) Calcium acetate

(d) Fluosilicic acid

(e) Sucrose

(f) $\mathrm{T} \mathrm{D} \mathrm{A}$

(g) Tannic acid

(h) Triethanolamine

V. Summary

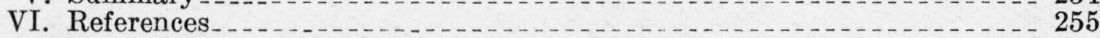




\section{INTRODUCTION}

A comprehensive series of investigations was undertaken at this Bureau to provide much needed information on the character of the liquid phase following the gaging of portland cements with water, both in the absence and in the presence of various materials that have been suggested for the control of the reactions of hydration. The important variables in the study included:

1. Compositions of the cement clinkers.

2. Presence of additive materials.

3 . Time of the reactions.

The first report on the series [1] ${ }^{1}$ presented observed relations between the potash and the sulfur trioxide contents of the clinkers and of the extracts, leading to the belief that potash is present in many clinkers partly as potassium sulfate. The present (second) report gives information on the effects of clinker composition, character of material added, and time of reaction on the composition, surface tension, density, hydrogen-ion concentration $(\mathrm{pH})$, and specific conductivity of the extracts. The third report [2] includes data on the effects of these same variables on the heats of hydration of the clinkers, the flows and strengths of specimens fabricated from the pastes after removal of the extracts, and the results of certain miscellaneous tests on the clinkers.

Because the amounts of extractable soda and potash in cements and their possible effects on the properties of cement products have been much discussed subjects for many years, the clinkers used in this study were chosen to give a large range in soda and potash contents. The materials added to the clinkers were gypsum, calcium chloride, calcium acetate, fluosilicic acid, sucrose, T D A, tannic acid, and triethanolamine. These substances are referred to as "added materials" or "addition agents" rather than as "admixtures."

\section{MATERIALS}

\section{CLINKERS}

The chemical analyses and computed compound compositions of the clinkers [3] are presented in table 1 . It is to be noted that the soda content varied from 0.02 to 0.87 percent and the potash content from 0.05 to 1.32 percent.

The clinkers represented standard (Nos. 1, 2, 3, 4, 6, 7, 11, and 12), moderate-heat-of-hardening (Nos. 5, 8, and 9), and sulfate-resisting (No. 10) types. No. 2, a white clinker (low $\mathrm{Fe}_{2} \mathrm{O}_{3}$ ), had a high ignition loss and high free-lime content. Nos. 4 and 5 were the products of one manufacturer, and Nos. 7 and 8 were the products of another manufacturer. All the remaining clinkers were obtained from different plants.

The clinkers, in 600-lb lots, were ground to the finenesses reported in table 1, as determined by Wagner's method [4]. The experiments on a given clinker were nearly completed before the next clinker was ground.

\footnotetext{
1 Figures in brackets indicate the literature references at the end of this paper.
} 
$\mathrm{T}_{\mathrm{ABLE}}$ 1.-Chemical analyses, potential compound compositions and finenesses of ground clinkers

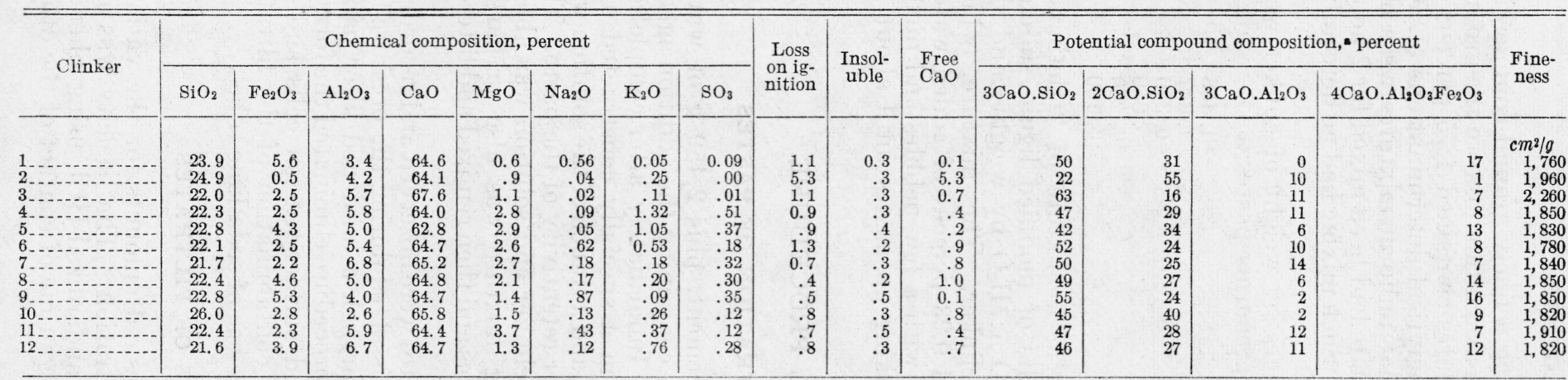

2 Potential values calculated with corrections only for free $\mathrm{CaO}$. 
aliquots of the basic solutions with glass receptacles prior to addition of $\mathrm{HCl}$ was about 20 minutes. The solutions were analyzed for the following constituents: $\mathrm{SiO}_{2}, \mathrm{R}_{2} \mathrm{O}_{3}, \mathrm{CaO}, \mathrm{MgO}, \mathrm{SO}_{3}, \mathrm{Na}_{2} \mathrm{O}$, and $\mathrm{K}_{2} \mathrm{O}$. The amounts of $\mathrm{Al}_{2} \mathrm{O}_{3}$ and $\mathrm{Fe}_{2} \mathrm{O}_{3}$ were generally determined when the $\mathrm{R}_{2} \mathrm{O}_{3}$ precipitate was comparatively large. $\mathrm{Cr}_{2} \mathrm{O}_{3}$ was estimated when its presence was indicated by a green color of the ignited $\mathrm{R}_{2} \mathrm{O}_{3}$. In addition to these, the amounts of $\mathrm{Cl}, \mathrm{CH}_{3} \mathrm{COO}$, and $\mathrm{F}$ were determined when calcium chloride, calcium acetate, and fluosilicic acid, respectively, had been added to the mixing water.

Standard procedures of analysis were followed, blank determinations being made and proper corrections applied. $\mathrm{SiO}_{2}, \mathrm{R}_{2} \mathrm{O}_{3}, \mathrm{CaO}, \mathrm{MgO}$, $\mathrm{Na}_{2} \mathrm{O}, \mathrm{K}_{2} \mathrm{O}, \mathrm{SO}_{3}$, and $\mathrm{Cl}$ were determined gravimetrically. $\mathrm{Cr}_{2} \mathrm{O}_{3}$ was estimated colorimetrically. $\mathrm{Fe}_{2} \mathrm{O}_{3}$ was determined volumetrically by titration with $\mathrm{K}_{2} \mathrm{Cr}_{2} \mathrm{O}_{7}$. $\quad \mathrm{Na}_{2} \mathrm{O}$ was precipitated and weighed as sodium uranyl zinc acetate. $\mathrm{K}_{2} \mathrm{O}$ was precipitated as potassium chlorplatinate, which was reduced to $\mathrm{Pt}$ and the $\mathrm{Pt}$ weighed. Acetate was determined titrametrically on distillates from mixtures of the original solutions plus $\mathrm{H}_{3} \mathrm{PO}_{4}[5]$. Fluorine was determined according to a method reported by Hoffman and Lundell [6].

\section{3. $\mathrm{pH}$ AND OTHER CONSTANTS OF FILTRATES}

Immediately after filtration, portions of the solutions were transferred to the receptacles used in the determination of $\mathrm{pH}$, conductivity, surface tension, and density. These were placed in an air bath, which maintained the temperature of the solutions at $25^{\circ} \pm 0.03^{\circ} \mathrm{C}$.

The determinations of $\mathrm{pH}$ were made by measuring the emf of the cell, $E_{c}$,

$$
\mathrm{Pt}(\mathrm{Pd} \text { black }) \mathrm{H}_{2}(\mathrm{~g}) \mid \mathrm{H}^{+} \text {(solution) } \mid \mathrm{KCl} \text { (saturated) } \mathrm{H}_{2} \underset{E_{2}}{\mathrm{Hg}_{2} \mathrm{Cl}_{2} \mid \mathrm{Hg}}
$$

at $25^{\circ} \mathrm{C}$ with a calibrated potentiometer, using a sensitive galvanometer and a standard cell. Details of the apparatus, including the cell setup, have been described [7]. The $\mathrm{pH}$ values of the solutions, after the values of $\mathrm{E}_{c}$ had been measured, were computed from the relation,

$$
\mathrm{pH}=\frac{E_{c}-\left(E_{1}+E_{2}+E_{0}\right)}{0.05914},
$$

in which $E_{0}$ is the standard potential of the saturated calomel electrode, and $E_{1}$ and $E_{2}$ represent the potentials that are developed at the liquid junctions designated in the cell setup.

The values of the liquid-junction potentials, $E_{1}$ and $E_{2}$, may be approximated by the use of the modified Henderson equation [8]. However, the computations of the value of $E_{1}$ are extensive and yield only a fair approximation for complex solutions such as those obtained in this study. Instead of using a reported value for $E_{0}$ and correcting for the liquid junction potentials by Henderson's equation, an alternate method was employed as described below.

The cell was standardized against solutions of potassium hydroxide, the $\mathrm{pH}$ values of which were computed by Hamer $[9]^{3}$ and are given in table 2 . The voltages, $E_{\mathrm{c}}$, of the cell for various concentrations of potassium hydroxide solution, ranging from 0.05 to $0.5 \mathrm{~N}$ were measured. The standard $\mathrm{pH}$ (designated as $\mathrm{pH}_{s}$ ) for each concen-

\footnotetext{
${ }^{3}$ In computing the $\mathrm{pH}$ values, Hamer used the best reported values [10] for the activity coefficients of potassium hydroxide, and the ionization constant of water, $K_{w}$, equal to $1.008 \times 10^{-14}$ at $25^{\circ} \mathrm{C}$ [11].
} 
tration of potassium hydroxide was then obtained from a $\mathrm{pH}_{3}$ curve of Hamer's data. Having the value of this standard $\mathrm{pH}_{s}$ and the emf of the cell, $E_{c}$, for a given potassium hydroxide solution, the voltage of the calomel electrode, including the two liquid-junction potentials $\left(E_{0}+E_{1}+E_{2}\right)$ represented by $\mathrm{E}_{c a l}$ was computed from the relation

$$
E_{c a l}=E_{c}-0.05914 \mathrm{pH}_{s} \text {. }
$$

TABLE 2.-Concentrations of solutions of potassium hydroxide and their $\mathrm{pH}$ values computed by Hamer [9]

\begin{tabular}{|c|c||c|c||c|c|}
\hline Concentration & $\mathrm{pH}$ & Concentration & $\mathrm{pH}$ & Concentration & $\mathrm{pH}$ \\
\cline { 2 - 5 } $\begin{array}{c}\text { Moles/1,000 } \\
\text { g of solvent }\end{array}$ & & $\begin{array}{c}\text { Moles } / 1,000 \\
\text { g of solvent }\end{array}$ & & $\begin{array}{c}\text { Moles/1,000 } \\
\text { g of solvent }\end{array}$ & \\
0.05 & 12.61 & 0.35 & 13.41 & 2.00 & 14.25 \\
.10 & 12.90 & .50 & 13.56 & 3.00 & 14.51 \\
.15 & 13.06 & 1.75 & 13.74 & 4.00 & 14.73 \\
.25 & 13.27 & 1.00 & 13.88 & & \\
\hline
\end{tabular}

The $E_{c a l}$ values for the range of potassium hydroxide concentrations from 0.05 to $0.5 \mathrm{~N}$ were found to vary systematically between 0.2346 and $0.2354 \mathrm{v}$. The extremes of this range represent a difference in $\mathrm{pH}$ of 0.01 units. Since the use of the average value for $E_{c a l}$ of $0.2350 \mathrm{v}$ would introduce a maximum error of only $0.01 \mathrm{pH}$ unit, all reported values of $\mathrm{pH}$ were computed from the equation

$$
\mathrm{pH}=\frac{E_{c}-0.2350}{0.05914} \text {. }
$$

During use, the cell setup was readily checked against a solution of $\mathrm{KOH}$ of known strength. Such calibration was found to be far more satisfactory than that obtained by the use of potassium acid phthalate buffer.

Conductivities were measured with calibrated equipment, and the results are accurate to about 0.1 percent.

The capillary-rise method was employed in the surface-tension measurements, the cell being calibrated with water, using the value of 71.98 dynes/cm [12]. The results on solutions of pure salts were reproducible to $\pm 0.03 \mathrm{dyne} / \mathrm{cm}$, but the filtrates from the clinker pastes frequently contained surface contaminants, presumably traces of oil picked up during filtration, and therefore the errors for such solutions were high.

The densities of the filtrates, determined according to standard procedure and corrected to vacuum, are accurate to about 1 part in 10,000 .

\section{RESULTS AND DISCUSSION}

\section{GENERAL EXPERIMENTAL RESULTS}

The data for the filtrates are reported in table 3 . All the results are for single determinations only. However, in a few scattered instances duplicate analyses of a given filtrate were made for one or more of the following: $\mathrm{CaO}, \mathrm{SO}_{3}, \mathrm{Na}_{2} \mathrm{O}, \mathrm{K}_{2} \mathrm{O}$, and basicity. The results of these tests agreed within 1 percent or less of the amounts reported, except those for $\mathrm{Na}_{2} \mathrm{O}$ when present in small amounts or when present with much $\mathrm{K}_{2} \mathrm{O}$. These differences were generally larger, ranging up to 10 percent (clinker 4 ). 
TABLE 3.-Chemical composition and physical properties of the aqueous phase of Portland cement clinker pastes with and without added materials

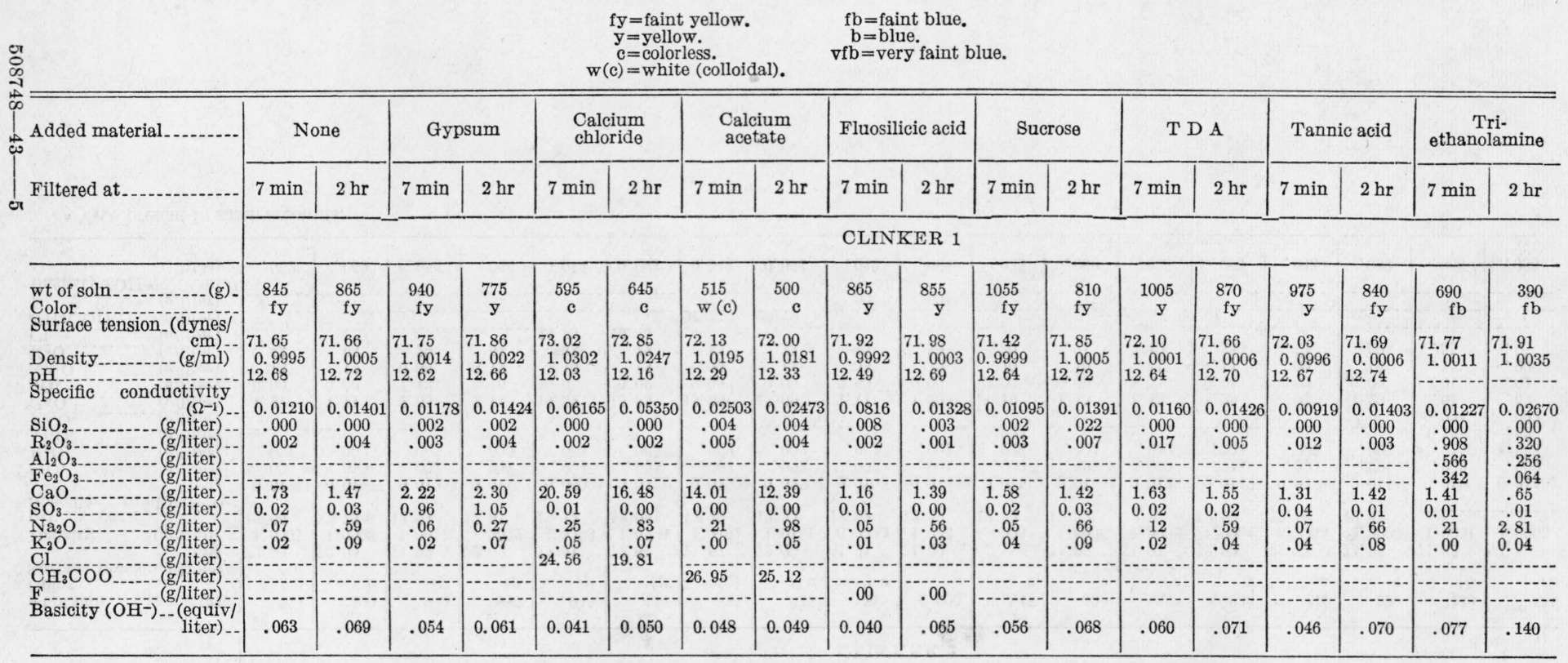


TABLE 3.-Chemical composition and physical properties of the aqueous phase of Portland cement clinker pastes with and without added materials-Continued

\begin{tabular}{|c|c|c|c|c|c|c|c|c|c|c|c|c|c|c|c|c|c|c|}
\hline \multirow{3}{*}{$\begin{array}{l}\text { Added material. } \\
\text { Filtered at. }\end{array}$} & \multicolumn{2}{|c|}{ None } & \multicolumn{2}{|c|}{ Gypsum } & \multicolumn{2}{|c|}{$\begin{array}{l}\text { Calcium } \\
\text { chloride }\end{array}$} & \multicolumn{2}{|c|}{$\begin{array}{l}\text { Calcium } \\
\text { acetate }\end{array}$} & \multicolumn{2}{|c|}{ Fluosilicic acid } & \multicolumn{2}{|c|}{ Sucrose } & \multicolumn{2}{|c|}{$\mathrm{T} \mathrm{DA}$} & \multicolumn{2}{|c|}{ Tannic acid } & \multicolumn{2}{|c|}{$\begin{array}{l}\text { Tri- } \\
\text { ethanolamine }\end{array}$} \\
\hline & $7 \mathrm{~min}$ & $2 \mathrm{hr}$ & $7 \mathrm{~min}$ & $2 \mathrm{hr}$ & $7 \mathrm{~min}$ & $2 \mathrm{hr}$ & $7 \mathrm{~min}$ & $2 \mathrm{hr}$ & $7 \mathrm{~min}$ & $2 \mathrm{hr}$ & $7 \mathrm{~min}$ & $2 \mathrm{hr}$ & $7 \mathrm{~min}$ & $2 \mathrm{hr}$ & $7 \mathrm{~min}$ & $2 \mathrm{hr}$ & $7 \mathrm{~min}$ & $2 \mathrm{hr}$ \\
\hline & \multicolumn{18}{|c|}{ CLINKER 2} \\
\hline $\begin{array}{l}\text { wt of soln } \\
\text { Color } \\
\text { Surface tension (dynes/ }\end{array}$ & $\begin{array}{r}660 \\
\mathrm{c}\end{array}$ & $\begin{array}{r}590 \\
\mathrm{c}\end{array}$ & $\begin{array}{r}980 \\
\text { fy }\end{array}$ & $\begin{array}{r}835 \\
\text { fy }\end{array}$ & $\begin{array}{r}945 \\
\text { fy }\end{array}$ & $\begin{array}{r}435 \\
\text { fy }\end{array}$ & $\begin{array}{r}835 \\
\mathrm{w}(\mathrm{c})\end{array}$ & $\begin{array}{r}635 \\
\mathrm{c}\end{array}$ & $\begin{array}{r}615 \\
\mathrm{c}\end{array}$ & $\begin{array}{r}600 \\
\mathrm{c}\end{array}$ & $\begin{array}{r}845 \\
\mathrm{C}\end{array}$ & $\begin{array}{r}500 \\
\mathrm{c}\end{array}$ & $\begin{array}{r}855 \\
\text { fy }\end{array}$ & $\begin{array}{r}630 \\
\mathrm{y}\end{array}$ & $\begin{array}{r}235 \\
\mathrm{c}\end{array}$ & $\begin{array}{r}520 \\
\mathrm{c}\end{array}$ & $\begin{array}{c}430 \\
\mathrm{fb}\end{array}$ & \\
\hline $\begin{array}{l}\text { Density } \\
\text { pH. } \\
\text { Specific conductivity }\end{array}$ & $\begin{array}{l}72.00 \\
1.0001 \\
12.66\end{array}$ & $\begin{array}{l}72.04 \\
1.0005 \\
12.73\end{array}$ & \begin{tabular}{|l|} 
71. 99 \\
1.0017 \\
12.64
\end{tabular} & $\mid \begin{array}{l}72.02 \\
1.0022 \\
12.67\end{array}$ & \begin{tabular}{|l|}
72.91 \\
1.0293 \\
12.12
\end{tabular} & \begin{tabular}{|l|}
72.67 \\
1.0236 \\
12.09
\end{tabular} & $\begin{array}{l}\text { 71. } 67 \\
1.0191 \\
12.29\end{array}$ & \begin{tabular}{|l|}
72.23 \\
1.0181 \\
12.35
\end{tabular} & $\begin{array}{l}01.91 \\
0.9992 \\
12.59\end{array}$ & $\begin{array}{l}71.90 \\
1.003 \\
12.69\end{array}$ & $\begin{array}{l}1.0000 \\
12.62\end{array}$ & \begin{tabular}{|l|}
71.96 \\
1.0005 \\
12.72
\end{tabular} & \begin{tabular}{|l|}
71.91 \\
1.0002 \\
12.67
\end{tabular} & \begin{tabular}{|l|}
72.00 \\
1.0004 \\
12.69
\end{tabular} & \begin{tabular}{|l}
71.83 \\
0.9993 \\
12.53
\end{tabular} & $\left|\begin{array}{l}71.96 \\
1.0005 \\
12.67\end{array}\right|$ & \begin{tabular}{l}
71.85 \\
1.0001 \\
\hdashline-1
\end{tabular} & $\begin{array}{l}71.80 \\
1.0009\end{array}$ \\
\hline $\begin{array}{l}\text { ( } \begin{array}{r}(\Omega-1) \\
\text { (g/liter) }\end{array}- \\
\mathrm{SiO}_{2} \\
\mathrm{R}_{2} \mathrm{O}_{3-1} \\
\mathrm{Al}_{2} \mathrm{O}_{3}\end{array}$ & $\begin{array}{c}0.01261 \\
.000 \\
.001 \\
-. .-\end{array}$ & $\begin{array}{c}0.01477 \\
.000 \\
.002 \\
-. .--\end{array}$ & $\begin{array}{l}0.01258 \\
.002 \\
.005 \\
-.0\end{array}$ & $\begin{array}{l}0.01462 \\
.001 \\
.005\end{array}$ & $\begin{array}{c}0.06084 \\
.004 \\
.003 \\
\cdots\end{array}$ & \begin{tabular}{l}
0.05213 \\
.004 \\
.003 \\
\hdashline.-
\end{tabular} & $\begin{array}{c}0.02504 \\
.002 \\
.002\end{array}$ & $=\begin{array}{l}0.02502 \\
.003 \\
.002\end{array}$ & \begin{tabular}{l}
0.0100 \\
.003 \\
.002 \\
\hdashline..-
\end{tabular} & $\begin{array}{l}0.01360 \\
.001 \\
.001\end{array}$ & $\begin{array}{l}0.01112 \\
.000 \\
.024 \\
.024\end{array}$ & $\begin{array}{l}0.01454 \\
.001 \\
.004\end{array}$ & $\begin{array}{l}0.01295 \\
.000 \\
.002\end{array}$ & $\begin{array}{l}0.01425 \\
.013 \\
.003\end{array}$ & $\begin{array}{l}0.00906 \\
.000 \\
.005\end{array}$ & $\begin{array}{c}0.01446 \\
.001 \\
.003 \\
-\end{array}$ & $\begin{array}{l}0.01094 \\
.000 \\
\text { ×1. } 215 \\
1.16 \\
0.024\end{array}$ & $\begin{array}{l}0.01483 \\
.000 \\
\text { 8. } 668 \\
.66 \\
.007\end{array}$ \\
\hline $\begin{array}{l}\mathrm{Fe}_{2} \mathrm{O}_{3} \\
\mathrm{CaO} \\
\mathrm{SO}_{3} \\
\mathrm{Na}_{2} \mathrm{O} \\
\mathrm{K}_{2} \mathrm{O} \\
\mathrm{Cl} \text { (g/liter) }\end{array}$ & $\begin{array}{r}1.27 \\
0.00 \\
.13 \\
.43\end{array}$ & $\begin{array}{r}1.11 \\
0.00 \\
.19 \\
.95\end{array}$ & $\begin{array}{l}2.00 \\
1.13 \\
0.09 \\
.29\end{array}$ & $\begin{array}{l}2.14 \\
1.16 \\
0.12 \\
.54\end{array}$ & $\begin{array}{r}19.92 \\
0.03 \\
.10 \\
.33 \\
23.78\end{array}$ & $\begin{array}{r}15.85 \\
0.02 \\
.15 \\
.72 \\
19.35\end{array}$ & $\begin{array}{r}13.66 \\
0.00 \\
.09 \\
.45\end{array}$ & $\begin{array}{r}12.60 \\
0.00 \\
.14 \\
.73\end{array}$ & $\begin{array}{r}1.11 \\
0.00 \\
.06 \\
.40\end{array}$ & $\begin{array}{l}1.20 \\
0.00 \\
.12 \\
.89\end{array}$ & $\begin{array}{l}1.19 \\
0.00 \\
.10 \\
.30\end{array}$ & $\begin{array}{r}1.15 \\
0.00 \\
.19 \\
.71\end{array}$ & $\begin{array}{r}1.42 \\
0.01 \\
.11 \\
.49\end{array}$ & $\begin{array}{l}1.26 \\
0.01 \\
.17 \\
.88\end{array}$ & $\begin{array}{l}.93 \\
.00 \\
.09 \\
.13\end{array}$ & $\begin{array}{l}1.20 \\
0.00 \\
.16 \\
.69\end{array}$ & $\begin{array}{l}0.024 \\
.61 \\
.01 \\
.11 \\
.55\end{array}$ & $\begin{array}{l}.007 \\
.52 \\
.01 \\
.20 \\
.95\end{array}$ \\
\hline $\mathrm{CH}_{3} \mathrm{COO}-$ (g/liter) & & 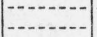 & (n) & -.. & & & 27.11 & 25.12 & & & & & & & & & & \\
\hline $\begin{array}{r}\text { Basicity }\left(\mathrm{OH}^{-}\right) \text {(liter) } \\
\text { liter) } \\
\text { live }\end{array}$ & .062 & .069 & .056 & .066 & 0.048 & 0.043 & 0.048 & 0.051 & $\begin{array}{l}.001 \\
.049\end{array}$ & $\begin{array}{l}.001 \\
.065\end{array}$ & .055 & .069 & .065 & .069 & .043 & .069 & .066 & .083 \\
\hline
\end{tabular}

- $\mathrm{V}_{2} \mathrm{O}_{3}$ present in small amounts. 
OLINKER 3

\begin{tabular}{|c|c|c|c|c|c|c|c|c|c|c|c|c|c|c|c|c|c|c|}
\hline $\begin{array}{l}\text { wt of soln } \\
\text { Color } \\
\text { Surface tension }\end{array}$ & $\begin{array}{r}710 \\
c\end{array}$ & $\begin{array}{r}725 \\
\mathrm{c}\end{array}$ & $\begin{array}{l}820 \\
\text { fy }\end{array}$ & $\begin{array}{r}625 \\
\text { fy }\end{array}$ & $\begin{array}{r}640 \\
\text { fy }\end{array}$ & $\begin{array}{r}440 \\
\mathrm{c}\end{array}$ & $\begin{array}{r}505 \\
(w) \mathrm{c}\end{array}$ & $\begin{array}{r}285 \\
\mathrm{c}\end{array}$ & $\begin{array}{r}505 \\
\mathrm{c}\end{array}$ & $\begin{array}{r}675 \\
\mathrm{c}\end{array}$ & $\begin{array}{r}615 \\
\mathrm{c}\end{array}$ & $\begin{array}{r}685 \\
\mathrm{c}\end{array}$ & $\begin{array}{r}665 \\
\mathrm{c}\end{array}$ & $\begin{array}{r}685 \\
\mathrm{c}\end{array}$ & $\begin{array}{r}525 \\
\mathrm{c}\end{array}$ & $\begin{array}{r}600 \\
\mathrm{c}\end{array}$ & $\begin{array}{r}265 \\
\mathrm{~b}\end{array}$ & $\begin{array}{r}465 \\
b\end{array}$ \\
\hline $\begin{array}{l}\text { (dynes/cm) } \\
\text { Density } \\
\text { pH }\end{array}$ & $\begin{array}{l}72.040 \\
1.002 \\
12.67\end{array}$ & $\mid \begin{array}{l}72.00 \\
1.0002 \\
12.67\end{array}$ & \begin{tabular}{|l|}
71.93 \\
1.0019 \\
12.63
\end{tabular} & $\left|\begin{array}{l}72.00 \\
1.0016 \\
12.61\end{array}\right|$ & $\begin{array}{l}72.75 \\
1.0265 \\
12.17\end{array}$ & \begin{tabular}{|l|}
72.56 \\
1.0196 \\
12.15
\end{tabular} & $\begin{array}{l}1.0135 \\
12.41\end{array}$ & $\begin{array}{l}1.0108 \\
12.27\end{array}$ & $\begin{array}{l}71.83 \\
0.9999 \\
12.62\end{array}$ & \begin{tabular}{|l}
71.92 \\
1.0002 \\
12.60
\end{tabular} & $\begin{array}{l}72.00 \\
1.0003 \\
12.68\end{array}$ & \begin{tabular}{|l|}
71.98 \\
1.0003 \\
12.68
\end{tabular} & 1.0001 & 1.0007 & $\begin{array}{l}1.0001 \\
12.67\end{array}$ & $\begin{array}{l}1.0000 \\
12.66\end{array}$ & 1.0008 & 1.0011 \\
\hline $\begin{array}{l}\text { Specific conductivity } \\
\left(\Omega^{-1}\right) \\
\mathrm{SiO}_{2} \\
\mathrm{R}_{2} \mathrm{O}_{3} \\
\mathrm{Al}_{2} \mathrm{O}_{3} \\
\mathrm{Fe}_{2} \mathrm{O}_{3} \\
\text { (g/liter) }\end{array}$ & $\begin{array}{c}0.01252 \\
.001 \\
.004 \\
-0 .\end{array}$ & $\begin{array}{l}0.01251 \\
.000 \\
.005\end{array}$ & $\begin{array}{c}0.01323 \\
.001 \\
.004\end{array}$ & \begin{tabular}{l}
0.01272 \\
.000 \\
.006 \\
\hdashline-0
\end{tabular} & $\begin{array}{l}0.05650 \\
.000 \\
.005 \\
-. . .\end{array}$ & $\begin{array}{l}0.04495 \\
.004 \\
-\end{array}$ & $\begin{array}{l}0.02209 \\
.002 \\
.001 \\
-.0\end{array}$ & $\begin{array}{l}0.01873 \\
.002 \\
.003\end{array}$ & $\begin{array}{l}0.01098 \\
.001 \\
.003\end{array}$ & $\begin{array}{l}0.01166 \\
.000 \\
.001\end{array}$ & 0.01304 & 0.01298 & 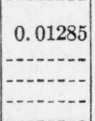 & \begin{tabular}{r}
0.01302 \\
\hdashline-2 \\
\end{tabular} & 0.01269 & 0.01262 & $\begin{array}{l}0.01004 \\
.002 \\
.273 \\
.154 \\
.119\end{array}$ & $\begin{array}{l}0.01501 \\
.001 \\
.137 \\
.113 \\
.024\end{array}$ \\
\hline 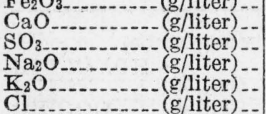 & $\begin{array}{l}1.58 \\
0.00 \\
.06 \\
.28\end{array}$ & $\begin{array}{r}1.52 \\
0.00 \\
.07 \\
.31\end{array}$ & $\begin{array}{l}2.34 \\
1.02 \\
0.05 \\
.17\end{array}$ & $\begin{array}{r}2.17 \\
1.03 \\
0.05 \\
.26\end{array}$ & $\begin{array}{r}18.40 \\
0.00 \\
.05 \\
.19 \\
21.17\end{array}$ & $\begin{array}{r}13.68 \\
0.00 \\
.11 \\
.39 \\
15.93\end{array}$ & $\begin{array}{r}10.01 \\
0.00 \\
.08 \\
.32\end{array}$ & $\begin{array}{r}8.07 \\
0.00 \\
.14 \\
.49\end{array}$ & $\begin{array}{l}1.44 \\
0.01 \\
.02 \\
.16\end{array}$ & $\begin{array}{r}1.43 \\
0.00 \\
.05 \\
.30\end{array}$ & $\begin{array}{r}1.58 \\
0.00 \\
.10 \\
.36\end{array}$ & $\begin{array}{r}1.48 \\
0.00 \\
.14 \\
.45\end{array}$ & $\begin{array}{r}1.64 \\
0.00 \\
.07 \\
.33\end{array}$ & $\begin{array}{r}1.57 \\
0.00 \\
.11 \\
.40\end{array}$ & $\begin{array}{r}1.60 \\
0.00 \\
.08 \\
.31\end{array}$ & $\begin{array}{l}1.50 \\
0.00 \\
.09 \\
.37\end{array}$ & $\begin{array}{l}.119 \\
1.16 \\
0.00 \\
.10 \\
.39\end{array}$ & $\begin{array}{l}.024 \\
1.51 \\
0.00 \\
.18 \\
.68\end{array}$ \\
\hline $\mathrm{CH}_{3} \mathrm{COO}$ (g/liter) & & & & & & & 18.13 & 15.38 & 00 & 00 & & & & & & & & \\
\hline $\begin{array}{r}\text { Basicity }\left(\mathrm{OH}^{-}\right) \\
\text {(equiv/liter) }\end{array}$ & .064 & .063 & .063 & .059 & 0.056 & 0.045 & 0.055 & 0.038 & $\begin{array}{l}.00 \\
.056\end{array}$ & .058 & .066 & .065 & .066 & .065 & .065 & .063 & .068 & .086 \\
\hline \multicolumn{19}{|c|}{ CLINKER 4} \\
\hline $\begin{array}{l}\text { wt of soln } \\
\text { Color } \\
\text { Surface tension }\end{array}$ & $\begin{array}{r}660 \\
\mathrm{y}\end{array}$ & $\begin{array}{r}600 \\
\mathrm{y}\end{array}$ & $\begin{array}{r}670 \\
\mathrm{y}\end{array}$ & $\begin{array}{r}665 \\
\mathrm{y}\end{array}$ & $\begin{array}{r}580 \\
\mathrm{c}\end{array}$ & $\begin{array}{r}620 \\
\mathrm{C}\end{array}$ & $\begin{array}{r}515 \\
\mathrm{C}\end{array}$ & $\begin{array}{r}475 \\
\mathrm{c}\end{array}$ & $\begin{array}{r}535 \\
\mathrm{y}\end{array}$ & $\begin{array}{r}535 \\
\mathrm{y}\end{array}$ & $\begin{array}{r}405 \\
c\end{array}$ & $\begin{array}{r}450 \\
\mathrm{c}\end{array}$ & $\begin{array}{r}735 \\
\mathrm{y}\end{array}$ & $\begin{array}{r}695 \\
\mathrm{y}\end{array}$ & $\begin{array}{r}285 \\
\mathrm{y}\end{array}$ & $\begin{array}{r}365 \\
\text { fy }\end{array}$ & & $\begin{array}{r}265 \\
\mathrm{~b}\end{array}$ \\
\hline $\begin{array}{l}\text { Density (dynes/cm) } \\
\text { pH.- } \\
\text { Specific conductivity }\end{array}$ & \begin{tabular}{|l|}
72.26 \\
1.0216 \\
13.19
\end{tabular} & \begin{tabular}{|l|}
72.28 \\
1.0206 \\
13.41
\end{tabular} & \begin{tabular}{|l}
72.27 \\
1.0239 \\
12.99
\end{tabular} & $\mid \begin{array}{l}72.25 \\
1.0254 \\
13.07\end{array}$ & $\begin{array}{l}72.88 \\
1.0295 \\
12.32\end{array}$ & \begin{tabular}{|l|}
72.85 \\
1.0272 \\
12.44
\end{tabular} & \begin{tabular}{|l|}
72.51 \\
1.0224 \\
12.91
\end{tabular} & \begin{tabular}{|l|}
72.33 \\
1.0235 \\
12.93
\end{tabular} & \begin{tabular}{|l}
72.18 \\
2.0187 \\
13.13
\end{tabular} & \begin{tabular}{|l|}
72.66 \\
1.0192 \\
13.46
\end{tabular} & \begin{tabular}{|l|}
72.25 \\
1.0218
\end{tabular} & \begin{tabular}{|l}
72.75 \\
1.0232 \\
13.58
\end{tabular} & \begin{tabular}{|l}
72.33 \\
1.0235 \\
13.00
\end{tabular} & $\begin{array}{l}72.34 \\
1.0216 \\
13.30\end{array}$ & \begin{tabular}{|l}
72.21 \\
1.0198 \\
13.26
\end{tabular} & \begin{tabular}{|l|}
72.30 \\
1.0213 \\
13.49
\end{tabular} & & $\begin{array}{l}\text { 72. } 58 \\
1.0256\end{array}$ \\
\hline $\begin{array}{l}\text { (g/liter) } \\
\mathrm{SiO}_{2} \\
\mathrm{R}_{2} \mathrm{O}_{3} \\
\mathrm{Al}_{2} \mathrm{O}_{3}-\mathrm{O}_{3} \\
\mathrm{Fe}_{2} \mathrm{O}_{3-} \text { (g/liter) } \\
\text { (g/liter) } \\
-\end{array}$ & $\begin{array}{l}0.06565 \\
.025 \\
.033\end{array}$ & $\begin{array}{l}0.08800 \\
.012 \\
.018\end{array}$ & $\begin{array}{l}0.05445 \\
.008 \\
.060\end{array}$ & \begin{tabular}{l}
0.06040 \\
.008 \\
.061 \\
\hdashline-0 \\
-0
\end{tabular} & $\begin{array}{c}0.07520 \\
.008 \\
.006\end{array}$ & $\begin{array}{c}0.07380 \\
.007 \\
.001 \\
-0 \\
-0\end{array}$ & $\begin{array}{l}0.05310 \\
.00 \\
.002\end{array}$ & $\begin{array}{c}0.05545 \\
.00 \\
.007\end{array}$ & $\begin{array}{c}0.05810 \\
.007 \\
.045\end{array}$ & $\begin{array}{c}0.09360 \\
.001 \\
.007\end{array}$ & $\begin{array}{c}0.1102 \\
.016 \\
.231 \\
.231\end{array}$ & $\begin{array}{l}0.1172 \\
.065 \\
.019 \\
.019\end{array}$ & $\begin{array}{c}0.05484 \\
.005 \\
.043 \\
\end{array}$ & \begin{tabular}{c}
0.07420 \\
.011 \\
.041 \\
\hdashline-0 \\
-0
\end{tabular} & $\begin{array}{c}0.06790 \\
.047 \\
.057\end{array}$ & $\begin{array}{c}0.1092 \\
.031 \\
.011\end{array}$ & & $\begin{array}{l}0.1232 \\
.059 \\
.286 \\
.051 \\
.235\end{array}$ \\
\hline $\mathrm{Cr}_{2} \mathrm{O}_{3}$ & .030 & .015 & .050 & .050 & & & & & .025 & .005 & & & .035 & .035 & .025 & & & \\
\hline - (g/liter) & .23 & .21 & 1.00 & 1.05 & 7.13 & 4.61 & 1.69 & 1.26 & .12 & .05 & .05 & .10 & .66 & .35 & .10 & .11 & & .07 \\
\hline $\mathrm{SO}_{3}$ & 7.90 & 3.35 & 11. 72 & 11. 74 & 0.04 & 0.01 & 0.04 & 0.05 & 6.89 & .56 & .10 & .10 & 11. 40 & 6.57 & 6.14 & .16 & & .06 \\
\hline $\begin{array}{l}\mathrm{Na}_{2} \mathrm{O} \\
\mathrm{K}_{2} \mathrm{O}\end{array}$ & $\begin{array}{r}0.48 \\
18.65\end{array}$ & $\begin{array}{r}0.79 \\
19.71\end{array}$ & $\begin{array}{r}0.35 \\
18.23\end{array}$ & $\begin{array}{r}0.52 \\
19.34\end{array}$ & $\begin{array}{r}.42 \\
21.08\end{array}$ & 22.74 & $\begin{array}{r}.33 \\
21.74\end{array}$ & $\begin{array}{r}.48 \\
23.46\end{array}$ & $\begin{array}{r}0.12 \\
17.02\end{array}$ & .21 & 22.75 & 23.92 & $\begin{array}{r}0.20 \\
18.18\end{array}$ & $\begin{array}{r}0.26 \\
19.63\end{array}$ & $\begin{array}{r}0.28 \\
18.30\end{array}$ & .28 & (n). & $\begin{array}{r}2.47 \\
25.77\end{array}$ \\
\hline $\mathrm{Cl}$ & & & & & 23.61 & 21.65 & & 23.40 & & & & & & & & & & 25.77 \\
\hline $\mathrm{CH}_{3} \mathrm{COO}$ & -- & & & & & & 25.46 & 26.38 & & & & & & & & & & \\
\hline $\begin{array}{c}\text { Basicity (equiv/liter) } \\
\left(\mathrm{OH}^{-}\right)^{(\mathrm{g} / \text { liter) }}\end{array}$ & 0.213 & 0.358 & 0.134 & 0.162 & 0.048 & 0.052 & 0.103 & 0.102 & $\begin{array}{l}0.11 \\
.184\end{array}$ & $\begin{array}{l}0.18 \\
.397\end{array}$ & 0.492 & 0.524 & 0.135 & 0.276 & 0.240 & 0.485 & & 0.570 \\
\hline
\end{tabular}


TABLE 3.-Chemical composition and physical properties of the aqueous phase of Portland cement clinker pastes with and without added materials-Continued

\begin{tabular}{|c|c|c|c|c|c|c|c|c|c|c|c|c|c|c|c|c|c|c|}
\hline \multirow{3}{*}{$\begin{array}{l}\text { Added material } \\
\text { Filtered at. }\end{array}$} & \multicolumn{2}{|c|}{ None } & \multicolumn{2}{|c|}{ Gypsum } & \multicolumn{2}{|c|}{$\begin{array}{l}\text { Calcium } \\
\text { chloride }\end{array}$} & \multicolumn{2}{|c|}{$\begin{array}{l}\text { Calcium } \\
\text { acetate }\end{array}$} & \multicolumn{2}{|c|}{ Fluosilicic acid } & \multicolumn{2}{|c|}{ Sucrose } & \multicolumn{2}{|c|}{ T D A } & \multicolumn{2}{|c|}{ Tannic acid } & \multicolumn{2}{|c|}{$\begin{array}{l}\text { Tri- } \\
\text { ethanolamine }\end{array}$} \\
\hline & $7 \mathrm{~min}$ & $2 \mathrm{hr}$ & $7 \mathrm{~min}$ & $2 \mathrm{hr}$ & $7 \mathrm{~min}$ & $2 \mathrm{hr}$ & $7 \mathrm{~min}$ & $2 \mathrm{hr}$ & $7 \mathrm{~min}$ & $2 \mathrm{hr}$ & $7 \mathrm{~min}$ & $2 \mathrm{hr}$ & $7 \mathrm{~min}$ & $2 \mathrm{hr}$ & $7 \mathrm{~min}$ & $2 \mathrm{hr}$ & $7 \mathrm{~min}$ & $2 \mathrm{hr}$ \\
\hline & \multicolumn{18}{|c|}{ CLINKER 5} \\
\hline $\begin{array}{l}\text { wt of soln } \\
\text { Color } \\
\text { Surface tension }\end{array}$ & $\begin{array}{r}495 \\
\mathbf{y}\end{array}$ & $\begin{array}{r}605 \\
\mathrm{c}\end{array}$ & $\underset{\mathrm{y}}{620}$ & $\begin{array}{r}660 \\
\mathrm{y}\end{array}$ & $\begin{array}{r}625 \\
\mathrm{c}\end{array}$ & $\begin{array}{r}575 \\
\mathrm{c}\end{array}$ & $\begin{array}{r}425 \\
\mathrm{c}\end{array}$ & $\begin{array}{r}630 \\
\mathrm{c}\end{array}$ & $\begin{array}{r}405 \\
\mathrm{y}\end{array}$ & $\begin{array}{r}465 \\
\mathrm{c}\end{array}$ & $\begin{array}{r}245 \\
\mathrm{c}\end{array}$ & $\begin{array}{r}520 \\
\mathrm{c}\end{array}$ & $\begin{array}{r}635 \\
\mathrm{~J}\end{array}$ & $\begin{array}{r}735 \\
\mathrm{y}\end{array}$ & $\begin{array}{r}285 \\
\mathrm{y}\end{array}$ & $\begin{array}{r}465 \\
\mathrm{c}\end{array}$ & & $\begin{array}{r}325 \\
\mathrm{~b}\end{array}$ \\
\hline $\begin{array}{l}\text { (dynes/cm) } \\
\text { Density } \\
\text { pH } \\
\text { Specific conductivity }\end{array}$ & $\begin{array}{l}72.20 \\
1.0126 \\
13.19\end{array}$ & $\begin{array}{l}\text { 72. } 02 \\
1.0133 \\
13.39\end{array}$ & $\begin{array}{l}72.08 \\
1.0159 \\
12.91\end{array}$ & \begin{tabular}{|l}
72.24 \\
1.0171 \\
13.04
\end{tabular} & $\begin{array}{l}72.95 \\
1.0297 \\
12.24\end{array}$ & \begin{tabular}{|l|}
72.75 \\
1.0277 \\
12.29
\end{tabular} & $\begin{array}{l}72.28 \\
1.0193 \\
12.54\end{array}$ & $\begin{array}{l}1.0205 \\
12.68\end{array}$ & $\begin{array}{l}71.92 \\
1.0112 \\
12.97\end{array}$ & \begin{tabular}{|l}
72.11 \\
1.0134 \\
13.38
\end{tabular} & $\begin{array}{l}72.09 \\
1.0128 \\
13.31\end{array}$ & $\begin{array}{l}72.50 \\
1.0154 \\
13.43\end{array}$ & $\begin{array}{l}72.12 \\
1.0154 \\
12.91\end{array}$ & $\begin{array}{l}72.29 \\
1.0150 \\
13.13\end{array}$ & $\begin{array}{l}71.96 \\
1.0126 \\
13.08\end{array}$ & $\begin{array}{l}72.39 \\
1.0145 \\
13.39\end{array}$ & & \begin{tabular}{l}
72.05 \\
1.0167 \\
\hdashline
\end{tabular} \\
\hline $\begin{array}{l}\text { specinc conductivity } \\
\left.\text { ( } \Omega^{-1}\right) \\
\mathrm{SiO}_{2} \\
\mathrm{R}_{2} \mathrm{O}_{3} \\
\mathrm{Al}_{3} \mathrm{O}_{3} \\
\mathrm{Fe}_{2} \mathrm{O}_{3} \text { (g/liter) } \\
\text { (g/liter) } \\
-\end{array}$ & $\begin{array}{c}0.05520 \\
.029 \\
.023 \\
- \\
-\end{array}$ & \begin{tabular}{l}
0.07555 \\
.015 \\
.005 \\
\hdashline$-0 .-$ \\
..--
\end{tabular} & $\begin{array}{l}0.04160 \\
.005 \\
.031\end{array}$ & \begin{tabular}{c}
0.04870 \\
.004 \\
.029 \\
\hdashline-0 \\
-
\end{tabular} & \begin{tabular}{c}
0.07070 \\
.003 \\
.007 \\
\hdashline
\end{tabular} & $\begin{array}{l}0.06875 \\
.002 \\
.006 \\
-\end{array}$ & \begin{tabular}{c}
0.03928 \\
.007 \\
.007 \\
\hdashline \\
\end{tabular} & \begin{tabular}{c}
0.04152 \\
.000 \\
.003 \\
\hdashline \\
-
\end{tabular} & \begin{tabular}{c}
0.04085 \\
.006 \\
.030 \\
\hdashline
\end{tabular} & $\begin{array}{l}0.07390 \\
.000 \\
.026 \\
.026 \\
\end{array}$ & $\begin{array}{l}0.07045 \\
.028 \\
.283 \\
.283\end{array}$ & $\begin{array}{l}0.08415 \\
.020 \\
.013 \\
.013 \\
\end{array}$ & $\begin{array}{c}0.04120 \\
.007 \\
.042 \\
- \\
\end{array}$ & $\begin{array}{l}0.05275 \\
.022 \\
.027 \\
-.0\end{array}$ & $\begin{array}{c}0.04700 \\
.028 \\
.054\end{array}$ & $\begin{array}{l}0.07950 \\
.030 \\
.012\end{array}$ & & $\begin{array}{l}0.08395 \\
.048 \\
.500 \\
.045 \\
.455\end{array}$ \\
\hline $\begin{array}{l}\mathrm{Cr}_{2} \mathrm{O}_{3} \\
\mathrm{CaO}^{-(g / l i t e r} \text { (g/liter) } \\
\mathrm{SO}_{3} \\
\mathrm{Na}_{2} \mathrm{O} \\
\mathrm{K}_{2} \mathrm{O} \\
\mathrm{Cl}\end{array}$ & $\begin{array}{r}.010 \\
.12 \\
2.81 \\
0.11 \\
13.21\end{array}$ & $\begin{array}{r}.15 \\
.13 \\
.17 \\
15.08\end{array}$ & $\begin{array}{r}.030 \\
.96 \\
7.76 \\
0.09 \\
12.90\end{array}$ & $\begin{array}{l}.025 \\
1.09 \\
7.45 \\
0.13 \\
13.92\end{array}$ & $\begin{array}{r}11.48 \\
0.04 \\
.12 \\
14.15 \\
23.85\end{array}$ & $\begin{array}{r}9.49 \\
0.02 \\
.16 \\
15.89 \\
22.31\end{array}$ & $\begin{array}{r}4.69 \\
0.02 \\
.18 \\
14.71\end{array}$ & $\begin{array}{r}4.46 \\
0.01 \\
.23 \\
16.20\end{array}$ & $\begin{array}{l}.015 \\
.17 \\
3.99 \\
0.06 \\
11.49\end{array}$ & $\begin{array}{r}.06 \\
.11 \\
.20 \\
15.49\end{array}$ & $\begin{array}{r}.04 \\
.15 \\
.22 \\
14.38\end{array}$ & $\begin{array}{r}.13 \\
.08 \\
.34 \\
16.91\end{array}$ & $\begin{array}{r}.65 \\
7.53 \\
0.10 \\
13.09\end{array}$ & $\begin{array}{r}.42 \\
5.15 \\
0.14 \\
14.13\end{array}$ & $\begin{array}{r}.030 \\
.13 \\
4.08 \\
0.12 \\
12.84\end{array}$ & $\begin{array}{r}.10 \\
.09 \\
.42 \\
16.14\end{array}$ & 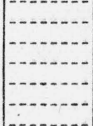 & $\begin{array}{r}.06 \\
.09 \\
.47 \\
17.72\end{array}$ \\
\hline $\mathrm{CH}_{3} \mathrm{COO}_{-}$(g/liter) & & $\cdots$ & -- & $\cdots$ & & & 24.49 & $25.91^{-}$ & & & & & & & & & & (n) \\
\hline $\begin{array}{c}\text { Basicity (equiv/liter) } \\
\left(\mathrm{O}-\mathrm{H}^{-}\right)\end{array}$ & 0.211 & 0.328 & 0.110 & 0.148 & 0.048 & 0.046 & 0.061 & 0.066 & $\begin{array}{l}0.09 \\
.132\end{array}$ & $\begin{array}{l}0.16 \\
.315\end{array}$ & 0.307 & 0.366 & 0.109 & 0.181 & 0.163 & 0.347 & & 0.381 \\
\hline
\end{tabular}


CLINKER 6

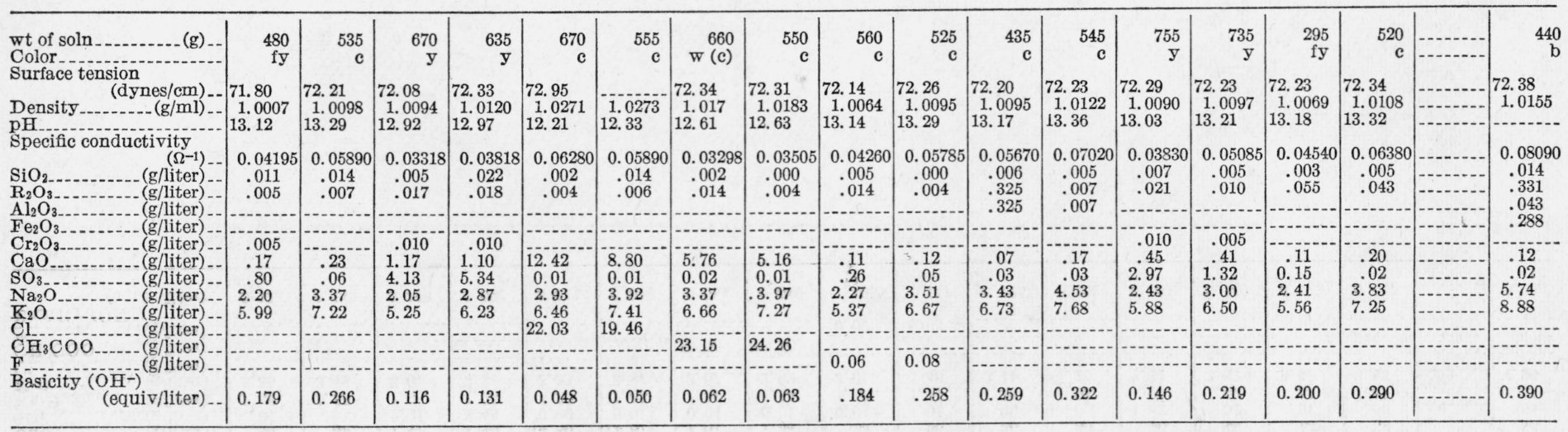

CLINKER 7

\begin{tabular}{|c|c|c|c|c|c|c|c|c|c|c|c|c|c|c|c|c|c|c|}
\hline $\begin{array}{l}\text { wt of soln } \\
\text { Color }\end{array}$ & $\begin{array}{r}330 \\
\mathbf{c}\end{array}$ & $\begin{array}{r}350 \\
\mathrm{c}\end{array}$ & $\begin{array}{r}525 \\
\text { fy }\end{array}$ & $\begin{array}{r}470 \\
\text { fy }\end{array}$ & $\begin{array}{r}610 \\
\mathrm{c}\end{array}$ & $\begin{array}{r}325 \\
\mathrm{c}\end{array}$ & $\begin{array}{r}365 \\
\mathrm{w}(\mathrm{c})\end{array}$ & $\begin{array}{r}115 \\
\mathrm{c}\end{array}$ & $\begin{array}{r}235 \\
\mathrm{c}\end{array}$ & $\begin{array}{r}355 \\
\mathrm{c}\end{array}$ & $\begin{aligned} & 330 \\
& \mathrm{c}\end{aligned}$ & $\begin{array}{r}375 \\
\mathrm{c}\end{array}$ & $\begin{array}{r}365 \\
\mathrm{c}\end{array}$ & $\begin{aligned} 995 \\
\mathrm{c}\end{aligned}$ & $\begin{array}{r}345 \\
\mathrm{c}\end{array}$ & $\begin{array}{r}385 \\
\mathrm{c}\end{array}$ & & \\
\hline nsion & 7200 & 7204 & 72,00 & 7200 & 7288 & 72.46 & 72.25 & & & & & & & 72 & 17. & & & \\
\hline $\begin{array}{l}\text { Density }-. . .(\mathrm{g} / \mathrm{ml})_{-.} \\
\text {pH }\end{array}$ & $\begin{array}{l}2.00 \\
13.0027\end{array}$ & $\begin{array}{l}1.04 \\
13.16\end{array}$ & $\begin{array}{l}2.00 \\
12.0044\end{array}$ & $\begin{array}{l}1.0046 \\
12.79\end{array}$ & $\begin{array}{l}2.08 \\
1.0250 \\
12.17\end{array}$ & $\begin{array}{l}1.0176 \\
12.19\end{array}$ & $\begin{array}{l}1.0113 \\
12.47\end{array}$ & $\begin{array}{l}1.0052 \\
12.65\end{array}$ & $\begin{array}{l}1.0017 \\
12.88\end{array}$ & $\mid \begin{array}{l}2.0035 \\
13.00\end{array}$ & $\begin{array}{l}1.0032 \\
12.99\end{array}$ & $\begin{array}{l}1.0042 \\
13.05\end{array}$ & $\begin{array}{l}\text { 1. } 0023 \\
12.91\end{array}$ & $\begin{array}{l}1.0034 \\
13.00\end{array}$ & $\begin{array}{l}1.9027 \\
12.95\end{array}$ & $\begin{array}{l}1.0038 \\
13.03\end{array}$ & & \\
\hline $\begin{array}{l}\text { pecific conductivity } \\
\left(\Omega^{-1}\right) \\
(\mathrm{g} / \mathrm{liter})\end{array}$ & $\begin{array}{l}0.02660 \\
.001\end{array}$ & $\begin{array}{l}0.03050 \\
.002\end{array}$ & $\begin{array}{c}0.02139 \\
.001\end{array}$ & $\begin{array}{l}0.02156 \\
.001\end{array}$ & $\begin{array}{l}0.05610 \\
.000\end{array}$ & $\begin{array}{l}0.04400 \\
.000\end{array}$ & $\begin{array}{l}0.02314 \\
.005\end{array}$ & $\begin{array}{l}0.02092 \\
.007\end{array}$ & $\begin{array}{l}0.02245 \\
.001\end{array}$ & $\begin{array}{l}0.03080 \\
.002\end{array}$ & $\begin{array}{l}0.02872 \\
.003\end{array}$ & $\begin{array}{l}0.03377 \\
.001\end{array}$ & $\begin{array}{l}0.02433 \\
.002\end{array}$ & $\begin{array}{l}0.02981 \\
.005\end{array}$ & $\begin{array}{l}0.02666 \\
.004\end{array}$ & $\begin{array}{l}0.03192 \\
.005\end{array}$ & & \\
\hline $\begin{array}{l}\mathrm{R}_{2} \mathrm{O}_{3} \\
\mathrm{CaO}\end{array}$ & .003 & .003 & .006 & $\begin{array}{r}.002 \\
1.44\end{array}$ & $\begin{array}{r}.006 \\
15.17\end{array}$ & $\begin{array}{l}.001 \\
9.80\end{array}$ & $\begin{array}{r}.007 \\
6.02\end{array}$ & .027 & .016 & $\begin{array}{l}.005 \\
.27\end{array}$ & .017 & $\begin{array}{l}.003 \\
32\end{array}$ & $\begin{array}{l}.014 \\
.57\end{array}$ & $\begin{array}{l}.009 \\
.47\end{array}$ & $\begin{array}{l}.018 \\
32\end{array}$ & $\begin{array}{l}.005 \\
.36\end{array}$ & & \\
\hline -(g/liter) & .02 & .00 & $\begin{array}{l}1.57 \\
1.91\end{array}$ & $\begin{array}{l}1.44 \\
2.03\end{array}$ & $\begin{array}{r}15.17 \\
0.00\end{array}$ & $\begin{array}{l}9.8 \\
0.0\end{array}$ & $\begin{array}{l}0.02 \\
0.02\end{array}$ & & .02 (.2) & .01 & .00 & .00 & & .47 & .00 & .00 & & \\
\hline - (g/liter) -. & 1. 26 & 1. 57 & 0.87 & 0.97 & .98 & 1.38 & 1.29 & 2.15 & 1. 13 & 1.74 & 1.58 & 1.96 & 1.05 & 1.54 & 1.41 & 1.79 & & \\
\hline $\begin{array}{l}\mathrm{K}_{2} \mathrm{O} \\
\mathrm{Cl}\end{array}$ & 2.84 & 3.20 & 2.2 & 2 & $\begin{array}{r}2.59 \\
20.30\end{array}$ & $\begin{array}{r}3.14 \\
14.69\end{array}$ & & 3.8 & 2.46 & 3.34 & 3.17 & 3.84 & 2. 63 & 3.25 & 2.99 & 3. 22 & & \\
\hline $\mathrm{CH}_{3} \mathrm{COO}_{-}$(g/liter) & & - & & $\ldots$ & & & 15.39 & 7.07 & & & & & & & & & & \\
\hline (g/liter) & & & & & & & & & 0.02 & 0.02 & & & & & & & & \\
\hline Basicity $\left(\mathrm{OH}^{-} \mathrm{H}^{-}\right.$(equiv/liter) & 0.117 & 0.136 & 0.085 & 0.084 & 0.052 & 0.042 & 0.052 & 0.056 & .097 & .135 & 0.126 & 0.150 & 0.109 & 0.133 & 0.118 & 0.143 & & \\
\hline
\end{tabular}


TABLE 3.-Chemical composition and physical properties of the aqueous phase of Portland cement clinker pastes with and without added materials-Continued

\begin{tabular}{|c|c|c|c|c|c|c|c|c|c|c|c|c|c|c|c|c|c|c|}
\hline \multirow{3}{*}{ Added material. } & \multicolumn{2}{|c|}{ None } & \multicolumn{2}{|c|}{ Gypsum } & \multicolumn{2}{|c|}{$\begin{array}{l}\text { Calcium } \\
\text { chloride }\end{array}$} & \multicolumn{2}{|c|}{$\begin{array}{l}\text { Calcium } \\
\text { acetate }\end{array}$} & \multicolumn{2}{|c|}{ Fluosilicic acid } & \multicolumn{2}{|c|}{ Sucrose } & \multicolumn{2}{|c|}{ T D A } & \multicolumn{2}{|c|}{ Tannic acid } & \multicolumn{2}{|c|}{$\begin{array}{l}\text { Tri- } \\
\text { ethanolamine }\end{array}$} \\
\hline & $7 \mathrm{~min}$ & $2 \mathrm{hr}$ & $7 \mathrm{~min}$ & $2 \mathrm{hr}$ & $7 \mathrm{~min}$ & $2 \mathrm{hr}$ & $7 \mathrm{~min}$ & $2 \mathrm{hr}$ & $7 \mathrm{~min}$ & $2 \mathrm{hr}$ & $7 \mathrm{~min}$ & $2 \mathrm{hr}$ & $7 \mathrm{~min}$ & $2 \mathrm{hr}$ & $7 \mathrm{~min}$ & $2 \mathrm{hr}$ & $7 \min$ & $2 \mathrm{hr}$ \\
\hline & \multicolumn{18}{|c|}{ CLINKER 8} \\
\hline $\begin{array}{l}\text { wt of soln } \\
\text { Color }\end{array}$ & $\begin{array}{r}510 \\
\mathrm{c}\end{array}$ & $\begin{array}{r}560 \\
\mathrm{c}\end{array}$ & $\begin{array}{r}640 \\
\mathrm{y}\end{array}$ & $\begin{array}{r}620 \\
\mathrm{y}\end{array}$ & $\begin{array}{r}670 \\
\mathrm{c}\end{array}$ & $\begin{array}{r}500 \\
\mathrm{c}\end{array}$ & $\begin{array}{r}405 \\
\mathrm{w}(\mathrm{c})\end{array}$ & $\begin{array}{r}385 \\
\mathrm{c}\end{array}$ & $\begin{array}{r}385 \\
\mathrm{c}\end{array}$ & $\begin{array}{r}595 \\
\mathrm{c}\end{array}$ & $\begin{array}{r}445 \\
\mathrm{c}\end{array}$ & $\begin{array}{r}540 \\
\mathrm{c}\end{array}$ & $\begin{array}{r}715 \\
\text { fy }\end{array}$ & $\begin{array}{r}590 \\
\text { fy }\end{array}$ & $\begin{array}{r}325 \\
\mathrm{c}\end{array}$ & $\begin{array}{r}485 \\
\mathrm{c}\end{array}$ & & 215 \\
\hline $\begin{array}{l}\text { Surface tension } \\
\text { Density }(\text { dynes } / \mathrm{cm}) \\
\quad-\end{array}$ & $\begin{array}{l}72.00 \\
1.0035\end{array}$ & $\begin{array}{l}\text { 72. } 01 \\
1.0040\end{array}$ & $\mid$\begin{tabular}{|l|}
72.17 \\
1.0052
\end{tabular} & \begin{tabular}{|l|}
72.11 \\
1.0057
\end{tabular} & \begin{tabular}{|l|}
72.99 \\
1.0259
\end{tabular} & \begin{tabular}{|l|}
72.80 \\
1.0234
\end{tabular} & \begin{tabular}{|l|}
72.30 \\
1.0151
\end{tabular} & $\begin{array}{l}72.19 \\
1.0150\end{array}$ & $\begin{array}{l}71.95 \\
1.0022\end{array}$ & $\begin{array}{l}72.13 \\
1.0041\end{array}$ & $\begin{array}{l}71.98 \\
1.0030\end{array}$ & $\begin{array}{l}72.19 \\
1.0046\end{array}$ & $\begin{array}{l}72.05 \\
1.0042\end{array}$ & $\begin{array}{l}72.10 \\
1.0037\end{array}$ & \begin{tabular}{|l|}
71.97 \\
1.0032
\end{tabular} & $\begin{array}{l}72.08 \\
1.0041\end{array}$ & & $\begin{array}{l}71.9 \\
1.0066\end{array}$ \\
\hline $\begin{array}{l}\text { pH } \\
\text { Specific conductivity }\end{array}$ & 13.01 & 13.05 & 12.82 & 12.81 & 12.16 & 12.19 & 12.46 & 12.43 & 12.91 & 13.04 & 12.98 & 13. 09 & & 13.02 & 12.97 & 13.02 & & \\
\hline $\begin{array}{l}\left(\Omega^{-1}\right) \\
\mathrm{SiO} \\
\mathrm{R}_{2} \mathrm{O}_{3} \\
\mathrm{Al}_{2} \mathrm{O}_{3}\end{array}$ & $\begin{array}{l}0.03061 \\
.002 \\
.006\end{array}$ & $\begin{array}{l}0.03306 \\
.002 \\
.006\end{array}$ & $\begin{array}{l}0.02394 \\
.002 \\
.003\end{array}$ & $\begin{array}{c}0.02504 \\
.003 \\
.004\end{array}$ & $\begin{array}{l}0.05815 \\
.000 \\
.010\end{array}$ & \begin{tabular}{|l}
0.05445 \\
.000 \\
.008
\end{tabular} & $\begin{array}{l}0.02655 \\
.003 \\
.027\end{array}$ & \begin{tabular}{|l|}
0.02655 \\
.001 \\
.005
\end{tabular} & $\begin{array}{l}0.02441 \\
.001 \\
.009\end{array}$ & $\begin{array}{l}0.03320 \\
.001 \\
.003\end{array}$ & \begin{tabular}{|c|}
0.02843 \\
.002 \\
.033 \\
.033
\end{tabular} & $\begin{array}{l}0.03591 \\
.002 \\
.008\end{array}$ & $\begin{array}{l}0.02162 \\
.001 \\
.015\end{array}$ & $\begin{array}{l}0.03146 \\
.004 \\
.009\end{array}$ & $\begin{array}{l}0.02930 \\
.003 \\
.016\end{array}$ & $\begin{array}{l}0.03302 \\
.003 \\
.002\end{array}$ & & $\begin{array}{l}0.04322 \\
.002 \\
.015\end{array}$ \\
\hline . & .44 & .39 & 1.47 & 1.40 & 14.86 & 12.87 & $7 . \overline{6}$ & 7.38 & .23 & .36 & .36 & .36 & .9 & $\cdots$ & .23 & .38 & & .25 \\
\hline --(g/liter) -- & .01 & .01 & 2. 21 & 2.48 & 0.00 & 0.01 & 0.04 & 0.01 & 0.01 & .01 & .00 & .00 & 1.83 & .02 & .01 & .00 & & .01 \\
\hline .. (g/liter) -. & 1.46 & 1.65 & 1.08 & 1. 25 & 1.39 & 1. 62 & 1. & 1.74 & 1.13 & 1.71 & 1.38 & 1.93 & 1. 01 & 1.49 & 1.52 & 1.69 & & 2.55 \\
\hline $\begin{array}{l}\mathrm{K}_{2} \mathrm{O} \\
\mathrm{Cl}\end{array}$ & 3.35 & 3.48 & 3.07 & 3.34 & $\begin{array}{r}3.33 \\
21.27\end{array}$ & $\begin{array}{r}3.87 \\
19.23\end{array}$ & 3. & 3.92 & 3. 01 & 3.4 & 3.11 & 3.76 & 3. & 3. 62 & 3.49 & 3.83 & & 4. 96 \\
\hline $\mathrm{CH}_{3} \mathrm{COO}_{-}$(g/liter) & & & & & & & 20.20 & 20.86 & & & & & & & & & & \\
\hline F & & & & & & & & & 0.02 & 0.03 & & & & & & & & \\
\hline $\begin{array}{l}\text { Basicity (equiv/liter) } \\
\text { (OH }\end{array}$ & 0.136 & 0.147 & 0.093 & 0.095 & 0.047 & 0.048 & 0.054 & 0.049 & .105 & .147 & 0.125 & 0.161 & 0.081 & 0.140 & 0.129 & 0.147 & & 0.205 \\
\hline
\end{tabular}


CLINKER 9

\begin{tabular}{|c|c|c|c|c|c|c|c|c|c|c|c|c|c|c|c|c|c|c|}
\hline $\begin{array}{l}\text { wt of soln } \\
\text { Color }\end{array}$ & $\begin{array}{r}475 \\
\mathrm{c}\end{array}$ & $\begin{array}{r}665 \\
\mathrm{c}\end{array}$ & $\begin{array}{r}585 \\
\mathbf{y}\end{array}$ & $\begin{array}{r}630 \\
\mathbf{y}\end{array}$ & $\begin{array}{r}650 \\
\mathrm{c}\end{array}$ & $\begin{array}{r}480 \\
\mathrm{c}\end{array}$ & $\begin{array}{r}340 \\
\mathrm{c}\end{array}$ & $\begin{array}{r}525 \\
\mathrm{c}\end{array}$ & $\begin{array}{r}565 \\
\text { fy }\end{array}$ & $\begin{array}{r}565 \\
\mathrm{c}\end{array}$ & $\begin{array}{r}535 \\
\text { fy }\end{array}$ & $\begin{array}{r}620 \\
\mathrm{c}\end{array}$ & $\begin{array}{r}840 \\
\mathbf{y}\end{array}$ & $\begin{array}{r}710 \\
\text { fy }\end{array}$ & $\begin{array}{r}705 \\
\mathrm{y}\end{array}$ & $\begin{array}{r}540 \\
\mathrm{c}\end{array}$ & $\begin{array}{r}125 \\
\mathrm{c}\end{array}$ & $\begin{aligned} 230 \\
b\end{aligned}$ \\
\hline $\begin{array}{l}\text { Surface tension } \\
\text { Density }(\mathrm{dyn} / \mathrm{cm}) \\
\text { pH }\end{array}$ & $\begin{array}{l}72.13 \\
1.0030 \\
12.98\end{array}$ & $\mid \begin{array}{l}72.24 \\
1.0037 \\
13.03\end{array}$ & \begin{tabular}{|l|}
72.18 \\
1.0045 \\
12.78
\end{tabular} & \begin{tabular}{|l}
72.16 \\
1.0056 \\
12.80
\end{tabular} & $\mid$\begin{tabular}{|l}
72.96 \\
1.0248 \\
12.20
\end{tabular} & \begin{tabular}{|l|}
72.83 \\
1.0228 \\
12.20
\end{tabular} & \begin{tabular}{|l}
72.43 \\
1.0156 \\
12.65
\end{tabular} & \begin{tabular}{|l}
72.38 \\
1.0150 \\
12.66
\end{tabular} & $\begin{array}{l}1.0012 \\
12.73\end{array}$ & \begin{tabular}{|l|}
72.26 \\
1.0037 \\
13.01
\end{tabular} & \begin{tabular}{|l}
72.13 \\
1.0021 \\
12.91
\end{tabular} & \begin{tabular}{|l|}
72.31 \\
1.0038 \\
13.04
\end{tabular} & \begin{tabular}{|l}
72.00 \\
1.0036 \\
12.78
\end{tabular} & $\mid \begin{array}{l}72.27 \\
1.0032 \\
12.92\end{array}$ & \begin{tabular}{|l}
72.10 \\
1.0023 \\
12.82
\end{tabular} & \begin{tabular}{|l} 
72. 20 \\
1.0037 \\
13.03
\end{tabular} & \begin{tabular}{|l|}
71.95 \\
1.0032
\end{tabular} & $\begin{array}{l}72.44 \\
1.0105\end{array}$ \\
\hline $\begin{array}{l}\text { Specific conductivity } \\
(\Omega-1) \\
\mathrm{SiO}_{2} \\
\mathrm{R}_{2} \mathrm{O}_{3-} \\
\mathrm{Cr}_{2} \mathrm{O}_{3}\end{array}$ & $\begin{array}{l}0.02762 \\
.000 \\
.003\end{array}$ & $\begin{array}{l}0.03158 \\
.001 \\
.001\end{array}$ & $\begin{array}{l}0.02148 \\
.001 \\
.008 \\
.005\end{array}$ & $\begin{array}{l}0.02390 \\
.002 \\
.009 \\
.005\end{array}$ & $\begin{array}{l}0.05595 \\
.001 \\
.006\end{array}$ & $\begin{array}{l}0.05280 \\
.002 \\
.008\end{array}$ & $\begin{array}{l}0.02600 \\
.002 \\
.033\end{array}$ & $\begin{array}{l}0.02630 \\
.000 \\
.011\end{array}$ & $\begin{array}{l}0.01759 \\
.001 \\
.007\end{array}$ & $\begin{array}{l}0.03060 \\
.002 \\
.004\end{array}$ & $\begin{array}{l}0.02330 \\
.003 \\
.011\end{array}$ & $\begin{array}{l}0.03178 \\
.003 \\
.004\end{array}$ & $\begin{array}{l}0.02060 \\
.002 \\
.016\end{array}$ & $\begin{array}{l}0.02693 \\
.002 \\
.009\end{array}$ & $\begin{array}{l}12.02 \\
0.02053 \\
.003 \\
.007 \\
.005\end{array}$ & $\begin{array}{c}10.00 \\
0.03139 \\
.004 \\
.002\end{array}$ & $\begin{array}{l}0.02497 \\
.002 \\
.593\end{array}$ & $\begin{array}{l}0.06040 \\
.005 \\
.088\end{array}$ \\
\hline 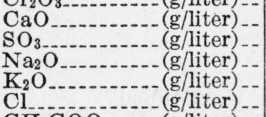 & $\begin{array}{r}.80 \\
.00 \\
2.99 \\
0.35\end{array}$ & $\begin{array}{r}.43 \\
.00 \\
3.89 \\
0.35\end{array}$ & $\begin{array}{l}.000 \\
1.44 \\
2.08 \\
2.53 \\
0.28\end{array}$ & $\begin{array}{l}\text { 1. } 31 \\
2.55 \\
3.27 \\
0.33\end{array}$ & $\begin{array}{r}14.21 \\
0.01 \\
3.94 \\
0.33 \\
20.70\end{array}$ & $\begin{array}{r}12.15 \\
0.01 \\
4.77 \\
0.34 \\
19.25\end{array}$ & $\begin{array}{l}8.27 \\
0.03 \\
3.85 \\
0.30 \\
-\end{array}$ & $\begin{array}{r}7.07 \\
0.00 \\
4.82 \\
0.29 \\
-\end{array}$ & $\begin{array}{r}.50 \\
.31 \\
2.11 \\
0.21\end{array}$ & $\begin{array}{l}.52 \\
.01 \\
3.84 \\
0.44\end{array}$ & $\begin{array}{r}.58 \\
.08 \\
2.71 \\
0.34\end{array}$ & $\begin{array}{r}.45 \\
.01 \\
3.96 \\
0.31\end{array}$ & $\begin{array}{l}1.24 \\
1.55 \\
2.55 \\
0.26\end{array}$ & $\begin{array}{r}.85 \\
.16 \\
3.06 \\
0.31\end{array}$ & $\begin{array}{l}.000 \\
.69 \\
.68 \\
2.50 \\
0.25\end{array}$ & $\begin{array}{r}.39 \\
.01 \\
4.00 \\
0.31\end{array}$ & $\begin{array}{r}.28 \\
.01 \\
3.45 \\
0.38\end{array}$ & $\begin{array}{r}.14 \\
.01 \\
8.83 \\
0.60\end{array}$ \\
\hline $\mathrm{CH}_{3} \mathrm{COO}$ & $\cdots$ & 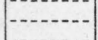 & 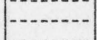 & & & & 21.40 & 21. 11 & 03 & & & & & & & & & \\
\hline $\begin{array}{r}\text { Basicity } \\
\text { (equiv/liter) }\end{array}$ & .133 & .150 & .089 & .096 & 0.050 & 0.047 & 0.059 & 0.056 & .079 & .145 & .109 & .151 & .088 & .128 & .092 & .147 & .13 & .31 \\
\hline \multicolumn{19}{|c|}{ CLINKER 10} \\
\hline $\begin{array}{l}\text { wt of soln } \\
\text { Color } \\
\text { Surface tension }\end{array}$ & $\begin{array}{r}605 \\
\mathrm{c}\end{array}$ & 695 & $\begin{array}{r}700 \\
\text { fy }\end{array}$ & $\begin{array}{c}665 \\
\text { vfy }\end{array}$ & $\begin{array}{r}795 \\
\mathrm{c}\end{array}$ & $\begin{array}{r}555 \\
\mathrm{c}\end{array}$ & $\begin{array}{r}495 \\
\mathrm{w}(\mathrm{c})\end{array}$ & $\begin{array}{r}460 \\
\mathrm{w}(\mathrm{c})\end{array}$ & 515 & $\begin{array}{r}640 \\
\mathrm{c}\end{array}$ & $\begin{array}{r}525 \\
\mathrm{c}\end{array}$ & $\begin{array}{r}605 \\
\mathrm{c}\end{array}$ & $\begin{array}{r}905 \\
\text { fy }\end{array}$ & $\begin{array}{r}730 \\
\mathrm{c}\end{array}$ & $\begin{array}{r}450 \\
\mathrm{c}\end{array}$ & $\begin{array}{r}635 \\
\mathrm{c}\end{array}$ & $\begin{array}{l}350 \\
\nabla f b\end{array}$ & $\begin{array}{r}485 \\
\mathrm{fb}\end{array}$ \\
\hline $\begin{array}{l}\text { (dynes/cm) } \\
\text { Density } \\
\text { pH }\end{array}$ & $\begin{array}{l}\text { 71. } 99 \\
1.0011 \\
12.81\end{array}$ & \begin{tabular}{|l}
72.21 \\
1.0011 \\
12.80
\end{tabular} & $\begin{array}{l}72.11 \\
1.0028 \\
12.72\end{array}$ & \begin{tabular}{|l} 
72. 21 \\
1.0028 \\
12.71
\end{tabular} & \begin{tabular}{|l|}
73.16 \\
1.0280 \\
12.15
\end{tabular} & \begin{tabular}{|l|}
73.00 \\
1.0260 \\
12.14
\end{tabular} & $\begin{array}{l}1.0185 \\
12.61\end{array}$ & 1.0170 & \begin{tabular}{|l|}
71.96 \\
1.0008 \\
12.85
\end{tabular} & \begin{tabular}{|l}
72.03 \\
1.0008 \\
12.86
\end{tabular} & \begin{tabular}{|l}
72.25 \\
1.0014 \\
12.82
\end{tabular} & \begin{tabular}{|l}
72.16 \\
1.0012 \\
12.79
\end{tabular} & \begin{tabular}{|l|}
72.16 \\
1.0012 \\
12.71
\end{tabular} & $\begin{array}{l}72.17 \\
1.0011 \\
12.76\end{array}$ & $\begin{array}{l}71.96 \\
1.0011 \\
12.80\end{array}$ & $\begin{array}{l}72.10 \\
1.0010 \\
12.80\end{array}$ & $\begin{array}{l}71.96 \\
1.0018\end{array}$ & $\begin{array}{l}71.97 \\
1.0023\end{array}$ \\
\hline $\begin{array}{l}\begin{array}{l}\text { Specific conductivity } \\
(\Omega-1)\end{array} \\
\mathrm{SiO}^{-1} \\
\mathrm{R}_{2} \mathrm{O}_{3} \\
\mathrm{Al}_{2} \mathrm{O}_{3} \\
\mathrm{Fe}_{2} \mathrm{O}_{3}\end{array}$ & $\begin{array}{l}0.01783 \\
.001 \\
.005\end{array}$ & $\begin{array}{l}8.01773 \\
.003 \\
.013\end{array}$ & $\begin{array}{l}0.01705 \\
.003 \\
.005\end{array}$ & $\begin{array}{l}0.01678 \\
.003 \\
.004\end{array}$ & $\begin{array}{l}0.06000 \\
.002 \\
.011\end{array}$ & $\begin{array}{l}0.05675 \\
.003 \\
.011\end{array}$ & \begin{tabular}{|l}
0.02539 \\
.151 \\
.494 \\
.417 \\
.077
\end{tabular} & $\begin{array}{l}0.02467 \\
.070 \\
.232 \\
.216 \\
.016\end{array}$ & $\begin{array}{l}0.01598 \\
.001 \\
.003\end{array}$ & $\begin{array}{l}0.01656 \\
.002 \\
.002\end{array}$ & $\begin{array}{c}0.01882 \\
.002 \\
.003\end{array}$ & $\begin{array}{c}0.01824 \\
.002 \\
.002\end{array}$ & $\begin{array}{l}0.01557 \\
.002 \\
.004\end{array}$ & $\begin{array}{c}0.01717 \\
.002 \\
.003\end{array}$ & $\begin{array}{l}0.01771 \\
.002 \\
.003\end{array}$ & $\begin{array}{l}0.01758 \\
.001 \\
.000\end{array}$ & $\begin{array}{l}0.01778 \\
.005 \\
.274 \\
.014 \\
.260\end{array}$ & $\begin{array}{l}0.02178 \\
.003 \\
.083 \\
.003 \\
.080\end{array}$ \\
\hline.- (g/liter)-- & 1.28 & 1.03 & 2.03 & 1.87 & 18.28 & 16.69 & 12.43 & 11.06 & 1.35 & 1.00 & 1.50 & 1. 03 & 1. 41 & 1. 21 & 1. 33 & 1.03 & $\begin{array}{l}.260 \\
1.38\end{array}$ & $\begin{array}{r}.080 \\
1.04\end{array}$ \\
\hline $\begin{array}{l}\text { (g/liter) }-- \\
\text { (g/liter) -- }\end{array}$ & 0.01 & 0.02 & 1. 29 & 1.37 & 0.00 & 0.00 & 0.24 & 0.13 & 0.00 & 0.00 & 0.01 & 0.01 & & & 0.01 & & 0.00 & 0.00 \\
\hline $\begin{array}{l}\mathrm{K}_{2} \mathrm{O} \\
\mathrm{C} 1\end{array}$ & 1. 45 & 1.83 & & 1. 63 & $\begin{array}{r}.00 \\
22.81\end{array}$ & $\begin{array}{r}.70 \\
21.27\end{array}$ & 1.70 & 190 & & 1. 65 & 1. 59 & 1.8 & 1. & 1. 61 & 1. & 1.74 & 1.66 & 2. .38 \\
\hline $\mathrm{CH}_{3} \mathrm{COO}_{-}$(g/liter) & & - & & & & & 21.70 & 21. 34 & & & & & & & & & & \\
\hline $\begin{array}{c}\text { (equiv/liter) } \\
\text { Basicity }\end{array}$ & 0.085 & 0.082 & 0.076 & 0.072 & 0.054 & 0.050 & 0.090 & 0.060 & $\begin{array}{l}0.00 \\
.077\end{array}$ & $\begin{array}{l}0.01 \\
.076\end{array}$ & 0.091 & 0.085 & 0.073 & 0.082 & 0.085 & 0.082 & 0.096 & 0.112 \\
\hline
\end{tabular}


TABLE 3.-Chemical composition and physical properties of the aqueous phase of Portland cement clinker pastes with and without added materials-Continued

\begin{tabular}{|c|c|c|c|c|c|c|c|c|c|c|c|c|c|c|c|c|c|c|}
\hline \multirow{3}{*}{ Added material. } & \multicolumn{2}{|c|}{ None } & \multicolumn{2}{|c|}{ Gypsum } & \multicolumn{2}{|c|}{$\begin{array}{l}\text { Calcium } \\
\text { chloride }\end{array}$} & \multicolumn{2}{|c|}{$\begin{array}{l}\text { Calcium } \\
\text { acetate }\end{array}$} & \multicolumn{2}{|c|}{ Fluosilicic acid } & \multicolumn{2}{|c|}{ Sucrose } & \multicolumn{2}{|c|}{ T D A } & \multicolumn{2}{|c|}{ Tannic acid } & \multicolumn{2}{|c|}{$\begin{array}{l}\text { Tri- } \\
\text { ethanolamine }\end{array}$} \\
\hline & $7 \min$ & $2 \mathrm{hr}$ & $7 \mathrm{~min}$ & $2 \mathrm{hr}$ & $7 \mathrm{~min}$ & $2 \mathrm{hr}$ & $7 \mathrm{~min}$ & $2 \mathrm{hr}$ & $7 \mathrm{~min}$ & $2 \mathrm{hr}$ & $7 \mathrm{~min}$ & $2 \mathrm{hr}$ & $i \min$ & $2 \mathrm{hr}$ & $\min$ & $2 \mathrm{hr}$ & $7 \mathrm{~min}$ & $2 \mathrm{hr}$ \\
\hline & \multicolumn{18}{|c|}{ CLINKER 11} \\
\hline $\begin{array}{l}\text { wt. of soln } \\
\text { Color } \\
\text { Surface tension }\end{array}$ & $\begin{array}{r}405 \\
\mathrm{c}\end{array}$ & $\begin{array}{r}345 \\
\mathrm{c}\end{array}$ & $\begin{array}{r}585 \\
\text { fy }\end{array}$ & $\begin{array}{r}550 \\
\text { fy }\end{array}$ & $\begin{array}{r}575 \\
\mathrm{c}\end{array}$ & $\begin{array}{r}310 \\
\mathrm{c}\end{array}$ & $\begin{array}{r}325 \\
\mathrm{w}(\mathrm{c})\end{array}$ & $\begin{array}{r}400 \\
c\end{array}$ & $\begin{array}{r}270 \\
\mathrm{c}\end{array}$ & $\begin{array}{r}435 \\
\mathrm{c}\end{array}$ & $\begin{array}{r}340 \\
\mathrm{c}\end{array}$ & $\begin{array}{r}365 \\
\mathrm{c}\end{array}$ & $\begin{array}{r}540 \\
\mathrm{c}\end{array}$ & $\begin{array}{l}535 \\
\text { vfy }\end{array}$ & $\begin{array}{r}390 \\
\mathrm{c}\end{array}$ & $\begin{array}{r}330 \\
\mathrm{c}\end{array}$ & $\begin{array}{r}220 \\
\mathrm{~b}\end{array}$ & $\begin{array}{l}145 \\
b\end{array}$ \\
\hline $\begin{array}{l}\text { Density }(\text { dynes/cm })-- \\
\text { pH }\end{array}$ & \begin{tabular}{|l|}
72.18 \\
1.0037 \\
13.02
\end{tabular} & $\begin{array}{l}72.15 \\
1.0042 \\
13.03\end{array}$ & \begin{tabular}{|l}
72.13 \\
1.0045 \\
12.80
\end{tabular} & $\begin{array}{l}72.18 \\
1.0045 \\
12.79\end{array}$ & $\begin{array}{l}73.00 \\
1.0259 \\
12.20\end{array}$ & $\begin{array}{l}72.85 \\
1.0197 \\
12.17\end{array}$ & $\begin{array}{c}72.41 \\
1.0139 \\
12.50\end{array}$ & \begin{tabular}{|l|}
72.43 \\
1.0123 \\
12.48
\end{tabular} & $\begin{array}{l}72.15 \\
1.0025 \\
12.91\end{array}$ & $\begin{array}{l}72.31 \\
1.0043 \\
13.04\end{array}$ & \begin{tabular}{|l}
72.33 \\
1.0044 \\
13.06
\end{tabular} & $\begin{array}{l}72.48 \\
1.0052 \\
13.06\end{array}$ & $\begin{array}{l}72.21 \\
1.0024 \\
12.89\end{array}$ & $\mid \begin{array}{l}72.20 \\
1.0038 \\
12.99\end{array}$ & $\begin{array}{l}72.28 \\
1.0034 \\
12.96\end{array}$ & $\begin{array}{l}71.91 \\
1.0043 \\
13.02\end{array}$ & $\begin{array}{l}71.91 \\
1.0063\end{array}$ & $\begin{array}{l}71.75 \\
1.0068\end{array}$ \\
\hline Specific conductivity & 0003131 & & & & & & & & & & & & & & & & & \\
\hline 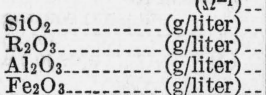 & $\begin{array}{l}.0 .006 \\
.004 \\
.004\end{array}$ & $\begin{array}{l}0.03358 \\
.001 \\
.001\end{array}$ & $\begin{array}{c}0.02202 \\
.003 \\
.006\end{array}$ & $\begin{array}{c}0.02189 \\
.003 \\
.007 \\
-0\end{array}$ & $\left|\begin{array}{l}0.05760 \\
.005 \\
.003\end{array}\right|$ & $\begin{array}{l}0.04762 \\
.004 \\
.005 \\
-0.0\end{array}$ & $\begin{array}{l}0.02562 \\
.001 \\
.009 \\
\end{array}$ & $\begin{array}{c}0.02458 \\
.000 \\
.006 \\
-0 .-\end{array}$ & $\begin{array}{c}0.02559 \\
.007 \\
.023 \\
-0--\end{array}$ & $\begin{array}{l}0.03469 \\
.003 \\
.004\end{array}$ & $\begin{array}{c}0.03466 \\
.002 \\
.025 \\
\end{array}$ & $\begin{array}{c}0.03829 \\
.003 \\
.007\end{array}$ & $\mid$\begin{tabular}{c}
0.02436 \\
.003 \\
.007 \\
\hdashline-
\end{tabular} & $\mid$\begin{tabular}{c}
0.03121 \\
.002 \\
.009 \\
\hdashline-
\end{tabular} & $\begin{array}{l}0.02997 \\
.013 \\
.015\end{array}$ & $\begin{array}{l}0.03372 \\
.004 \\
.004 \\
\end{array}$ & $\begin{array}{l}0.03911 \\
.025 \\
.340 \\
.055\end{array}$ & $\begin{array}{l}0.04166 \\
.020 \\
.248 \\
.022\end{array}$ \\
\hline $\begin{array}{l}\mathrm{Fe}_{2} \mathrm{O}_{3} \\
\mathrm{CaO}\end{array}$ & $.40^{-}$ & .39 & 1.75 & 1.47 & 15.51 & 10.66 & 7.39 & 6.68 & .19 & .30 & .26 & .35 & .77 & .54 & .48 & .31 & $\begin{array}{l}.285 \\
.10\end{array}$ & $\begin{array}{l}.226 \\
.09\end{array}$ \\
\hline $\mathrm{SO}_{3}$ & .02 & .01 & 1.82 & 2.13 & 0.01 & 0.02 & 0.01 & 0.01 & .01 & .00 & .00 & .00 & .03 & .00 & .01 & .01 & .01 & .01 \\
\hline $\mathrm{Na}_{2} \mathrm{O}$ & 1.78 & 1.97 & 0.77 & 0.99 & 1.11 & 1.80 & 1.48 & 1.91 & 1.49 & 2.20 & 2.15 & 2.41 & .95 & 1.69 & 1. 77 & 2.00 & 2.81 & 3.13 \\
\hline $\begin{array}{l}\mathrm{K}_{2} \mathrm{O} \\
\mathrm{Cl}\end{array}$ & 3.28 & 3.48 & 2.44 & 2.65 & $\begin{array}{r}2.78 \\
21.03\end{array}$ & $\begin{array}{r}3.40 \\
16.40\end{array}$ & 3.1 & 3.53 & 2.82 & 3.61 & 3.63 & 3.91 & 2.62 & 3.24 & 3.23 & 3.47 & 3.97 & 4.27 \\
\hline $\mathrm{CH}_{3} \mathrm{COO}_{\ldots}$ & $-\ldots$ & -. & ...... & $-\ldots-$. & & & 18.06 & 19.07 & & & & & & & . & & $\ldots$ & - \\
\hline F & & & & & & & & & 0.00 & 0.02 & & & & & & & & \\
\hline Basicity $\underset{(\text { (equiv/liter) }}{\left(\mathrm{OH}^{-}\right)}$ & 0.139 & 0.150 & 0.091 & 0.085 & 0.053 & 0.040 & 0.057 & 0.050 & 0.110 & .155 & 0.152 & 0.172 & 0.110 & 0.140 & 0.131 & 0.151 & 0.184 & 0.199 \\
\hline
\end{tabular}


CLINKER 12

\begin{tabular}{|c|c|c|c|c|c|c|c|c|c|c|c|c|c|c|c|c|c|c|}
\hline $\begin{array}{l}\text { wt of soln } \\
\text { Color }\end{array}$ & $\begin{array}{r}315 \\
\mathrm{c}\end{array}$ & $\begin{array}{r}405 \\
\mathrm{c}\end{array}$ & $\begin{array}{r}515 \\
\mathrm{y}\end{array}$ & $\begin{array}{r}515 \\
\mathrm{y}\end{array}$ & $\begin{aligned} 580 \\
\mathrm{c}\end{aligned}$ & $\begin{array}{r}280 \\
\mathrm{c}\end{array}$ & $\begin{array}{r}425 \\
\mathrm{w}(\mathrm{c})\end{array}$ & $\begin{array}{r}370 \\
\mathrm{c}\end{array}$ & $\begin{array}{r}305 \\
\mathrm{c}\end{array}$ & $\begin{array}{r}435 \\
\mathrm{c}\end{array}$ & $\begin{array}{r}285 \\
\mathrm{c}\end{array}$ & $\begin{array}{r}285 \\
\mathrm{c}\end{array}$ & $\begin{array}{r}555 \\
\mathrm{y}\end{array}$ & $\begin{array}{r}445 \\
\text { fy }\end{array}$ & $\begin{array}{r}395 \\
\mathrm{c}\end{array}$ & $\begin{array}{r}290 \\
\text { fy }\end{array}$ & & \\
\hline $\begin{array}{l}\text { Surface tension } \\
\text { Density. } \\
\text { pH }\end{array}$ & $\begin{array}{l}72.24 \\
1.0094 \\
13.26\end{array}$ & \begin{tabular}{|l}
72.06 \\
1.0105 \\
13.30
\end{tabular} & $\begin{array}{l}72.18 \\
1.0112 \\
12.96\end{array}$ & $\begin{array}{l}\text { 71. } 98 \\
1.0119\end{array}$ & $\mid \begin{array}{l}73.10 \\
1.0243 \\
12.30\end{array}$ & \begin{tabular}{|c|}
72.91 \\
1.0211 \\
12.29
\end{tabular} & $\begin{array}{l}72.20 \\
1.0143 \\
12.71\end{array}$ & $\begin{array}{l}72.31 \\
1.0150 \\
12.68\end{array}$ & $\begin{array}{l}72.24 \\
1.0085 \\
13.19\end{array}$ & $\begin{array}{l}72.38 \\
1.0106 \\
13.29\end{array}$ & $\begin{array}{l}72.25 \\
1.0105 \\
13.30\end{array}$ & $\begin{array}{l}72.41 \\
1.0112 \\
13.33\end{array}$ & $\begin{array}{l}72.26 \\
1.0085 \\
\end{array}$ & $\left|\begin{array}{c}72.24 \\
1.0081\end{array}\right|$ & $\begin{array}{l}72.24 \\
1.0095 \\
13.27\end{array}$ & \begin{tabular}{|c|}
72.41 \\
1.0108 \\
13.30
\end{tabular} & & \\
\hline $\begin{array}{l}\text { Specific conductivity } \\
(\Omega-1) \\
\mathrm{SiO}_{2} \\
\mathrm{R}_{2} \mathrm{O}_{3} \\
\mathrm{C}_{3} \text { (g/liter) }\end{array}$ & $-\begin{array}{l}10.20 \\
0.05730 \\
.013 \\
.017\end{array}$ & $\begin{array}{c}0.06240 \\
.011 \\
.006\end{array}$ & $\begin{array}{l}0.03731 \\
.001 \\
.020 \\
.015\end{array}$ & $\begin{array}{l}0.03748 \\
.002 \\
.018 \\
.015\end{array}$ & $\begin{array}{l}0.06005 \\
.002 \\
.007\end{array}$ & $\begin{array}{c}0.04580 \\
.000 \\
.006\end{array}$ & $\begin{array}{l}0.03382 \\
.000 \\
.004\end{array}$ & $\begin{array}{l}0.03513 \\
.002 \\
.002\end{array}$ & $\begin{array}{l}0.05250 \\
.012 \\
.033\end{array}$ & $\begin{array}{l}0.06285 \\
.011 \\
.006\end{array}$ & $\begin{array}{l}10.00 \\
0.06200 \\
.011 \\
.023\end{array}$ & $\begin{array}{c}0.00 \\
0.06590 \\
.010 \\
.012\end{array}$ & $\begin{array}{l}0.03972 \\
.010 \\
.016\end{array}$ & $\begin{array}{l}0.05260 \\
.010 \\
.008\end{array}$ & $\begin{array}{l}15.26 \\
0.06040 \\
.005 \\
.025\end{array}$ & $\begin{array}{c}13.30 \\
0.06340 \\
.013 \\
.018\end{array}$ & & \\
\hline 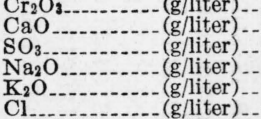 & $\begin{array}{r}.15 \\
.02 \\
10.75\end{array}$ & $\begin{array}{r}.17 \\
.03 \\
.51 \\
11.66\end{array}$ & $\begin{array}{l}.015 \\
1.21 \\
4.68 \\
0.26 \\
8.95\end{array}$ & $\begin{array}{l}.015 \\
1.10 \\
5.11 \\
0.33 \\
9.32\end{array}$ & $\begin{array}{r}10.64 \\
0.01 \\
.28 \\
10.26 \\
19.56\end{array}$ & $\begin{array}{r}7.37 \\
0.01 \\
.43 \\
11.81 \\
17.06\end{array}$ & $\begin{array}{r}3.44 \\
0.01 \\
.46 \\
10.98\end{array}$ & $\begin{array}{r}2.91 \\
0.01 \\
12.65 \\
12.22\end{array}$ & $\begin{array}{r}.07 \\
.03 \\
.28 \\
9.73\end{array}$ & $\begin{array}{r}.14 \\
.01 \\
.48 \\
11.81\end{array}$ & $\begin{array}{r}.12 \\
.02 \\
11.51 \\
11.62\end{array}$ & $\begin{array}{r}.13 \\
.02 \\
.59 \\
12.16\end{array}$ & $\begin{array}{l}.43 \\
2.30 \\
0.14 \\
8.82\end{array}$ & $\begin{array}{r}.24 \\
.13 \\
.26 \\
9.69\end{array}$ & $\begin{array}{r}.13 \\
.01 \\
.53 \\
11.06\end{array}$ & $\begin{array}{r}.12 \\
.01 \\
.57 \\
11.89\end{array}$ & & 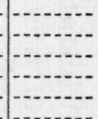 \\
\hline $\mathrm{CH}_{3} \mathrm{COO}$ & & & & & & & 17.88 & 19.09 & & & & & & & & & & \\
\hline $\begin{array}{r}\text { Basicity (oH-) } \\
\text { (equiv/liter) }\end{array}$ & 0.246 & 0.270 & 0.123 & 0.119 & 0.051 & 0.042 & 0.068 & 0.062 & $\begin{array}{l}0.03 \\
.222\end{array}$ & $\begin{array}{l}0.02 \\
.270\end{array}$ & 0.267 & 0.283 & 0.149 & 0.220 & 0.255 & 0.273 & & \\
\hline
\end{tabular}


Only a few random analyses were made on filtrates from duplicate pastes. These may be taken as indicative of the reproducibility of the data. The analyses of extracts from duplicate pastes obtained at 7 minutes (clinker 11 plus calcium acetate) were as follows:
$\begin{array}{rr}\mathrm{SiO}_{2} & \text { g/liter } \\ \mathrm{D} \mathrm{O}_{2} & 001 \\ \mathrm{R}_{2} \mathrm{O}_{3} \ldots \ldots+ & 001\end{array}$
Do
$\mathrm{CaO}$
$\mathrm{SO}_{3} \mathrm{Do}$
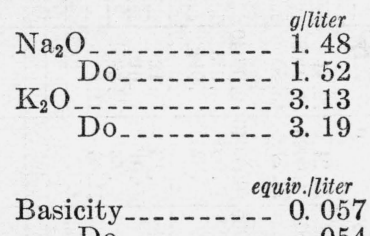

In general, these data show good agreement. The observed differences are due probably more to the variations of about 1 or 2 minutes in the times of contact of solution and clinker than to analytical errors. A few determinations of $\mathrm{CaO}$ and basicity in filtrates from duplicate pastes at 2 hours showed better agreement than those observed at 7 minutes.

The differences between the weights of the water used in making up the pastes and the weights of the filtrates given in table 3 furnish no indication of the extent of hydration of the clinkers, since most of the uncombined water could not be removed by the method used. This was particularly true of some of the 7 -minute pastes, where shrinkage caused by quick setting interfered with the filtration process.

The filtrates were clear and colorless with the following exceptions: (1) A yellow color, observed in some of the extracts, was found to be associated with the presence of $\mathrm{Cr}_{2} \mathrm{O}_{3}$. (2) Triethanolamine, after reaction with the clinkers, usually imparted a light-blue color. (3) The use of calcium acetate with a number of clinkers resulted in the formation of a white colloidal solution.

$\mathrm{MgO}$ was generally absent from the solution $(0.000 \mathrm{~g} /$ liter in 173 out of 204 determinations) or present only in small amounts $(0.001$ to $0.029 \mathrm{~g} /$ /iter). Since no correlations involving $\mathrm{MgO}$ could be deduced, it will not be considered further.

Sulfite was present in solutions obtained from the pastes of clinkers 7 and 8 (no added materials). Small amounts of thiosulfate were present in the filtrates from corresponding pastes containing gypsum. No quantitative estimation of these two constituents was attempted.

\section{COMPOSITIONS OF THE FILTRATES}

Figure 1, plotted from data in table 3 , shows the compositions of the 7 -minute and 2-hour extracts from the clinker pastes containing: no added materials. In this figure the basicities of the extracts and concentrations of each oxide are plotted against the potash content of the clinkers from which the extracts were obtained.

Although this method of plotting the data is somewhat empirical, it appears to be justified in that it reveals certain trends in the data. Thus the increase in ccncentration of potash in solution with increased potash content of the clinkers is accompanied by increased basicity; increased concentrations of silica and oxides of the $\mathrm{R}_{2} \mathrm{O}_{3}$ group; increased concentration of sultate in the extracts from the clinkers highest in potash; and a rather irregular decrease in the lime con- 
centration. The graph of soda concentrations serves only for comparison of the individual values with the concentrations of other oxides, since the quantities of extracted soda have no relation to the potash content of the clinkers.

The percentage extractions of soda from the clinkers are considerably smaller than the extractions of potash and show less relation to the soda content of the clinkers. Hence the adopted method of plotting gives more regular relations than those which result when the concentrations of the various oxides in the extracts are plotted against the total alkali content of the clinkers.

In this section the discussion will be confined to the salient trends in the concentrations of the individual oxides in the extracts from the pastes both with and without added materials. The general effects of each added material on the concentrations of all of the oxides taken as a group will be discussed in section 4, page 240 .

\section{(a) ALKALIES $\left(\mathrm{K}_{2} \mathrm{O}\right.$ AND $\left.\mathrm{Na}_{2} \mathrm{O}\right)$}

As shown by figure 1, the maximum concentrations of alkalies in the extracts from the untreated clinker pastes were $2.99 \mathrm{~g} /$ liter of $\mathrm{Na}_{2} \mathrm{O}$ and $18.65 \mathrm{~g} /$ liter of $\mathrm{K}_{2} \mathrm{O}$ at 7 minutes and $3.89 \mathrm{~g} /$ /iter of $\mathrm{Na}_{2} \mathrm{O}$ and $19.71 \mathrm{~g} /$ liter of $\mathrm{K}_{2} \mathrm{O}$ at 2 hours. The relatively large amounts of $\mathrm{K}_{2} \mathrm{O}$ in the extracts from clinkers $4,5,6,11$, and 12 are attributed to the presence of $\mathrm{K}_{2} \mathrm{SO}_{4}{ }^{4}$ In general, smaller amounts of alkalies were extracted in the interval between 7 minutes and 2 hours than during the first 7 minutes.

The results indicate that large differences may exist among clinkers with respect to the rate of solution of the alkalies. In all the 12 clinkers considerable amounts of alkalies remained undissolved at 2 hours. The extent of solution is best shown by calculations of the fractions (percentages of the total in the clinkers) of potash and soda that had dissolved at 7 minutes and at 2 hours.

Such calculations were made for the filtrates from the untreated pastes and from those containing gypsum and triethanolamine. These two added materials represent the extreme effects of the addition agents on the extractions of alkalies - gypsum causing the greatest decreases and triethanolamine the greatest increases. The results given in table 4 shows that the percentages of soda and potash in solution at 7 minutes varied from 0.4 to 30 and 1 to 58 , respectively. Calculations based on the data in table 4 reveal that the rate of solution of $\mathrm{K}_{2} \mathrm{O}$ from clinkers between 7 minutes and 2 hours varied between 0 and 9 percent, and of $\mathrm{Na}_{2} \mathrm{O}$, between 1 and 12 . The values with added gypsum were slightly decreased, from 1 to 8 percent for the $\mathrm{K}_{2} \mathrm{O}$ and 1 to 7 percent for the $\mathrm{Na}_{2} \mathrm{O}$. In the presence of triethanolamine, the rate of solution of the $\mathrm{K}_{2} \mathrm{O}$ (from six clinkers) varied between 2 and 10 percent, and of $\mathrm{Na}_{2} \mathrm{O}$, from 2 to 21 percent.

\footnotetext{
4 The assumption that all, or nearly all, the $\mathrm{SO}_{3}$ is present as $\mathrm{K}_{2} \mathrm{SO}_{4}$ in clinkers 4, 5, 6, 11, and 12 is based on the following deductions, as previously reported in detail [1]. The large fractions (about 50 percent) of total $\mathrm{K}_{2} \mathrm{O}$ dissolving in 7 minutes, together with a nearly linear relation between the $\mathrm{SO}_{3}$ and $\mathrm{K}_{2} \mathrm{O}$ contents of the clinkers, suggested the presence of the readily soluble salt, $\mathrm{K}_{2} \mathrm{SO}_{4}$. Supporting evidence indicating the presence of the salt in the clinkers was shown by the good agreement between the number of moles of $\mathrm{K}_{2} \mathrm{O}$ in the extracts and the number of moles of $\mathrm{SO}_{3}$ in the clinkers.

moles of $\mathrm{K}_{2} \mathrm{O}$ in the extracts and the number of moles of $\mathrm{SO}_{3}$ in the clinkers.
Reports by Taylor on phase-equilibria studies involving $\mathrm{K}_{2} \mathrm{O}$ and $\mathrm{SO}_{3}$ as constituents [13] and on direct examinations of commercial clinkers [14] show that $\mathrm{K}_{2} \mathrm{SO}_{4}$ generally may be observed microscopically in clinker samples if the calculated potential $\mathrm{K}_{2} \mathrm{SO}_{4}$ is more than 0.25 percent.
} 


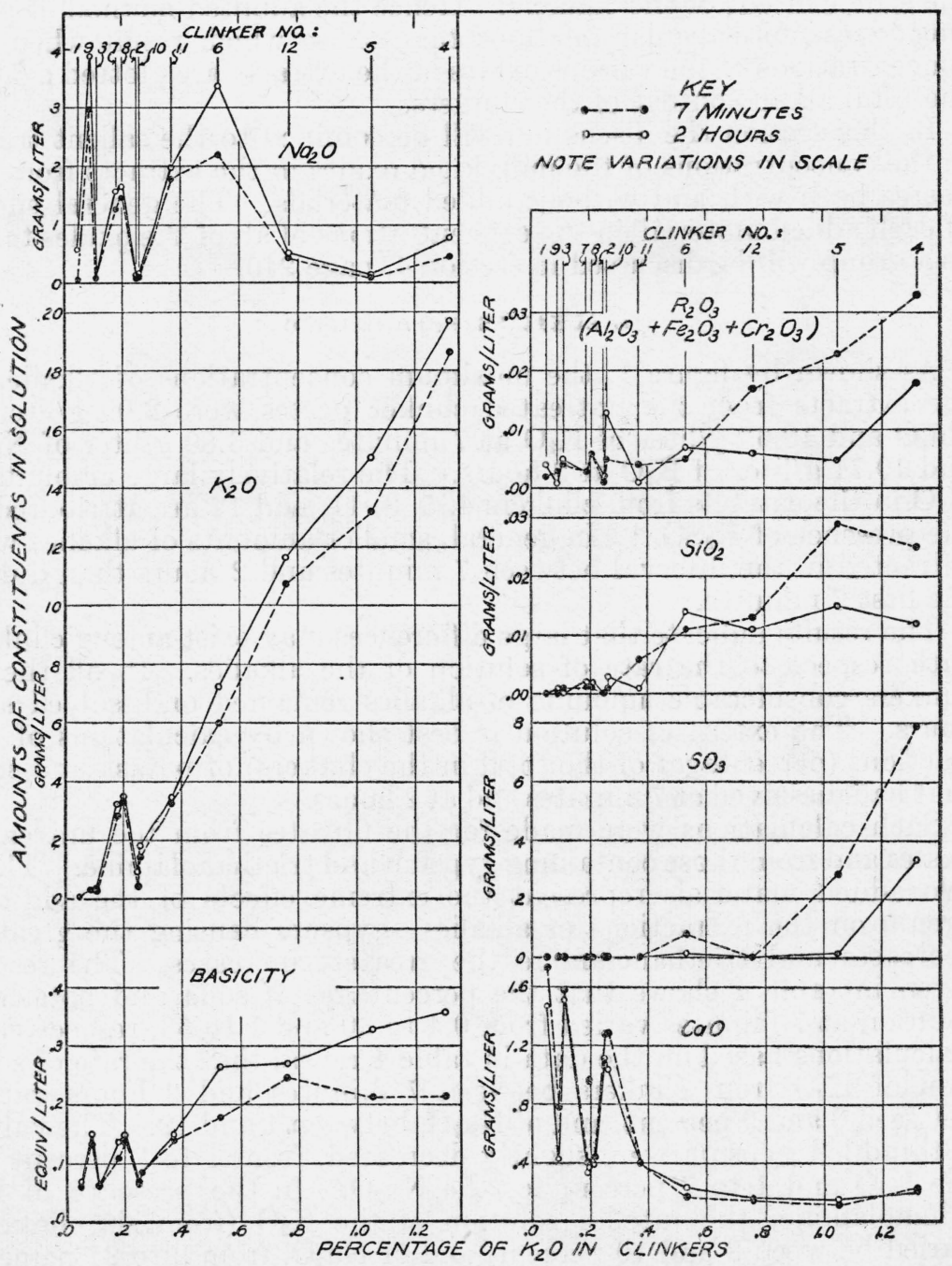

FIGURE 1.-Amounts of constituents found in filtrates from untreated clinker pastes at 7 minutes and 2 hours

Note variations in scales 
TABLE 4.-Percentages of total $\mathrm{K}_{2} \mathrm{O}$ and total $\mathrm{Na}_{2} \mathrm{O}$ in clinkers which were found in filtrates at 7 minutes and 2 hours from pasles containing gypsum, triethanolamine, and no added materials

\begin{tabular}{|c|c|c|c|c|c|c|c|c|c|c|c|c|c|c|}
\hline \multicolumn{8}{|c|}{$\mathrm{K}_{2} \mathrm{O}$} & \multicolumn{7}{|c|}{$\mathrm{Na}_{2} \mathrm{O}$} \\
\hline \multirow{2}{*}{ Clinker } & \multirow{2}{*}{$\begin{array}{c}\text { Total } \\
\text { in } \\
\text { clinker }\end{array}$} & \multicolumn{2}{|c|}{$\begin{array}{c}\text { No Added } \\
\text { material }\end{array}$} & \multicolumn{2}{|c|}{ Gypsum } & \multicolumn{2}{|c|}{$\begin{array}{c}\text { Triethano- } \\
\text { lamine }\end{array}$} & \multirow{2}{*}{$\begin{array}{c}\text { Total } \\
\text { in } \\
\text { clinker }\end{array}$} & \multicolumn{2}{|c|}{$\begin{array}{l}\text { No Added } \\
\text { material }\end{array}$} & \multicolumn{2}{|c|}{ Gypsum } & \multicolumn{2}{|c|}{$\begin{array}{l}\text { Triethano- } \\
\text { lamine }\end{array}$} \\
\hline & & $7 \mathrm{~min}$ & $2 \mathrm{hr}$ & $7 \mathrm{~min}$ & $2 \mathrm{hr}$ & $7 \mathrm{~min}$ & $2 \mathrm{hr}$ & & $7 \mathrm{~min}$ & $2 \mathrm{hr}$ & $7 \mathrm{~min}$ & $2 \mathrm{hr}$ & $7 \mathrm{~min}$ & $2 \mathrm{hr}$ \\
\hline $\begin{array}{l}0 \\
10 \\
11 \\
12\end{array}$ & $\begin{array}{c}\% \\
0.05 \\
.25 \\
.11 \\
1.32 \\
1.05 \\
0.53 \\
.18 \\
.20 \\
.09 \\
.26 \\
.37 \\
.70\end{array}$ & $\begin{array}{r}\% \\
1 \\
6 \\
9 \\
50 \\
44 \\
40 \\
56 \\
58 \\
14 \\
20 \\
31 \\
49\end{array}$ & $\begin{array}{r}\% \\
6 \\
14 \\
10 \\
52 \\
50 \\
49 \\
62 \\
61 \\
14 \\
25 \\
33 \\
54\end{array}$ & $\begin{array}{r}\%_{1} \\
4 \\
5 \\
48 \\
43 \\
35 \\
43 \\
54 \\
11 \\
19 \\
23 \\
41\end{array}$ & $\begin{array}{r}\%_{5} \\
7 \\
8 \\
51 \\
46 \\
41 \\
51 \\
59 \\
13 \\
22 \\
25 \\
42\end{array}$ & \begin{tabular}{|r|}
$\%_{1}$ \\
8 \\
13 \\
\hdashline \\
\hdashline 15 \\
22 \\
37 \\
\end{tabular} & $\begin{array}{r}\% \\
3 \\
14 \\
22 \\
68 \\
59 \\
59 \\
87 \\
23 \\
32 \\
40 \\
\end{array}$ & $\begin{array}{c}\% \\
0.56 \\
.04 \\
.02 \\
.09 \\
.05 \\
.62 \\
.18 \\
.17 \\
.87 \\
.13 \\
.43 \\
.12\end{array}$ & \begin{tabular}{|c|}
$\%$ \\
0.4 \\
12 \\
10 \\
19 \\
8 \\
12 \\
25 \\
30 \\
12 \\
7 \\
15 \\
13
\end{tabular} & $\begin{array}{c}\% \\
3.8 \\
18 \\
12 \\
31 \\
12 \\
19 \\
31 \\
34 \\
16 \\
9 \\
16 \\
15\end{array}$ & \begin{tabular}{|c|}
$\%$ \\
0.4 \\
8 \\
8 \\
13 \\
7 \\
12 \\
17 \\
22 \\
10 \\
5 \\
6 \\
8
\end{tabular} & $\begin{array}{c}\% \\
1.7 \\
11 \\
9 \\
20 \\
11 \\
16 \\
19 \\
26 \\
13 \\
6 \\
8 \\
10\end{array}$ & \begin{tabular}{|c|}
$\%$ \\
1.3 \\
10 \\
16 \\
\hdashline \\
\\
\\
14 \\
7 \\
23
\end{tabular} & $\begin{array}{r}\% \\
18 \\
18 \\
31 \\
96 \\
33 \\
32 \\
52 \\
35 \\
16 \\
25 \\
.\end{array}$ \\
\hline
\end{tabular}

The results in table 4 were computed on the assumption that the ratio of clinker to solution remained unchanged up to 2 hours. Actually, a portion of the water had combined with the clinker during this period, having the effect of increasing the concentrations of the solutes. The extent of this hydration at time periods comparable to those used in this investigation has been studied by Forsen [15]. He reported that 5 and 6 percent of the mixing water combined as firmly fixed water (retained at $105^{\circ} \mathrm{C}$ ) in 10 minutes and 1 hour, respectively, in the case of a quick-setting clinker gaged with 40 percent of water. Addition of a retarder (gypsum plus plaster of paris) reduced these values to 4 and 5 percent, respectively. These results are mentioned here only to indicate roughly the fraction of "fixed" water and not as correction factors. It is apparent that the amount of water removed as hydrate water will vary from clinker to clinker and with the added materials. Since no corrections were made for hydrate water, the computations are intended only as approximations of the true values.

\section{(b) LIME}

The relation of lime concentration to basicity indicated by the comparisons in figure 1 is more clearly shown in figure 2 , where the amounts of lime are plotted against the values of basicity $(\mathrm{NaOH}$ $\left.+\mathrm{KOH}+\mathrm{Ca}(\mathrm{OH})_{2}\right)$ of the extracts from untreated pastes. The equilibrium solubility curves of lime in sodium and potassium hydroxide solutions, determined by Forsen [15], are reproduced in this figure.

The trend of the data of this study roughly parallels Forsen's solubility curves. The amounts of $\mathrm{CaO}$ in solution, however, exceeded the normal saturation values in ten of the twelve 7-minute extracts and in all of the 2-hour extracts. The data do not fall on a smooth curve because the degree of supersaturation undoubtedly varied among the filtrates. Also in the case of the high-potash clinkers the presence of $\mathrm{SO}_{3}$ may have affected the solubility relations.

A qualitative verification of the supersaturation of the solutions was obtained by visually estimating the amounts of crystalline ma- 


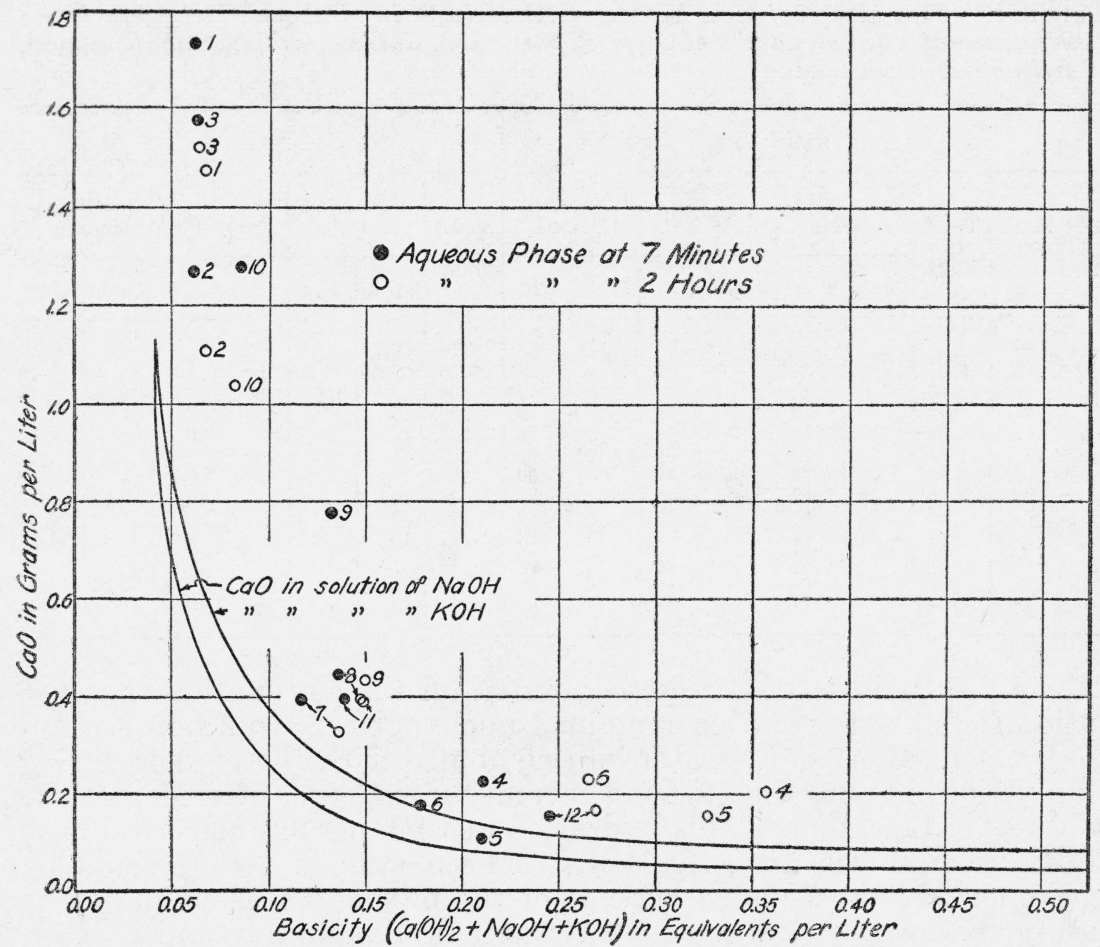

Figure 2.-Plot of CaO-basicity data for filtrates from untreated clinker pastes compared with solubilities of $\mathrm{Ca}(\mathrm{OH})_{2}$ in pure solutions of $\mathrm{NaOH}$ and $\mathrm{KOH}$ reported by Forsen [13].

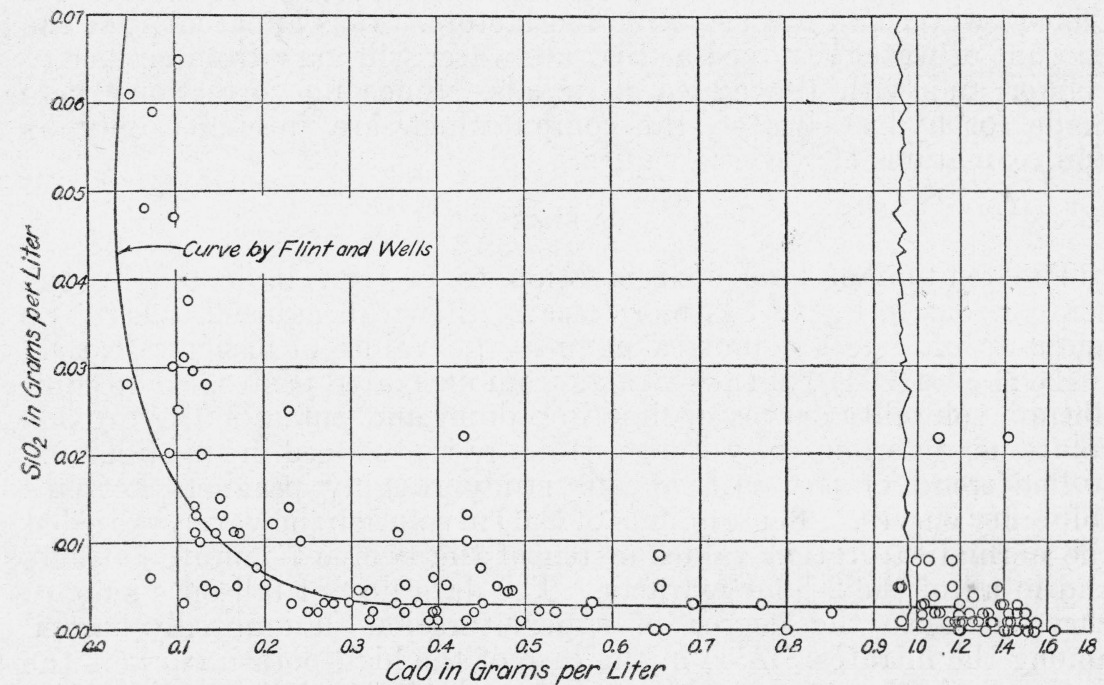

FIgURe 3.-Plot of $\mathrm{CaO}-\mathrm{SiO}_{2}$ data for filtrates from clinker pastes with and without added materials compared with the $\mathrm{CaO}-\mathrm{SiO}_{2}$ correlation reported for the system $\mathrm{CaO}-\mathrm{SiO}_{2}-\mathrm{H}_{2} \mathrm{O}$ by Flint and Wells [14]. 
terial precipitated after 2 months from the various filtrates sealed in glass tubes. The crystals, identified as $\mathrm{Ca}(\mathrm{OH})_{2}$, were as much as $1 \mathrm{~mm}$ in cross section.

\section{(c) SILICA}

Figure 1 shows that an increased concentration of silica in the extracts was accompanied by a decreased concentration of lime. The relation is more evident in figure 3 , where the concentration of silica is plotted against that of lime in all of the extracts except those from pastes containing calcium chloride, calcium acetate, and fluosilicic acid. The correlation of the data with the curve given by Flint and Wells [16] for the solubility of silicia in lime solutions at $30^{\circ} \mathrm{C}$ is noteworthy. Apparently the effect of the alkalies on the lime concentration, illustrated in figure 2 , does not greatly alter the silica-lime relation. The spread in the points is not surprising, considering the complex nature of the solutions, the lack of attainment of equilibrium, and relatively large analytical errors in the determination of the small amounts of $\mathrm{SiO}_{2}$.

Flint and Wells [16] and Bessey [17] have demonstrated that the compositions of the hydrous calcium silicates are dependent on the composition of the solution. Thus, as the concentration of lime in solution is decreased, the precipitated calcium silicate hydrate becomes poorer in lime. The observed correlation of the $\mathrm{CaO}-\mathrm{SiO}_{2}$ data in the present investigation suggests that lime-poor silicates may be precipitated in the clinker pastes as the lime concentration in the solution is decreased.

\section{(d) $\mathrm{R}_{2} \mathrm{O}_{3}$ GROUP $\left(\mathrm{Al}_{2} \mathrm{O}_{3}, \mathrm{Fe}_{2} \mathrm{O}_{3}\right.$, AND $\left.\mathrm{Cr}_{2} \mathrm{O}_{3}\right)$}

Figure 1 shows that the concentration of $\mathrm{R}_{2} \mathrm{O}_{3}$ varied from 0.001 to $0.033 \mathrm{~g}$ /liter at 7 minutes and from 0.001 to $0.018 \mathrm{~g} /$ liter at 2 hours. The 7-minute filtrates showing the highest $\mathrm{R}_{2} \mathrm{O}_{3}$ contents (from clinker pastes 4,5 , and 6 ) contained $\mathrm{Cr}_{2} \mathrm{O}_{3}$; at 2 hours, however, the constituent was present only in the extract from clinker 6. All solutions containing $\mathrm{Cr}_{2} \mathrm{O}_{3}$ were yellow, indicating the presence of the chromate ion.

The portions of $\mathrm{R}_{2} \mathrm{O}_{3}$ as $\mathrm{Al}_{2} \mathrm{O}_{3}$ plus $\mathrm{Fe}_{2} \mathrm{O}_{3}$ varied from 0.001 to 0.017 $\mathrm{g} /$ liter in the filtrates from pastes containing no added materials. When the organic addition agents were incorporated in the pastes, relatively large concentrations of $\mathrm{Fe}_{2} \mathrm{O}_{3}$ and $\mathrm{Al}_{2} \mathrm{O}_{3}$ were sometimes obtained. The changes will be discussed in the section dealing with the added materials individually.

(e) $\mathrm{SO}_{3}$

As shown in figure 1 the $\mathrm{SO}_{3}$ was present in significant amounts in filtrates from clinker pastes 4,5 , and 6 at 7 minutes, but decreased in the interval between 7 minutes and 2 hours. These decreases appear to be caused by the precipitation of calcium sultoaluminate according to the reaction:

$3 \mathrm{CaO} . \mathrm{Al}_{2} \mathrm{O}_{3}{ }^{5}+3 \mathrm{~K}_{2} \mathrm{SO}_{4}+3 \mathrm{Ca}(\mathrm{OH})_{2}+31 \mathrm{H}_{2} \mathrm{O}=3 \mathrm{CaO} . \mathrm{Al}_{2} \mathrm{O}_{3} \cdot 3 \mathrm{CaSO}_{4} \cdot 31 \mathrm{H}_{2} \mathrm{O}+6 \mathrm{KOH}$.

The evidence for the occurrence of this reaction is based on calculations which show that the equivalents of $\mathrm{SO}_{4}^{-}$precipitated in the interval between 7 minutes and 2 hours are equal to the increase in

\footnotetext{
${ }_{5}^{5}$ The formula $3 \mathrm{CaO} \cdot \mathrm{Al}_{2} \mathrm{O}_{3}$ is used in this and subsequent equations for purposes of discussion only, since other aluminous materials in cement clinkers may react similarly. The trisulfate form of sulfoaluminate is considered here, but the precipitation of the monosulfate form would not alter the ionic balances.
} 
equivalents of $\mathrm{OH}^{-}$during the same interval, allowance being made for the $\mathrm{OH}^{-}$that was introduced by further changes in the concentration of the alkalies and lime. Such calculations were reported in a previous publication [1] for extracts of one of the untreated pastes. The more complete data now available are given in table 5 for the filtrates containing the larger amounts of $\mathrm{SO}_{3}$ at 7 minutes from clinker pastes alone, and with T D A, tannic acid, and fluosilicic acid as added materials. Table 5 shows that for each filtrate the total increase in $\mathrm{OH}^{-}$concentration (column 3) exceeds the total decrease in $\mathrm{SO}_{4}^{=}$ concentration (column 6). This is accounted for by the changes in the concentrations of $\mathrm{NaOH}, \mathrm{KOH}$, and $\mathrm{Ca}(\mathrm{OH})_{2}$ in the extracts between 7 minutes and 2 hours. If the $\mathrm{OH}^{-}$introduced from these sources (column 4) is deducted from the total increase in $\mathrm{OH}^{-}$concentration (column 3), the increases in $\mathrm{OH}^{-}$caused by the sulfoaluminate reaction are obtained (column 5). These values are in close agreement with the decreases in $\mathrm{SO}_{4}^{\overrightarrow{4}}$ concentration (column 6).

TABLE 5.-Changes in ionic concentrations of extracts from clinker pastes between 7 minutes and 2 hours

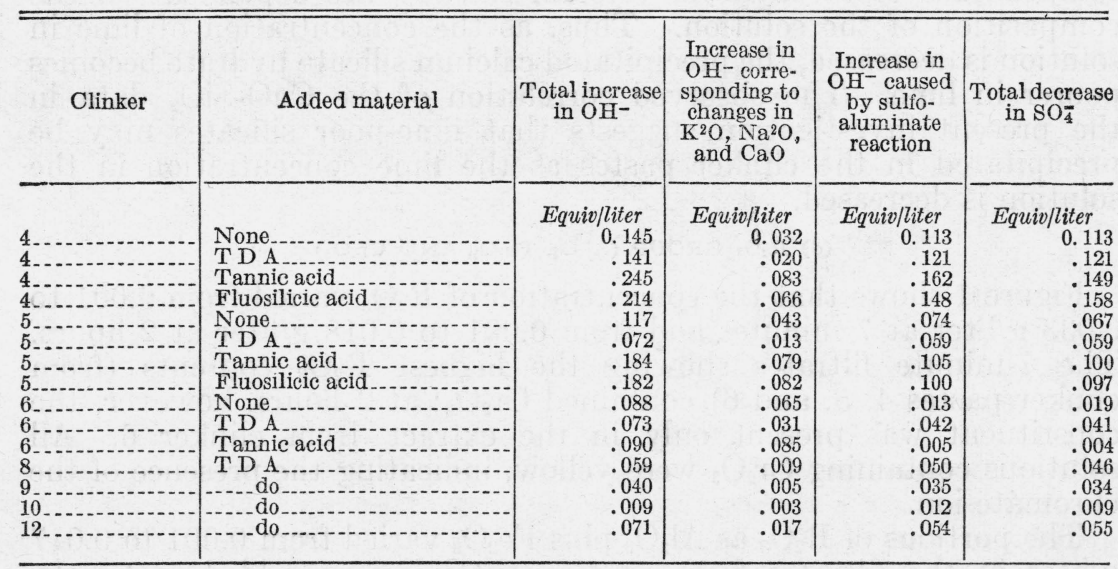

In many instances the sulfoaluminate reaction accounts for the small amounts of $\mathrm{SO}_{3}$ in the filtrates at 7 minutes. For example, the low concentration of $\mathrm{SO}_{3}(0.10 \mathrm{~g} /$ liter $)$ in the 7 -minute extract from the paste of clinker 4 containing sucrose suggests that almost complete precipitation of the $\mathrm{SO}_{3}$ as sulfoaluminate had occurred. In the extract from the corresponding paste containing no added material, the high concentration of $\mathrm{SO}_{3}(7.90 \mathrm{~g} /$ liter $)$ leads to the belief that precipitation of sulfoaluminate had proceeded more slowly.

\section{3. pH AND OTHER CONSTANTS OF THE FILTRATES}

Constant emf was not attained in many of the $\mathrm{pH}$ determinations. This may have been due to the presence of so-called "electrode poisons" (traces of sulfur compounds, ferric and chromate ions, etc.). Although as much as 6 hours was allowed frequently for approaching a constant emf, the values obtained in many determinations appeared to be low. Because of the uncertainty as to the accuracy of such results, and since the filtrates consisted essentially of mixtures of 
calcium and alkali hydroxides and their salts, the $\mathrm{pH}$ values of pure solutions of calcium hydroxide and potassium hydroxide, respectively, were determined. These values are plotted against corresponding basicities in figure 4 . The resulting curves furnish a basis for comparison with the data on filtrates from the clinker pastes, with and without added materials, plotted in the same figure.

The two curves extending from $\mathrm{pH} 11.95$ to 12.53 and from 12.55 to 13.60 are those for pure solutions of calcium hydroxide and potassium hydroxide, respectively. These curves do not meet because potassium hydroxide is the stronger of the two bases and, at an equivalent concentration, has the higher $\mathrm{pH}$. Thus at a basicity of 0.04 equiv/liter, corresponding to that of a saturated lime solution, the curves show a difference in $\mathrm{pH}$ of 0.04 unit. However, above about 0.15 equiv/liter, the $\mathrm{pH}$ values of solutions containing both hydroxides are practically the same as those shown by the upper curve because of the low solubility of calcium hydroxide in potassium hydroxide solutions. The $\mathrm{pH}$ values of the filtrates from tbe clinker pastes, therefore, show greater deviations from the potassium hydroxide curve at the lower basicities than at the higher.

Figure 4 includes the $\mathrm{pH}$ values of practically all filtrates except those containing triethanolamine. The use of this addition agent resulted in such large potential disturbances that emf measurements could not be made. The rather consistently low values obtained on filtrates when TDA was present are also attributed to electrode poisons. Practically all the other low results on the filtrates, except those containing the calcium salts as added materials, may result from the same cause.

The $\mathrm{pH}$ values, in the absence of electrode poisons, are approximately represented by the potassium hydroxide curve in figure 4, allowance being made at the lower basicities for the presence of calcium hydroxide in the solutions.

The effect of highly soluble calcium salts in lowering the $\mathrm{pH}$ of the extracts by relatively large amounts is illustrated in figure 4, where the $\mathrm{pH}$ values of filtrates containing calcium chloride and calcium acetate are seen to lie below the $\mathrm{pH}$ curve. These decreases in $\mathrm{pH}$ are caused by the decreased effective concentration of the hydroxyl ion in the solutions.

Gypsum, because of its lower solubility, decreased the $\mathrm{pH}$ by only 0.02 to 0.04 unit at the basicities prevailing in the extracts.

The excellent agreement between the $\mathrm{pH}$ values for $\mathrm{Ca}(\mathrm{OH})_{2}$ solutions, from 0.04 to 0.015 equiv/liter, reported by Lea and Bessey [18] and those reported here is of interest because different methods of calculating $\mathrm{pH}$ were employed.

In figure 5 the specific conductivity is plotted against basicity of the filtrates from a representative number of clinker pastes with and without added materials. The relation is approximately linear for most of the extracts consisting essentially of solutions of potassium, sodium, and calcium hydroxides, such as many of the filtrates from the pastes alone and from all of those containing sucrose and triethanolamine. The introduction of relatively large amounts of sulfates, chlorides, or acetates in some of the extracts, resulting from the addition of calcium salts, accounts for the increased conductivity of such solutions.

$508748-43-6$ 


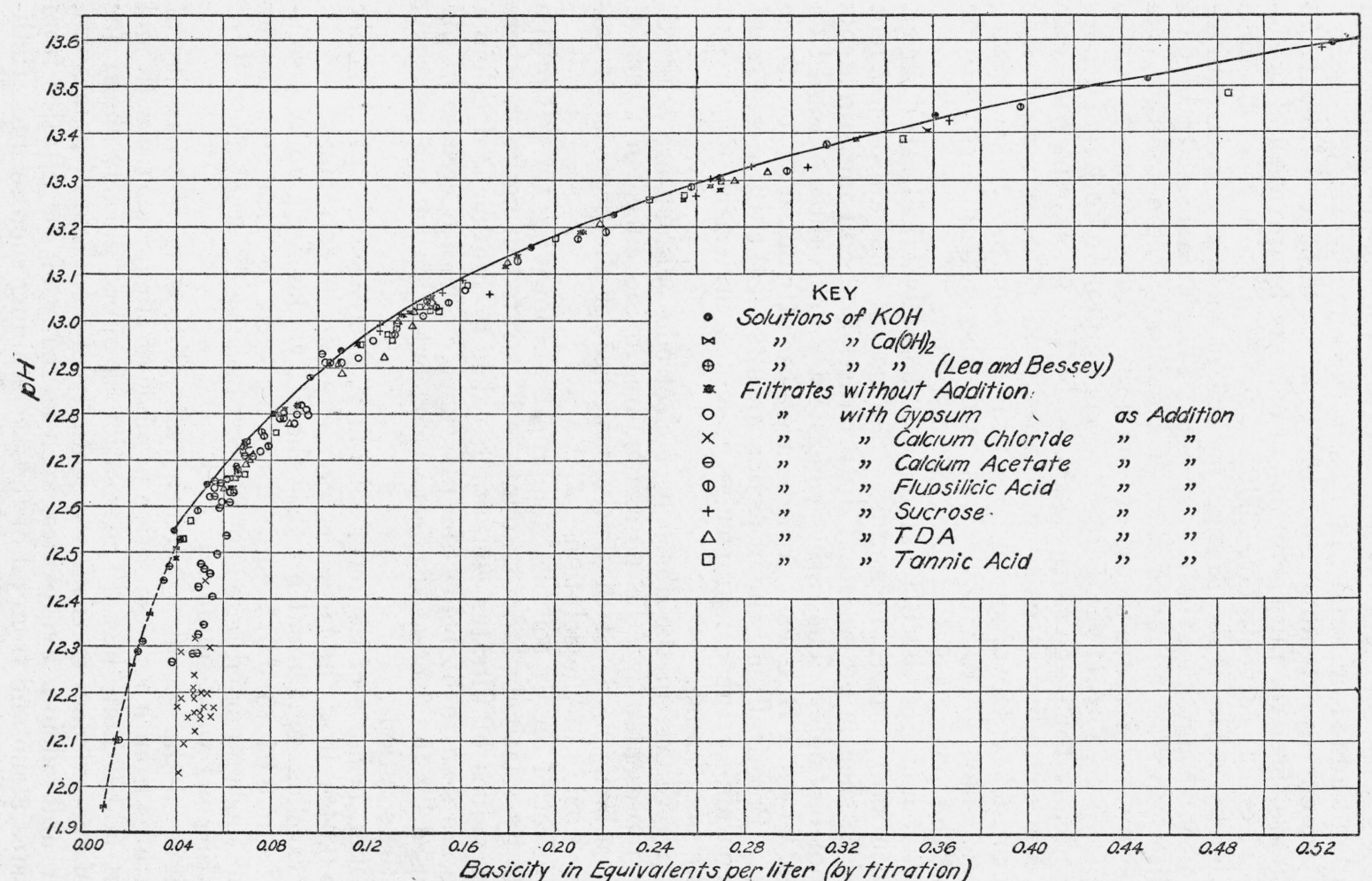

FIGURE 4.-Plot of pH-basicity data for filtrates from clinker pastes with and without added materials compared with the $\mathrm{pH}$ of pure solutions of $\mathrm{Ca}(\mathrm{OH})_{2}$ and $\mathrm{KOH}$. 


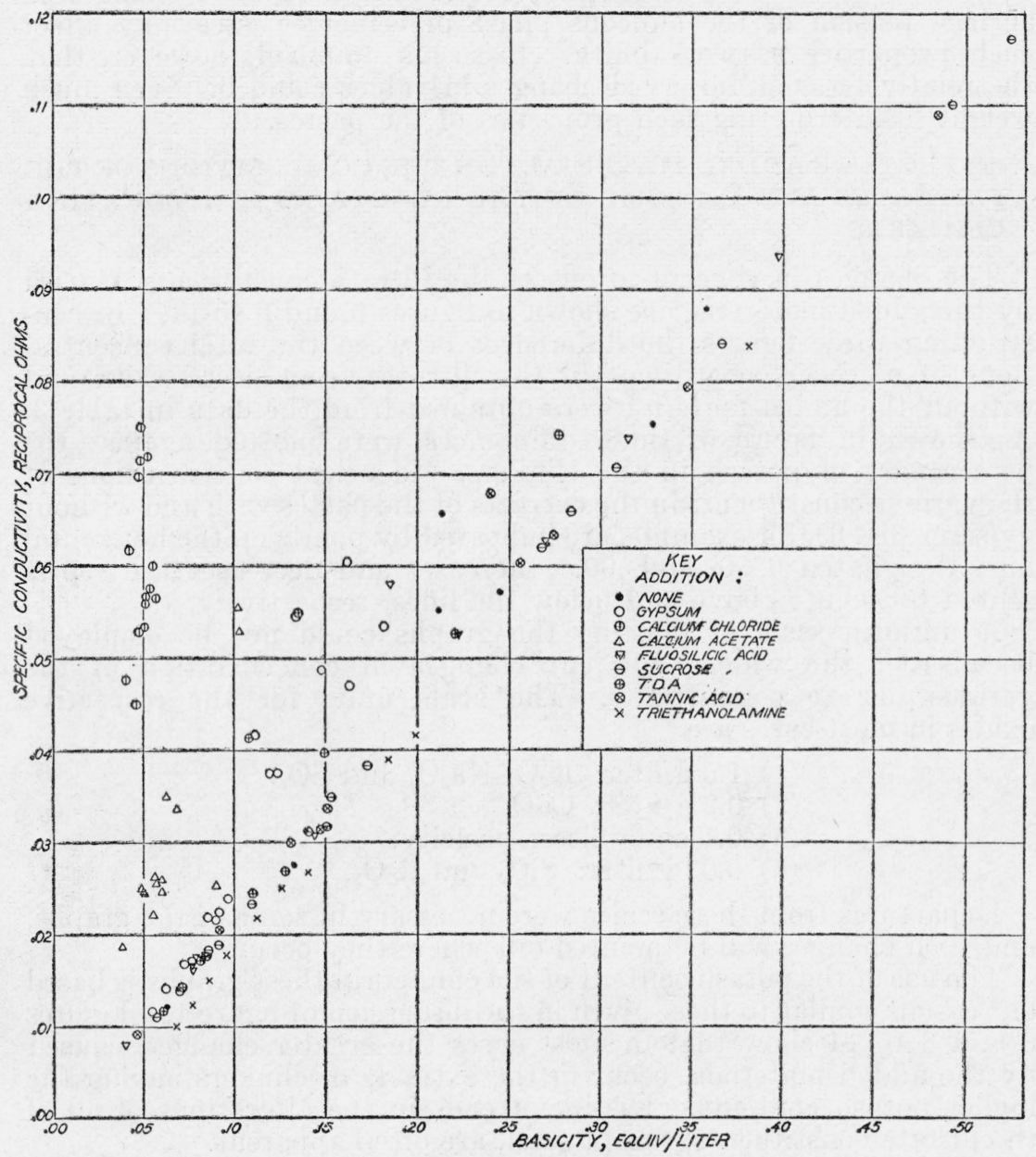

FIGURE 5.-Conductivity and basicity of filtrates from clinker pastes with and without added materials. 
The densities of the extracts, required for calculating the surface tensions, are included in table 3 , in order that the analytical data, if desired, may be expressed on a weight basis.

The surface-tension data, neglecting those below 72.0 dynes $/ \mathrm{cm}$, which were known to be low because of the presence of surface contaminants, range from 72.0 to 73.0 dynes $/ \mathrm{cm}$. The higher values were obtained on solutions containing calcium chloride and acetate as additions. It has occasionally been suggested that changes in surface tension of the aqueous phase of cement pastes may affect such properties as workability. It seems doubtful, however, that the relatively small observed changes in values could be given much weight in interpreting such properties of the pastes.

\section{EFFECTS OF ADDED MATERIALS ON THE COMPOSITIONS OF THE FILTRATES AND ON SOME OF THE REACTIONS IN HYDRATING CLINKERS}

The changes in concentrations of the filtrate constituents, caused by the added materials, are shown in figures 6 and 8 to 14 . In constructing these figures the differences between the total concentrations of a given constituent in the filtrates from pastes with and without the added material were obtained from the data in table 3. As shown in figure 6, these differences were plotted against the percentages of potash in the clinkers. Identical concentrations of the various constituents in the extracts of the pastes with and without gypsum (fig. 6), for example, are indicated by points on the horizontal lines designated $0, .0$, and .00 . Increases and decreases are represented by points above and below the lines, respectively.

A uniform scale of plotting the graphs could not be employed because of the widely different changes in concentration of the various filtrate constituents. The scale units for the respective oxides in most cases are

(1) $1.0 \mathrm{~g} /$ liter: $\mathrm{K}_{2} \mathrm{O}, \mathrm{Na}_{2} \mathrm{O}$, and $\mathrm{SO}_{3}$.

(2) $0.2 \mathrm{~g} /$ liter: $\mathrm{CaO}$.

(3) 0.1 equiv/liter: basicity.

(4) $0.02 \mathrm{~g} /$ liter: $\mathrm{SiO}_{2}$ and $\mathrm{R}_{2} \mathrm{O}_{3}$.

Departures from these scales were necessary in some of the graphs, and such changes will be pointed out where they occur.

The use of the potash content of the clinkers in these graphs is based on reasons similar to those given in the discussion of figure 1. Figures 6 and 8 to 14 show that in most cases the greater changes, caused by the added materials, occur in the extracts of clinkers having the higher potash contents. Distinct trends in the alterations of all of the filtrate constituents, except soda, are often apparent.

It should be pointed out that small differences between the compositions of filtrates from pastes with and without added materials may not necessarily indicate effects of the additions but may be caused by difficulties in duplicating the manner of mixing the pastes or the time of filtration. Although the time of filtration of duplicate pastes did not vary more than 1 or 2 minutes, as already mentioned, greater variations were experienced in that of pastes made from clinkers with and without added materials. Some of the pastes which became quick-setting in the presence of added materials, had to be mixed more vigorously and often required 2 to 5 minutes longer for filtration than companion pastes without additions. 


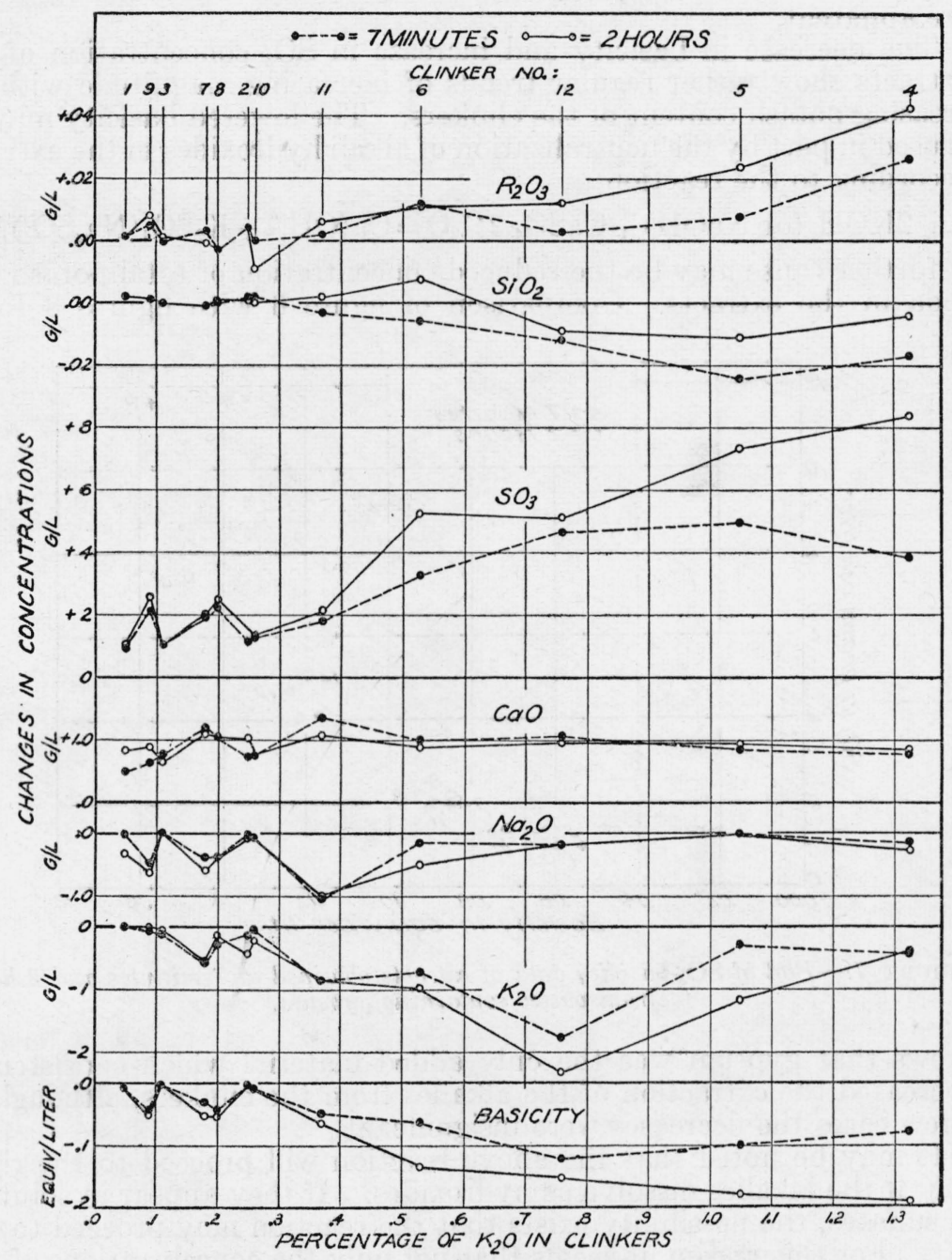

Figure 6.-Changes in compositions of extracts caused by gypsum. Note variations in scale: 


\section{(a) GYPSUM}

The changes in concentration of the filtrate constituents, shown in figure 6, represent differences in composition of the extracts from the cements and the corresponding clinkers. Trends of decreased basicity, lowered concentrations of alkalies and silica, and increased concentrations of lime, sulfate, and $\mathrm{R}_{2} \mathrm{O}_{3}$ in the extracts of the cements are apparent.

The decrease in basicity and increase in $\mathrm{SO}_{3}$ concentration of the extracts show rather regular trends of increasing magnitude with increasing potash content of the clinkers. The lowered basicity may be caused in part by the neutralization of alkali hydroxides in the extracts according to the reaction:

$$
2 \mathrm{KOH} \text { (or } \mathrm{NaOH})+\mathrm{CaSO}_{4} \cdot 2 \mathrm{H}_{2} \mathrm{O}=\mathrm{Ca}(\mathrm{OH})_{2}+\mathrm{K}_{2} \mathrm{SO}_{4}\left(\mathrm{Na}_{2} \mathrm{SO}_{4}\right) \text {. }
$$

A further cause may be the reduced concentration of total potash and soda in the extracts. Comparison of figure 6 with figures 8 to 14

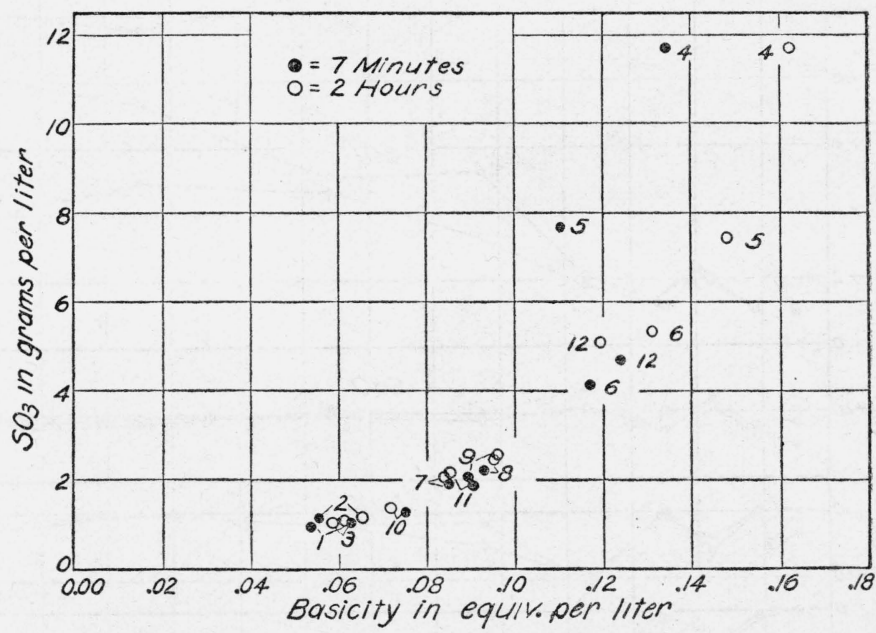

Figure 7.-Plot of $\mathrm{SO}_{3}$-basicity data of extracts obtained at 7 minutes and 2 hours from pastes containing gypsum.

shows that gypsum was the only added material which consistently decreased the extraction of the alkalies from the clinkers, although in a few cases the decreases were insignificant.

It may be noted that the above reaction will proceed to the right only if the alkalies dissolve as hydroxides. If they appear in solution as sulfates, the possibility exists that the reaction may proceed to the left. For this reason, it seems that not only the concentrations of the alkali hydroxides but also the possible concentrations of sulfate merit consideration in making interpretations based on the reaction. The relation between basicity and sulfate concentration of the extracts is shown by a plot of the basicity- $\mathrm{SO}_{3}$ data in figure 7 . This figure shows that relatively high basicities may prevail in the extracts even when gypsum is present in the pastes.

Decreases in the concentration of silica and increases in that of $\mathrm{R}_{2} \mathrm{O}_{3}$ group are indicated in figure 6 . As shown by analysis, the increases in the concentrations of $\mathrm{R}_{2} \mathrm{O}_{3}$ in the extracts from clinkers 
$4,5,6,9$, and 12 were due to the presence of $\mathrm{Cr}_{2} \mathrm{O}_{3}$. The yellow color of the other filtrates suggests that they also contained $\mathrm{Cr}_{2} \mathrm{O}_{3}$ but in amounts of less than $0.005 \mathrm{~g} /$ liter, this being the lowest concentration detectable by the procedure used.

\section{(b) CALCIUM CHLORIDE}

Comparison of the decreases in basicity in the extracts in figures 8 and 6 indicates that calcium chloride produced larger changes than did gypsum. This may be due to the greater solubility of calcium chloride, which permitted more complete neutralization of the alkali hydroxides, according to the equation:

$$
2 \mathrm{KOH} \text { (or } \mathrm{NaOH})+\mathrm{CaCl}_{2}=\mathrm{Ca}(\mathrm{OH})_{2}+2 \mathrm{KCl} \text { (or } \mathrm{NaCl} \text { ). }
$$

Calcium chloride also differed from gypsum in that, on the average, it increased the extractions of the alkalies from the clinkers. At 2 hours, particularly, the trend of increased potash concentration is very uniform.

In the presence of calcium chloride, the sulfoaluminate reaction appears to be greatly accelerated, as indicated by the large decreases in $\mathrm{SO}_{3}$ concentration, leaving only small amounts in solution. The sulfoaluminate may precipitate from pastes of clinkers containing potassium sulfate, according to the reaction:

$$
3 \mathrm{CaCl}_{2}+3 \mathrm{~K}_{2} \mathrm{SO}_{4}+3 \mathrm{CaO} . \mathrm{Al}_{2} \mathrm{O}_{3}+31 \mathrm{H}_{2} \mathrm{O}=3 \mathrm{CaO}_{2} . \mathrm{Al}_{2} \mathrm{O}_{3} \cdot 3 \mathrm{CaSO}_{4} \cdot 31 \mathrm{H}_{2} \mathrm{O}+6 \mathrm{KCl} \text {. }
$$

This reaction would account for the smaller amounts of lime present in the extracts from the clinkers containing potassium sulfate.

The quantities of lime remaining in solution are governed by the extent of precipitation of calcium chloroaluminate as well as by the neutralization and sulfoaluminate reactions. The formation of the chloroaluminate proceeds according to the equation:

$$
3 \mathrm{CaO} \cdot \mathrm{Al}_{2} \mathrm{O}_{3}+\mathrm{CaCl}_{2}+10 \mathrm{H}_{2} \mathrm{O}=3 \mathrm{CaO} \cdot \mathrm{Al}_{2} \mathrm{O}_{3} \cdot \mathrm{CaCl}_{2} \cdot 10 \mathrm{H}_{2} \mathrm{O} \text {. }
$$

General trends of lowered concentrations of both silica and $\mathrm{R}_{2} \mathrm{O}_{3}$ occurred in the extracts containing calcium chloride. In most cases the decreases were greater at 7 minutes than at 2 hours.

The decreases in the concentration of the chlorine from that added as calcium chloride (26.62 $\mathrm{g}$ of $\mathrm{Cl} /$ liter) are attributed largely to the formation of the chloroaluminate. Previous work [7] has shown that the composition of this compound does not vary much from the formula $3 \mathrm{CaO} \cdot \mathrm{Al}_{2} \mathrm{O}_{3} \cdot \mathrm{CaCl}_{2} 10 \mathrm{H}_{2} \mathrm{O}$ in the presence of concentrations of calcium chloride such as prevail in the extracts. Assuming that the chlorine is precipitated only as the chloroaluminate,

\begin{tabular}{|c|c|c|c|c|c|c|c|c|c|c|c|c|}
\hline Clinker..- & 1 & 2 & 3 & 4 & 5 & 6 & 7 & 8 & 9 & 10 & 11 & 12 \\
\hline 7 minutes & 3. 0 & 3. 2 & 4.8 & 2. 6 & 2. 7 & 4. 2 & 4. 7 & 5.3 & 7. 3 & 7. 3 & 4. 7 & 5. 3 \\
\hline 2 hours & 10.1 & 8.7 & 9.5 & 4.3 & 4.2 & 6.5 & 8.9 & 7.3 & 10.4 & 9.6 & 8.6 & 7.2 \\
\hline
\end{tabular}
calculations show that the percentages of the total $\mathrm{Al}_{2} \mathrm{O}_{3}$ which had reacted were as follows:

These results indicate that only small amounts of $\mathrm{Al}_{2} \mathrm{O}_{3}$ reacted with the calcium chloride during the two time periods. 


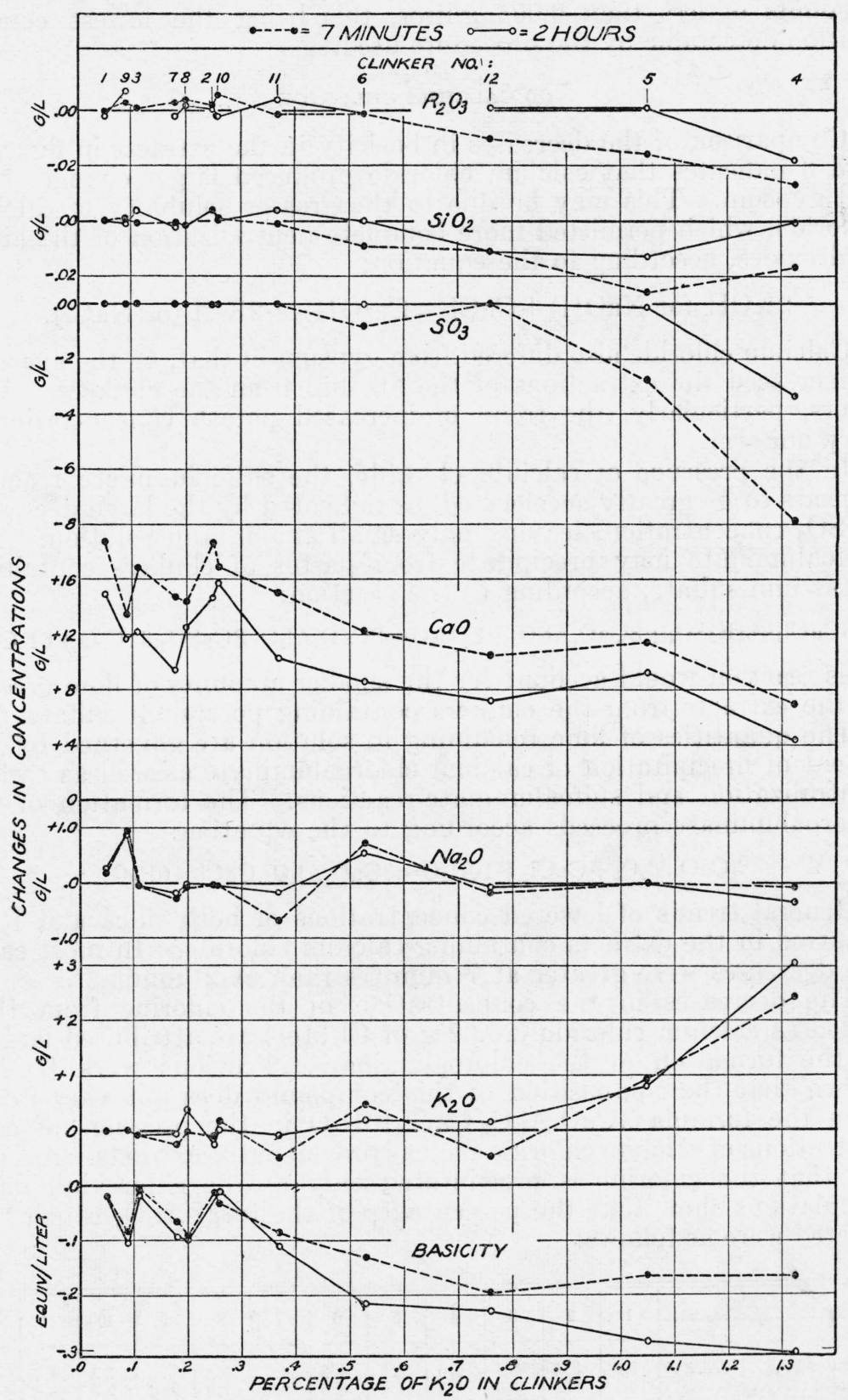

FIGURE 8.-Changes in compositions of extracts caused by calcium chloride Note variations in scale: 
(c) CALCIUM ACETATE

The changes caused by the presence of calcium acetate in the extracts are very similar both in magnitude and direction to those induced by calcium chloride as, comparison of figures 9 and 8 will reveal. As before, the basicities of many of the extracts are governed largely by the neutralization reaction between the alkali hydroxides and the added salt. The amounts of lime in the extracts (note reduced scale) are somewhat smaller than those in the extracts containing calcium chloride. It should be noted that smaller amounts of calcium acetate were added to the pastes. The remaining alterations show no marked departures from those produced by calcium chloride, except that some extracts of the low-potash clinkers contained appreciably more $\mathrm{R}_{2} \mathrm{O}_{3}$ in the presence of calcium acetate.

A white tubid appearance of ten of the twelve 7-minute extracts and of one of the 2-hour extracts indicated that some of the constituents of these solutions were present in the colloidal state.

The acetate added to the pastes was withdrawn from the extracts, probably in part by precipitation as an acetoaluminate [19] and in part by absorption, as indicated by the decreased concentration of acetate in solution at 7 minutes. Although further decreases in acetate concentration occurred between 7 minutes and 2 hours in extracts of five of the clinkers, increases were found in the six remaining extracts. No comparison is possible in the case of clinker 7 because the specified amount of calcium acetate was not added to the 2-hour paste.

\section{(d) FLUOSILICIC ACID}

The changes produced by fluosilicic acid were generally small, as shown in figure 10. Fluorine was found in the extracts in amounts varying between 0.00 to 0.11 and 0.00 to $0.18 \mathrm{~g} /$ liter at 7 minutes and 2 hours, respectively. The small amounts of fluorine in the extracts at 7 minutes show that the constituents of this material were rapidly precipitated.

The minor alterations in basicity and $\mathrm{SO}_{3}$ were decreases for the most part. Changes in the extractions of soda were small, but the extractions of potash are of interest in that they were decreased at 7 minutes and altered only slightly at 2 hours. Precipitation of calcium fluoride from the extracts may be indicated by the generally decreased concentration of lime in the extracts at both 7 minutes and 2 hours.

$A$ general trend of decreased silica and increased $\mathrm{R}_{2} \mathrm{O}_{3}$ concentration in the filtrates is apparent in figure 10.

\section{(e) SUCROSE}

Sucrose is the only one of the added materials considered thus far that increased the basicity of the extracts (fig. 11). This was caused not only by increased extraction of the alkalies but also, to a greater extent, by the almost complete conversion of dissolved potassium sulfate to potassium hydroxide, according to the sulfoaluminate reaction. Only 0.03 to $0.15 \mathrm{~g} /$ liter of $\mathrm{SO}_{3}$ remained in the 7 -minute extracts of clinkers 4,5 , and 6 , which contained the larger amounts of $\mathrm{SO}_{3}$. At the same time, the lime concentrations of 0.04 to 0.07 $\mathrm{g}$ /liter were lower than those corresponding to saturation values for the respective potassium hydroxide concentrations (see fig. 2). The 


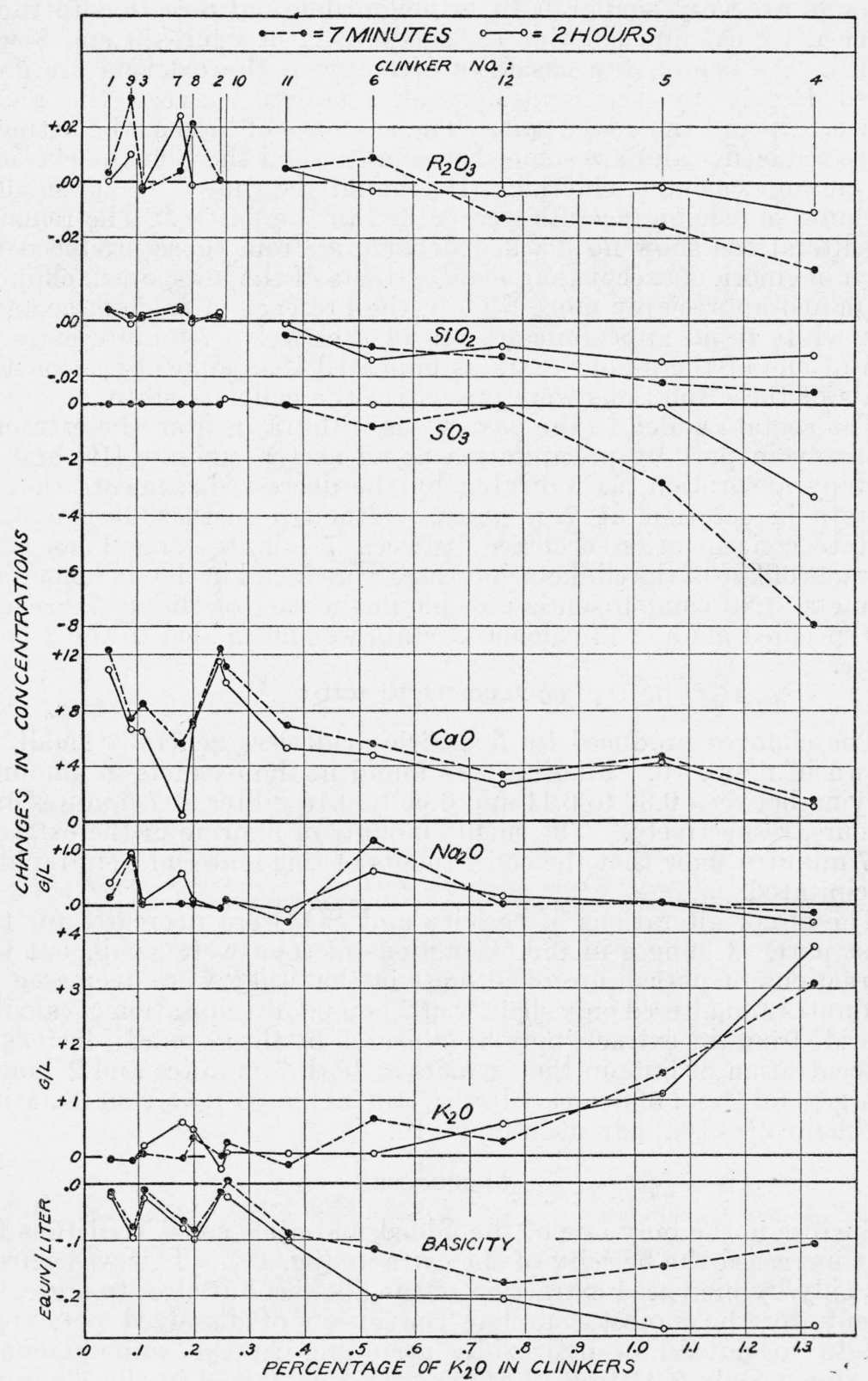

FIGURE 9.-Changes in compositions of extracts caused by calcium acetate. Note variations in scale. 


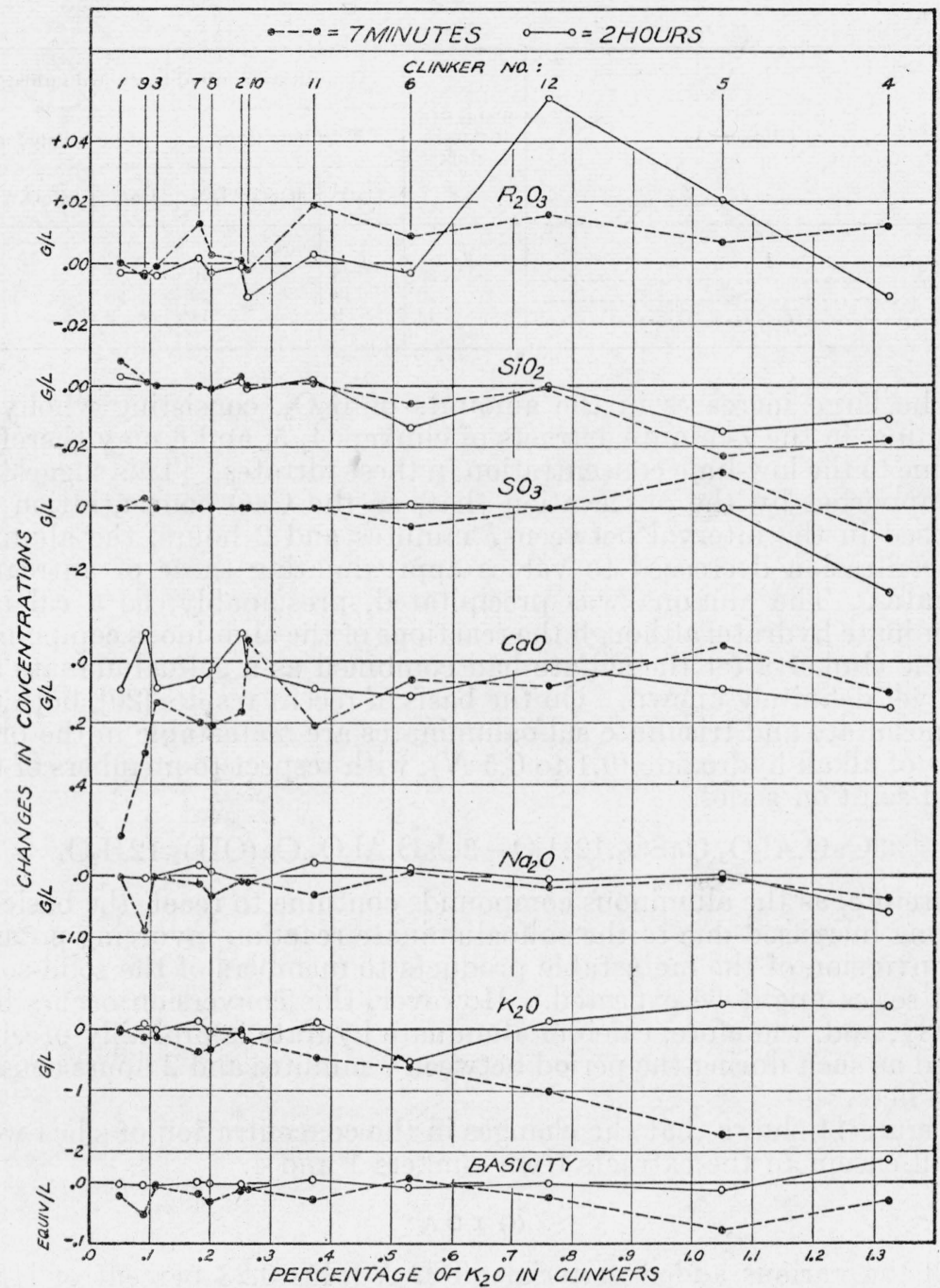

Figure 10.-Changes in compositions of extracts caused by fluosilicic acid. Note variations in scale. 
large quantities of lime required for the precipitation of the sulfoaluminates may account for the apparent undersaturation. If all the $\mathrm{SO}_{3}$ were completely extracted from each of the clinkers and precipitated either as the trisulfate form of sulfoaluminate, $3 \mathrm{CaO} \cdot \mathrm{Al}_{2} \mathrm{O}_{3}$. $3 \mathrm{CaSO}_{4} \cdot 31 \mathrm{H}_{2} \mathrm{O}$, or as the monosulfate form, $3 \mathrm{CaO} \cdot \mathrm{Al}_{2} \mathrm{O}_{3} \cdot \mathrm{CaSO}_{4} \cdot 12 \mathrm{H}_{2} \mathrm{O}$, the amounts of lime and tricalcium aluminate required would be as follows:

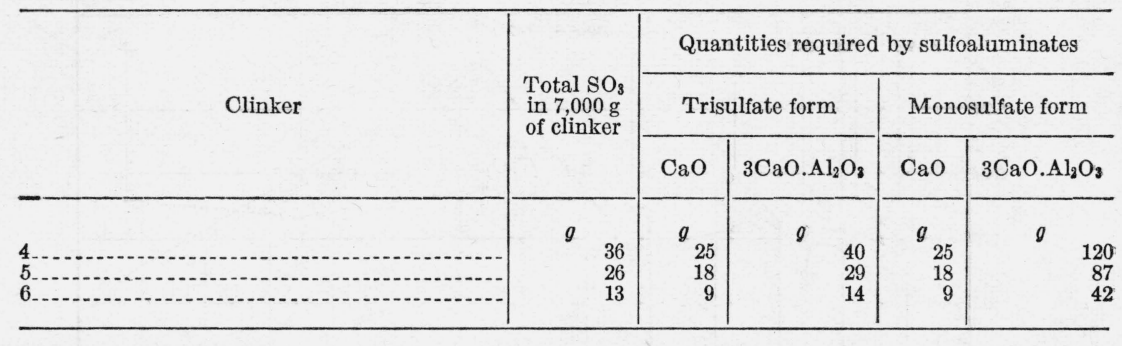

The large increases in the amounts of $\mathrm{R}_{2} \mathrm{O}_{3}$, consisting wholly of alumina, in the 7-minute extracts of clinkers 4,5 , and 6 may therefore be due to the low-lime concentration in these filtrates. This suggestion is supported by the observation that, as the $\mathrm{CaO}$ concentration increased in the interval between 7 minutes and 2 hours, the alumina concentration decreased to values approximating those of untreated filtrates. The alumina was precipitated, presumably, as a calcium aluminate hydrate, although the reactions of the aluminous compounds in the clinker after the sulfate had combined as a sulfoaluminate are not yet definitely known. On the basis of recent results [20], both the monosulfate and trisulfate sulfoaluminates are metastable in the presence of alkali hydroxide $(0.1$ to $0.5 N)$, with respect to members of the solid-solution series,

$$
3 \mathrm{CaO} \cdot \mathrm{Al}_{2} \mathrm{O}_{3} \cdot \mathrm{CaSO}_{4} \cdot 12 \mathrm{H}_{2} \mathrm{O}-3 \mathrm{CaO} \cdot \mathrm{Al}_{2} \mathrm{O}_{3} \cdot \mathrm{Ca}(\mathrm{OH})_{2} \cdot 12 \mathrm{H}_{2} \mathrm{O} \text {. }
$$

Therefore, as the aluminous compounds continue to react, the basicity having increased due to the sulfoaluminate reaction involving potash, a conversion of the metastable products to members of the solid-solution series might be expected. However, this conversion occurs but slowly, and, therefore, calcium aluminate hydrate is probably precipitated as such during the period between 7 minutes and 2 hours considered here.

Figure 11 shows that the changes in the concentration of silica were small except in the extracts from clinkers 1 and 4.

(f) $\mathrm{T} D \mathrm{~A}$

Of the various added materials, T D A (plus 0.23 percent of $\mathrm{H}_{2} \mathrm{O}$ ) resembles gypsum the most closely in its effects on the extracts. For example, comparison of figures 12 and 6 reveals a striking similarity in the graphs showing changes in the concentration of potash.

The decreases in the basicity of the extracts are caused partially by the reduced extractions of the alkalies but perhaps to an even greater extent by inhibition of the sulfoaluminate reaction. This appears to be indicated in figure 12 by increases in the concentration of $\mathrm{SO}_{3}$ in the extracts over those prevailing in the filtrates from pastes without added 


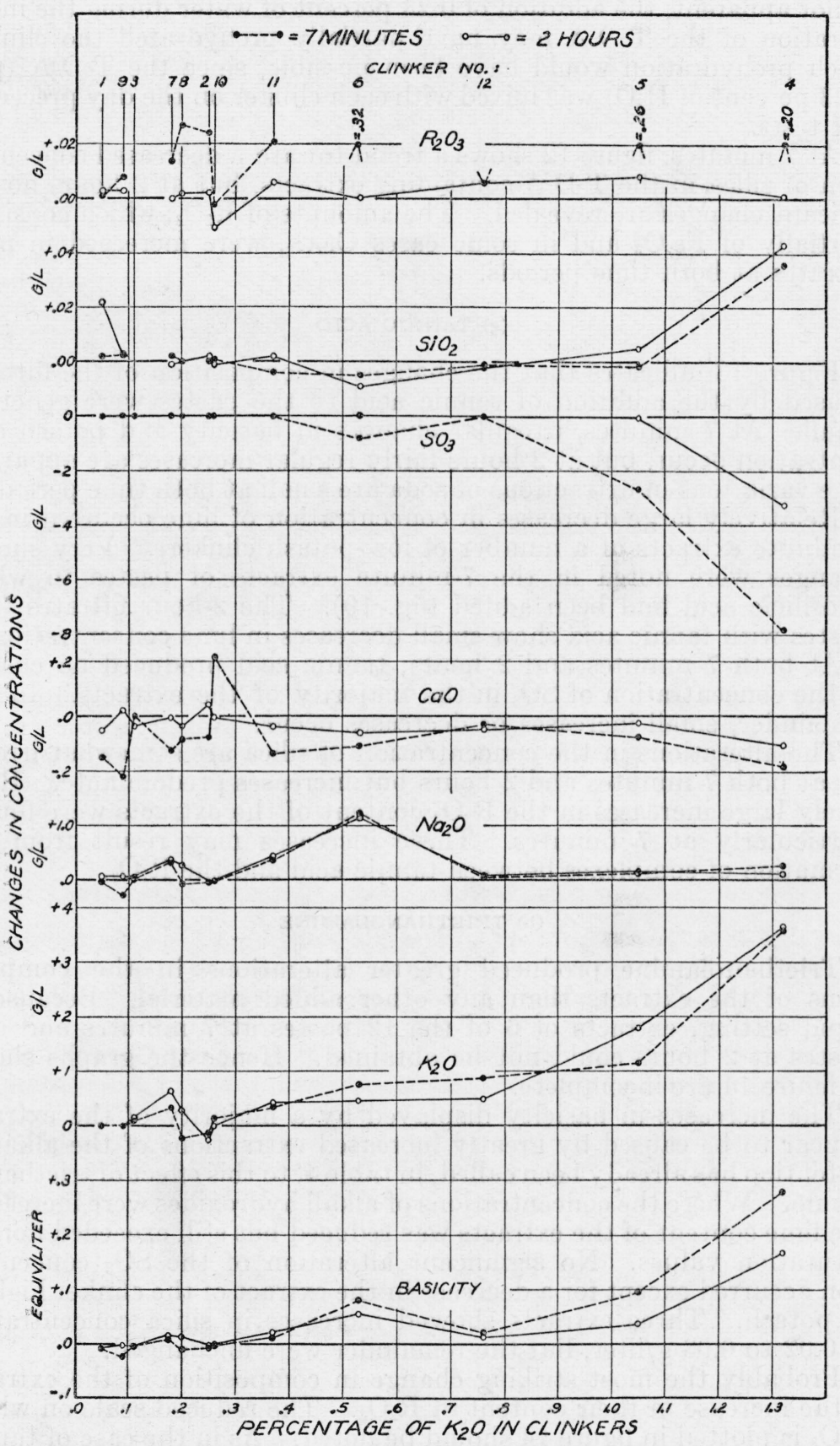

FigURE 11.-Changes in compositions of extracts caused by sucrose. Note variations in scale 
materials. Although the reason for the retardation of this reaction is not apparent, the addition of 0.23 percent of water during the incorporation of the $\mathrm{T}$ ) A may have partially prehydrated the clinker. Such prehydration would have been possible, since the T D A (plus 0.23 percent of $\mathrm{H}_{2} \mathrm{O}$ ) was mixed with each clinker on the day preceding: the tests.

At 7 minutes, figure 12 shows a trend toward a decreased concentration of siliea in the T D A-containing extracts, but at 2 hours no significant changes are revealed. The amounts of $\mathrm{R}_{2} \mathrm{O}_{3}$, which consisted partially of $\mathrm{Fe}_{2} \mathrm{O}_{3}$ and in some cases $\mathrm{Cr}_{2} \mathrm{O}_{3}$, were increased in most filtrates at both time periods.

\section{(g) TANNIC ACID}

Figure 13 indicates that the changes in composition of the filtrates caused by the addition of tannic acid to the pastes were generally small. At 7 minutes, irregular changes in basicity and potash concentration occur, but at 2 hours fairly regular increases are apparent. The variations in extractions of soda are small at both time periods.

Relatively large decreases in concentration of lime occurred in the 7-minute extracts of a number of low-potash clinkers. Very similar changes were noted in the 7-minute extracts of pastes to which fluosilicic acid had been added (fig. 10). The 2-hour filtrates from pastes with tannic acid show small decreases in lime concentration.

At both 7 minutes and 2 hours, tannic acid produced no change in the concentration of $\mathrm{SO}_{3}$ in the majority of the extracts. In the remainder, small increases or decreases occur.

The alterations in the concentration of silica are somewhat irregular at both 7 minutes and 2 hours but increases predominate. Relatively large increases in the $\mathrm{R}_{2} \mathrm{O}_{3}$ content of the extracts were found, particularly at 7 minutes. These increases may result from the formation of complexes between tannic acid and the $\mathrm{R}_{2} \mathrm{O}_{3}$.

\section{(h) TRIETHANOLAMINE}

Triethanolamine produced greater alterations in the compositions of the extracts than any other added material. Because of rapid setting, extracts of 6 of the 12 pastes at 7 minutes and of 2 pastes at 2 hours could not be obtained. Hence the graphs shown in figure 14 are incomplete.

The increases in basicity displayed by a majority of the extracts appear to be caused by greatly increased extractions of the alkalies. Attention has already been called, in table 4, to this effect of triethanolamine. Where the concentrations of alkali hydroxides were increased, the lime content of the extracts was reduced but still exceeded normal saturation values. No significant alteration of the $\mathrm{SO}_{3}$ concentration occurred except for a decrease in the extract of the clinker highest in potash. Three extracts showed increases in silica concentration of 0.02 to $0.03 \mathrm{~g} /$ /iter, but the remainder were unchanged.

Probably the most striking change in composition of the extracts is the increase in their content of $\mathrm{R}_{2} \mathrm{O}_{3}$. The reduced scale on which $\mathrm{R}_{2} \mathrm{O}_{3}$ is plotted in figure 14 should be noted. As in the case of tannic acid, the increases in concentration of $\mathrm{R}_{2} \mathrm{O}_{3}$ may result from the formation of complexes between triethanolamine and ferric and aluminum hydroxides. 


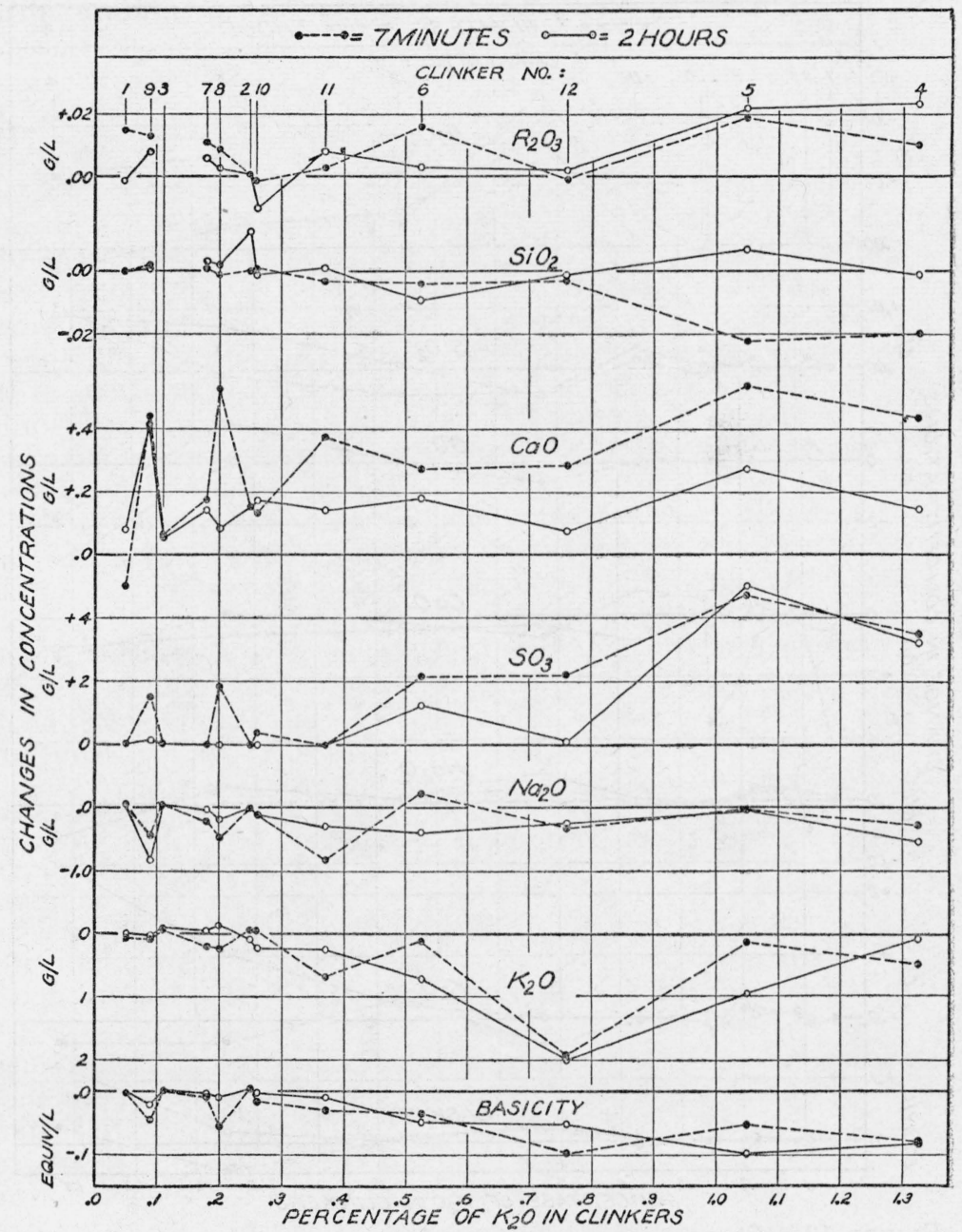

Figure 12.-Changes in compositions of extracts caused by $T D A$. Note variations in scale. 


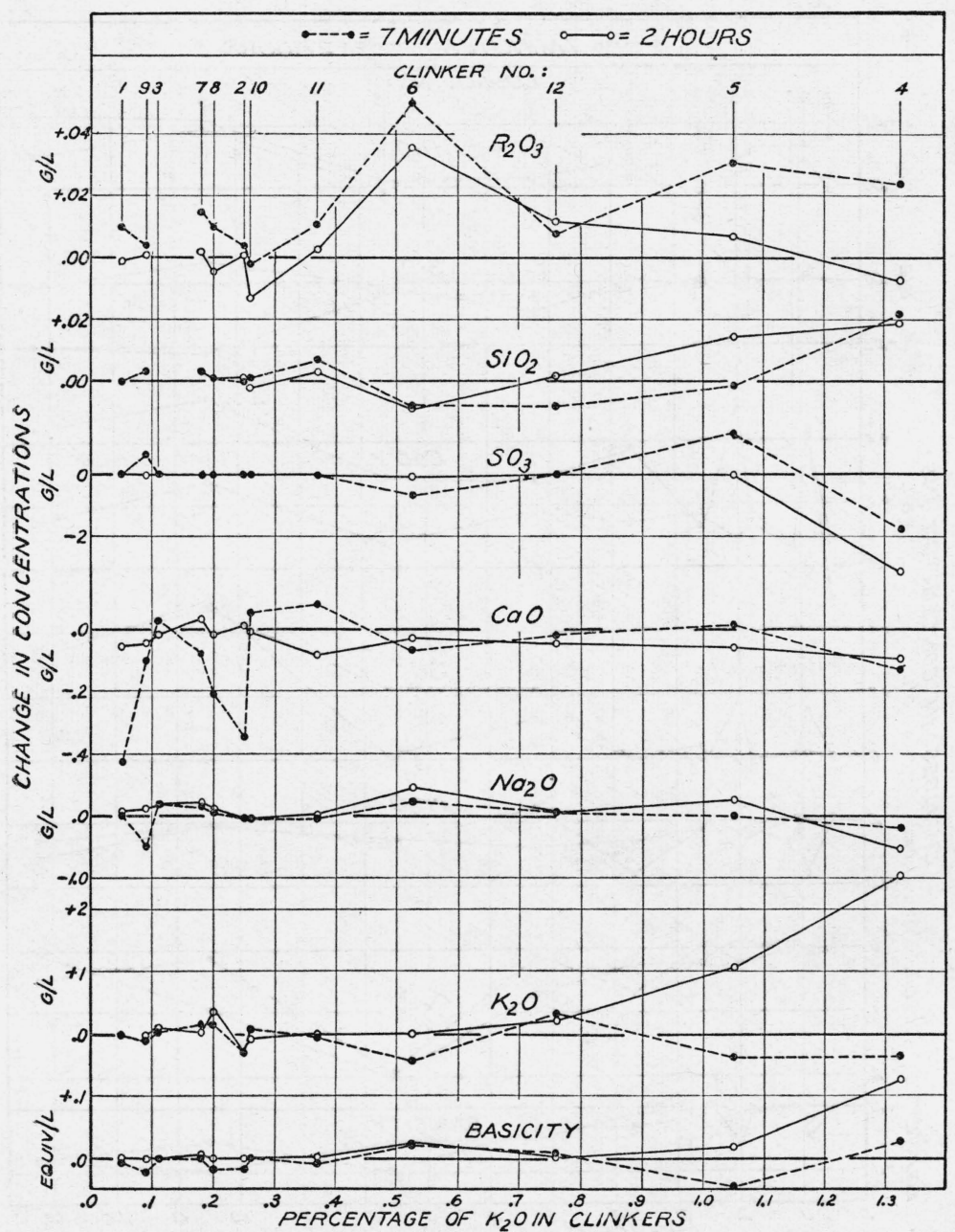

Figure 13.-Changes in compositions of extracts caused by tannic acid. Note variations in scale. 


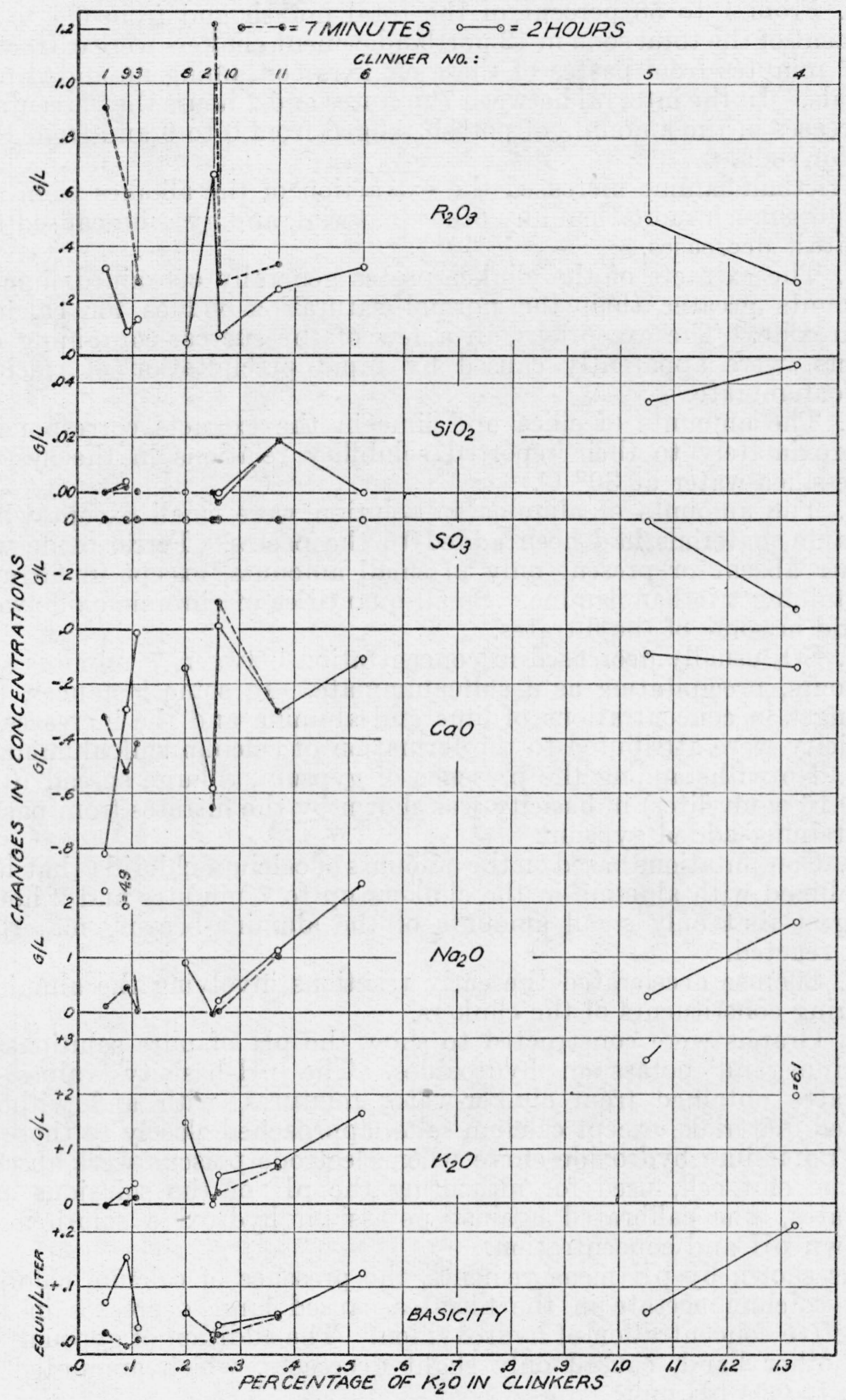

FIGURE 14.-Changes in composition of extracts caused by triethanolamine. Note variations in scale. 


\section{SUMMARY}

1. From 1 to 58 percent of the total potash and from 0.4 to 30 percent of the total soda in 12 portland cement clinkers were extracted in 7 minutes from pastes of these clinkers containing no added materials. In the interval between 7 minutes and 2 hours the percentage increases in the amounts of potash ranged from 0 to 9 and from 1 to 12 for soda.

Triethanolamine increased the extraction of the alkalies from the clinkers much more than any other material, and gypsum caused the greatest decreases.

2 . The extracts of the clinker pastes generally contained lime in amounts greater than the normal saturation values for calcium hydroxide. The exceptions, in a few of the sucrose-containing extracts, were apparently caused by rapid precipitation of calcium sulfoaluminate.

3 . The amounts of silica and lime in the extracts corresponded approximately to their reported solubility relations in the system lime-silica-water at $30^{\circ} \mathrm{C}$.

4. The amounts of alumina in solution were small except when organic materials had been added to the pastes. Ferric oxide was either absent or present only in small amounts, except in filtrates containing triethanolamine. Small quantities of chromic oxide were found in some of the filtrates.

5. $\mathrm{SO}_{3}$ usually decreased in concentration between 7 minutes and 2 hours, precipitating as a sulfoaluminate. In some instances, the changes in concentrations of lime and alumina and the increases in basicity were attributed to the formation of calcium sulfoaluminate.

6. Notwithstanding the presence of gypsum, a large spread $(0.05$ to 0.16 equiv/liter) in basicity was shown by the filtrates from pastes containing added gypsum.

7. Computations based on the amounts of calcium chloride that had combined with alumina in the clinkers up to 7 minutes and 2 hours suggest that only small amounts of the alumina-bearing materials had reacted.

8. Sucrose accelerated the early reactions involving the aluminabearing constituents of the clinkers.

9. Graphs were constructed to show the $\mathrm{pH}$ of pure solutions of calcium and potassium hydroxides. The $\mathrm{pH}$-basicity values of filtrates obtained from clinker-water mixtures, with and without added materials, except calcium salts, approached closely to those of the potassium hydroxide curve when electrode poisons were absent.

The emf cell, used for measuring the $\mathrm{pH}$ of the solutions and filtrates, was calibrated against potassium hydroxide solutions of known $\mathrm{pH}$ and concentration.

As shown by $\mathrm{pH}$ measurements, the presence of calcium chloride and calcium acetate in the filtrates caused large decreases in the effective concentration of hydroxyl ion. The addition of gypsum, on the other hand, caused only slight decreases, which amounted to 0.02 to $0.04 \mathrm{pH}$ unit.

10. The conductivities, surface tensions, and densities of the filtrates are reported. 
The authors are indebted to P. H. Bates, who outlined and directed the research, to G. R. Shelton and E. P. Flint for aid in preparing the results for publication, and W. J. Hamer for his helpful suggestions and interest in the $\mathrm{pH}$ measurements.

\section{REFERENCES}

[1] G. L. Kalousek, C. H. Jumper, and J. J. Tregoning, Rock Products 44, 52 (1941).

[2] E. S. Newman, R. L. Blaine, C. H. Jumper, and G. L. Kalonsek. (Publication pending.)

[3] R. H. Bogue, Ind. Eng. Chem., Anal. Ed. 1, 192 (1929).

[4] L. A. Wagner, Proc. Am. Soc. Testing, Materials 33, pt. 2, 553 (1933).

[5] Scott's Standard Methods of Chemical Analysis (D. Van Nostrand Co. Ine., New York, N. Y., 1935).

[6] J. I. Hoffman and G. E. F. Lundell, J. Research NBS 20, 607 (1938) RP1095.

[7] L. S. Wells, BS J. Research 1, 951 (1928) RP34.

[8] Stahler's Handbuch der Arbeitsmethoden in der anorganischen Chemie (Leipzig) 3, 863 (1914).

[9] W. J. Hamer, Private communication.

[10] H. S. Harned and M. A. Cook, J. Am. Chem. Soc. 59, 496 (1937).

[11] H. S. Harned and W. J. Hamer, J. Am. Chem. Soc. 55, 2194 (1933.)

[12] Int. Crit. Tables 4 (1928).

[13] W. C. Taylor, J. Research NBS 2\%, 311 (1941) RP1421.

[14] W. C. Taylor, J. Research NBS 29, 437 (1942) RP1512.

[15] L. Forsen, Symposium on the Chemistry of Cements (Stockholm) p. 298 (1938).

[16] E. P. Flint and L. S. Wells, BS J. Research 12, 751 (1934) RP687.

[17] G. E. Bessey, Symposium on the Chemistry of Cements (Stockholm) p. 178 (1938).

[18] F. M. Lea and G. E. Bessey, J. Chem. Soc. (London) 1612 (1937).

[19] J. Foret, Compounds of Calcium Salts and Calcium Aluminates (Hermann \& Cie, Paris 1935). F. E. Jones, Symposium on the Chemistry of Cements (Stockholm) p. 231 (1938).

[20] G. L. Kalousek, Sulfoaluminates of calcium as stable and metastable phases, and a study of a portion of the 5 component system $\mathrm{CaO}-\mathrm{Al}_{2} \mathrm{O}_{3}-\mathrm{SO}_{3}-\mathrm{Na}_{2} \mathrm{O}$ $\mathrm{H}_{2} \mathrm{O}$ at $25^{\circ} \mathrm{C}$. (University of Maryland, College Park, Md. Dissertation, June 1941).

Washington, October 14, 1942. 


\section{NATIONAL BUREAU OF STANDARDS,}

WASHINGTON, D. C.

Send me the Mathematical Tables marked $\mathrm{X}$ below. I enclose remittance ${ }^{1}$ to cover the cost.

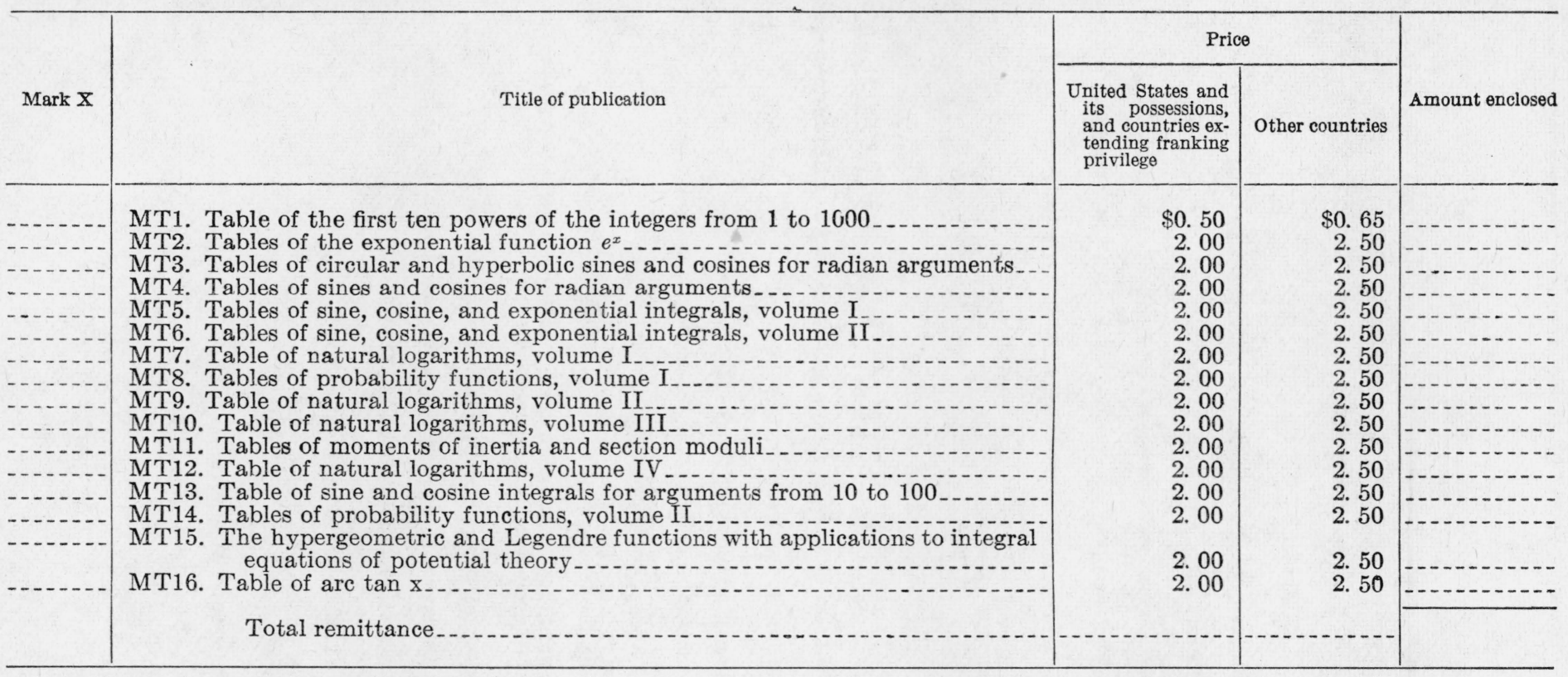

${ }^{1}$ Remittance should be in form of post-office money order, or check, and made payable to the order of the "National Bureau of Standards" in United States currency.

Send to

Number and Street

City and State 


\section{MATHEMATICAL TABLES}

Attention is invited to a series of publications which is being prepared by the Project for the Computation of Mathematical Tables conducted by the Federal Works Agency, Work Projects Administration for the City of New York under the sponsorship of the National Bureau of Standards. The tables which have been made available through the National Bureau of Standards are listed below.

There is included in this list a publication on the hypergeometric and Legendre functions (MT15), prepared by the Bureau.

MT1. Tablz of the First Ten Powers of the Integrrs From 1 to 1000:

(1938) VIII +80 pages; heavy paper cover. 50 cents.

MT2. Tables of the Exponential Function $e^{x}$.

The ranges and intervals of the argument and the number of decimal places in the entries are given below:

$\begin{array}{lcc}\text { Range of } x & \text { Interval of } x & \text { Decimal given } \\ -2.5000 \text { to } 1.0000 & 0.0001 & 18 \\ 1.0000 \text { to } 2.5000 & .0001 & 15 \\ 2.500 \text { to } 5.000 & .001 & 15 \\ 5.00 \text { to } 10.00 & .01 & 12\end{array}$

(1939) $\mathrm{XV}+535$ pages; bound in buckram, $\$ 2.00$.

MT3. Tables of Circular and Hyperbolic Sines and Cosines for Radian Arguments:

Contains 9 decimal place values of $\sin x, \cos x, \sinh x$ and $\cosh x$ for $x$ (in radians) ranging from 0 to 2 at intervals of 0.0001 .

(1939) XVII + 405 pages; bound in buckram, $\$ 2.00$.

MT4. Tables of Sines and Cosines for Radian Arguments:

Contains 8 decimal place values of sines and cosines for radian arguments ranging from 0 to 25 at intervals of 0.001 .

(1940) XXIX + 275 pages; bound in buckram, $\$ 2.00$.

MT5. Tables of Sine, Cosine, and Exponential Integrals, Volume I:

Values of these functions to 9 places of decimals from 0 to 2 at intervals of 0.0001 .

(1940) XXVI + 444 pages; bound in buckram, $\$ 2.00$.

MT6. Tables or Sine, Cosine, and Exponential Integrals, Volume II:

Values of these functions to 9,10 , or 11 significant figures from 0 to 10 at intervals of 0.001 , with auxiliary tables.

(1940) XXXVII + 225 pages; bound in buckram, $\$ 2.00$.

MT7. Table of Natural Logarithms, Volume I:

Logarithms of the integers from 1 to 50,000 to 16 places of decimals.

(1941) XVIII + 501 pages; bound in buckram, $\$ 2.00$.

MT8. Tables of Probability Functions, Volume I:

Values of these functions to 15 places of decimals from 0 to 1 at intervals of 0.0001 and from 1 to 5.6 at intervals of 0.001 .

(1941) XXVIII + 302 pages; bound in buckram, $\$ 2.00$.

[Continued on p. 4 of cover] 
MT9. Table or Natural Logarithms, Volume II:

Logarithms of the integers from 50,000 to 100,000 to 16 places of decimals.

(1941) XVIII+ 501 pages; bound in buckram, $\$ 2.00$.

MT10. Table of Natural Logarithms, Volume III:

Logarithms of the decimal numbers from 0.0001 to 5.0000 , to 16 places of decimals.

(1941) XVIII+501 pages; bound in buckram, $\$ 2.00$.

MT11. Tables of the Moments of Inertia and Section Moduli of Ordinary Angles, Chan. nels, and Bulb Angles with Certain Plate Combinations:

(1941) XIII +197 pages; bound in green cloth. $\$ 2.00$.

MT12. Table of Natural Logarithms, Volume IV:

Logarithms of the decimal numbers from 5.0000 to 10.0000 , to 16 places of decimals.

(1941) XXII + 506 pages; bound in buckram, $\$ 2.00$.

MT13. Table or Sine and Cosine Integrals por Arguments From 10 to 100:

(1942) XXXII+185 pages, bound in buckram, $\$ 2.00$.

MT14. Tables of Probability Functions, Volume II:

Values of these functions to 15 places of decimals from 0 to 1 at intervals of 0.0001 and from 1 to 7.8 at intervals of 0.001 .

(1942) XXI + 344 pages; bound in buckram, $\$ 2.00$.

MT15. The hypergeometric and Legendre functions with applications to integral equations of potential theory. By Chester Snow, National Bureau of Standards. Reproduced from original handwritten manuscript.

(1942) VII + 319 pages; bound in heavy paper cover. $\$ 2.00$.

MT16. TABLb of ARc Tan X:

Table of inverse tangents for positive values of the angle in radians. Second central differences are included for all entries.

$\begin{array}{rrr}\text { Range of } x & \begin{array}{c}\text { Interval between } \\ \text { successive arguments }\end{array} \\ 0 \text { to } & 7 & 0.001 \\ 7 \text { to } & 50 & .01 \\ 50 \text { to } & 300 & .1 \\ 300 \text { to } 2,000 & 1 \\ 2,000 \text { to } 10,000 & 10\end{array}$

(1942) XXV +169 pages; bound in buckram, $\$ 2.00$.

Payment is required in advance. Make remittance payable to the "National Bureau of Standards," and send with order, using the blank form facing page 3 of the cover.

The prices are for delivery in the United States and its possessions and in countries extending the franking privilege. To other countries the price of $\mathrm{MT} 1$ is 65 cents; that of MT2 to MT16, inclusive, is $\$ 2.50$ each; remittance to be made payable in United States currency.

Copies of these publications have been sent to various Government depositories throughout the country, such as public libraries in large cities, and colleges and universities, where they may be consulted.

A mailing list is maintained for those who desire to receive announcements regarding new tables as they become available. 